\title{
Parenting Stress as A Moderator of the Relationship Between Child Emotional and Behavioral Problems and Parental Readiness for Change
}

\section{Connor Fais}

West Virginia University, crfais@mix.wvu.edu

Follow this and additional works at: https://researchrepository.wvu.edu/etd

Part of the Counseling Psychology Commons

\section{Recommended Citation \\ Fais, Connor, "Parenting Stress as A Moderator of the Relationship Between Child Emotional and Behavioral Problems and Parental Readiness for Change" (2021). Graduate Theses, Dissertations, and Problem Reports. 10330. \\ https://researchrepository.wvu.edu/etd/10330}

This Dissertation is protected by copyright and/or related rights. It has been brought to you by the The Research Repository @ WVU with permission from the rights-holder(s). You are free to use this Dissertation in any way that is permitted by the copyright and related rights legislation that applies to your use. For other uses you must obtain permission from the rights-holder(s) directly, unless additional rights are indicated by a Creative Commons license in the record and/ or on the work itself. This Dissertation has been accepted for inclusion in WVU Graduate Theses, Dissertations, and Problem Reports collection by an authorized administrator of The Research Repository @ WVU. For more information, please contact researchrepository@mail.wvu.edu. 
Parenting Stress as A Moderator of the Relationship Between Child Emotional and Behavioral Problems and Parental Readiness for Change

Connor Fais 
Parenting Stress as A Moderator of the Relationship Between Child Emotional and Behavioral Problems and Parental Readiness for Change

Connor Fais, M.A.

\author{
Dissertation submitted \\ to the College of Education and Human Services \\ at West Virginia University \\ in partial fulfillment of the requirements for the degree of \\ Doctor of Philosophy in \\ Counseling Psychology
}

\author{
Monica Leppma, Ph.D., Chair \\ Laura Capage, Ph.D. \\ Jeffrey Daniels, Ph.D. \\ Lisa Platt, Ph.D. \\ Department of Counseling and Learning Sciences
}

Morgantown, West Virginia

2021

Keywords: Parenting stress, internalizing problems, externalizing problems, parental readiness for change

Copyright 2021 Connor Fais, M.A. 


\section{ABSTRACT \\ Parenting Stress as A Moderator of the Relationship Between Child Emotional and Behavioral Problems and Parental Readiness for Change}

\section{Connor Fais}

The current study applied the Transtheoretical Model of Behavior Change (Prochaska \& DiClemente, 1983) to parenting behavior, as past research has suggested that increasing parental motivation and readiness for change is associated with greater client participation and improved therapeutic outcomes (Dowell \& Ogles, 2010; Karver et al., 2006). The current study attempted to replicate Jones et al.'s (2017) primary findings and add to the current body of literature on parental readiness for change by examining parenting stress as a moderator of the relationship between internalizing and externalizing child problems and parental readiness for change (Jones et al., 2017; Wade \& Andrade, 2015). Data was collected at a medium-sized outpatient child, adolescent, and family therapy clinic in the Appalachian region. Guardians completed the Behavior Assessment System for Children, Third Edition (BASC-3), Parenting Readiness for Change Scale (PRFCS), Parenting Stress Index Fourth Edition (PSI-4)/Parenting Stress Index, Fourth Edition Short-Form (PSI-4-SF) or the Stress Index for Parents of Adolescents (SIPA; Sheras et al., 1998). The moderator was not examined due to the violation of multicollinearity and an insufficient number of participants in the high-stress parenting group. The current study found that after controlling for child age and family court status, child internalizing problems, externalizing problems, and parenting stress did not predict level of parental readiness for change. An exploratory analysis found that externalizing problems predicted parenting stress over and above just internalizing problems. 


\section{DEDICATION}

To all those who strive to become the best version of themselves. 


\section{ACKNOWLEDGEMENTS}

I would like to acknowledge and give special thanks to Dr. Laura Capage, Melisa Efe, and Lindsay Druskin. Without these individuals, this project would not have been possible. I would also like to thank my dissertation chair, Dr. Monica Leppma, and my dissertation committee members, Dr. Jeffrey Daniels, and Dr. Lisa Platt, for helping me reach this final moment in my journey to become a psychologist. Additionally, I would like to extend thanks to Dr. Meghan Brown and Dr. Jennifer Brody for their guidance and supervision over the past year of doctoral internship with Astor Services for Children and Families. I am excited to also provide a huge thank my lovely partner, Kristine Chen, who has provided unyielding faith and encouragement and who has been there for me through all the most difficult patches along the way. I would like to thank my parents, Beth and Robert Fais, and my brother Collier, for their unconditional love and support - I would not have been able to do this without all of you. 


\section{TABLE OF CONTENTS}

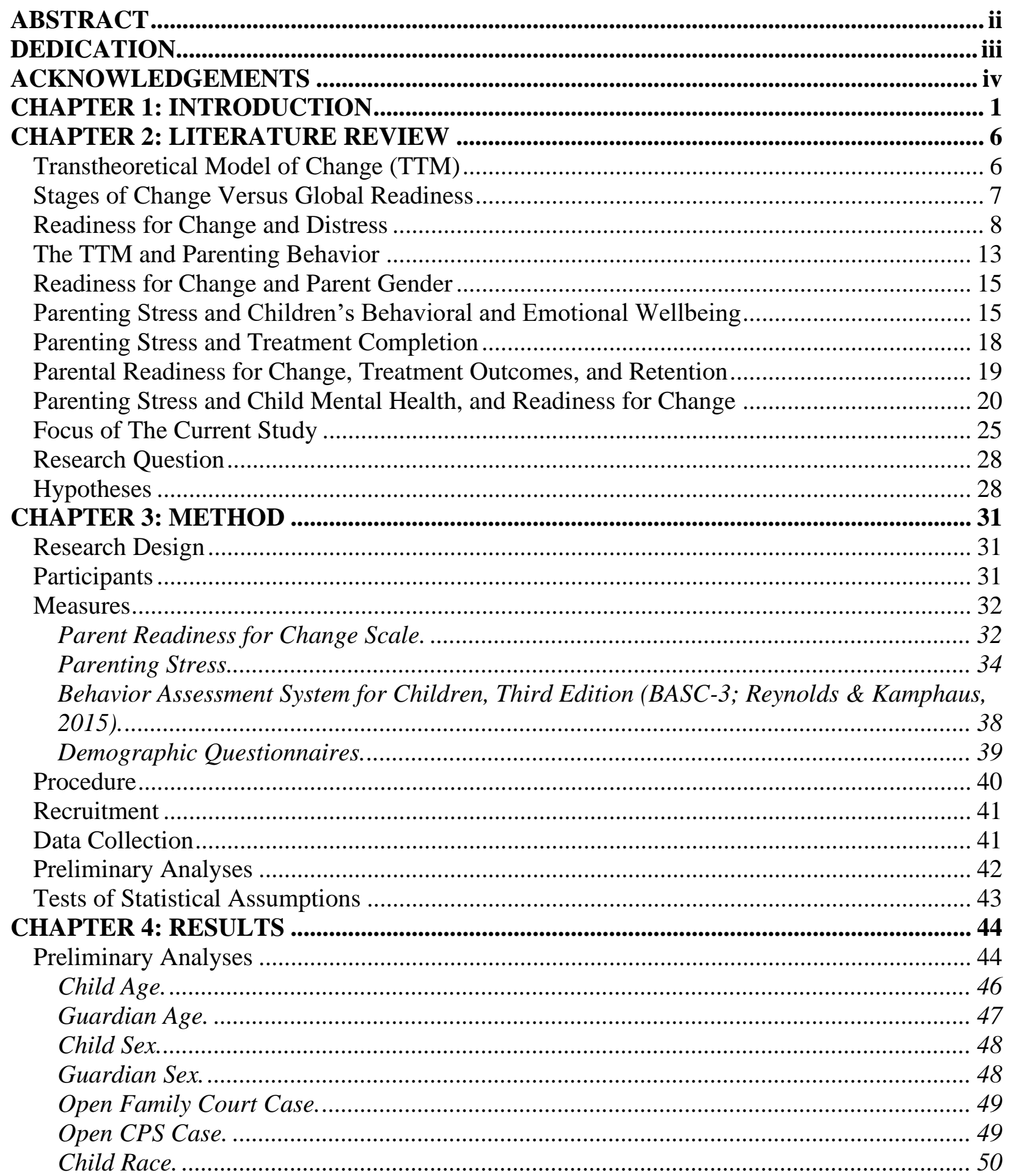




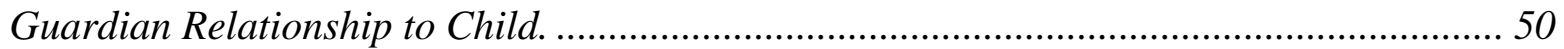

Guardian Years of Formal Education........................................................................ 51

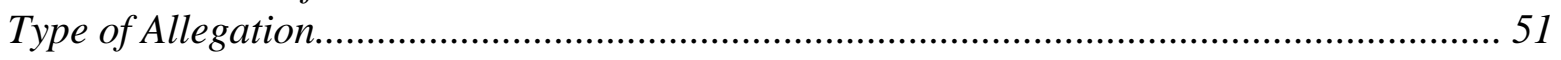

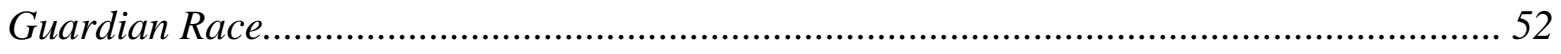

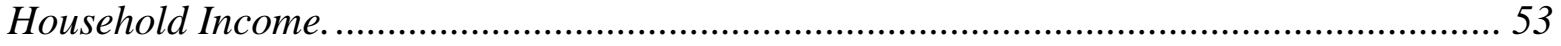

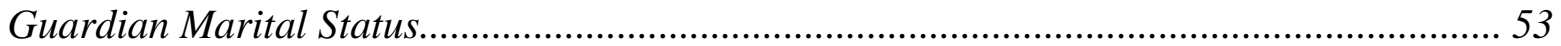

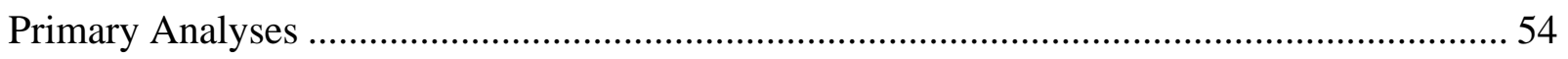

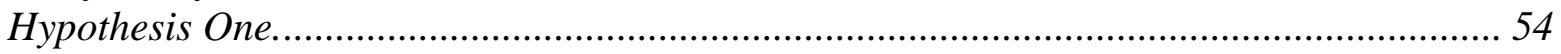

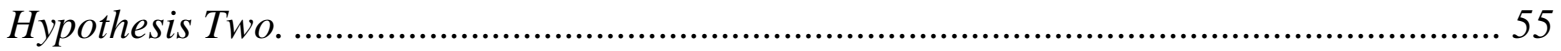

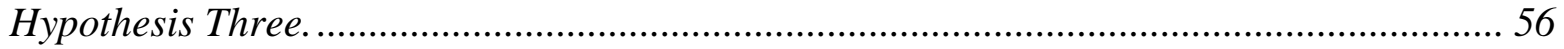

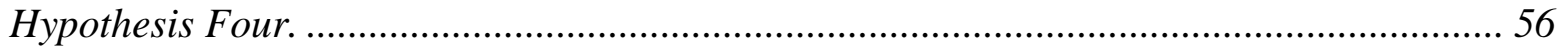

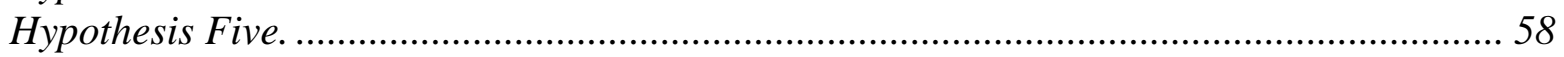

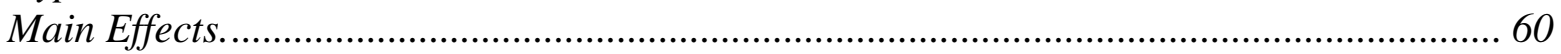

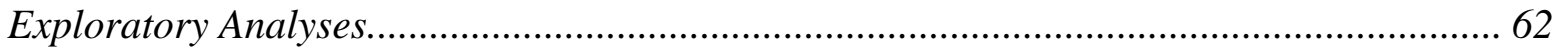

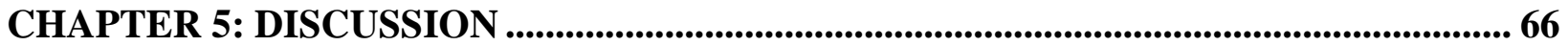

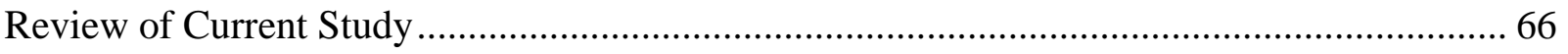

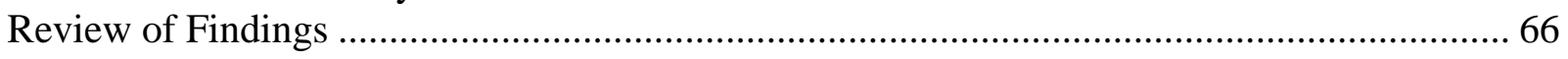

Readiness for Change and Demographic Variables. .................................................. 66

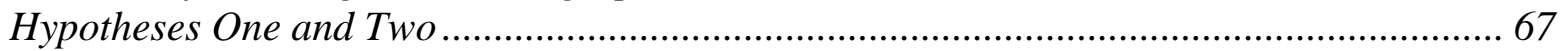

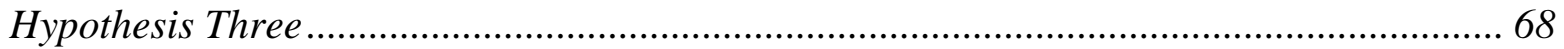

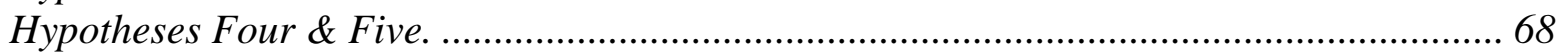

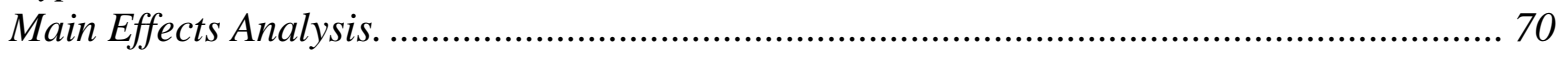

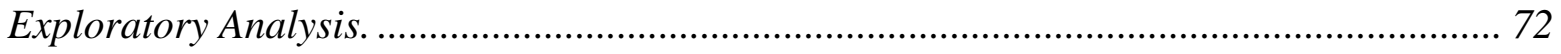

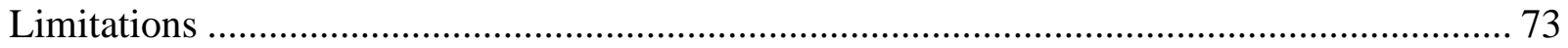

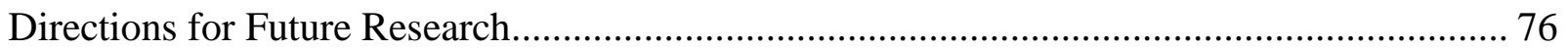

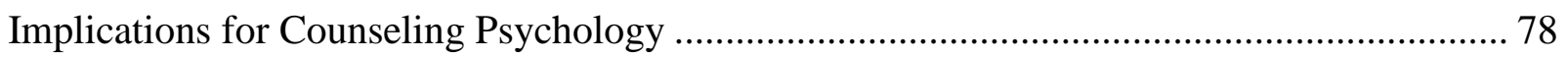

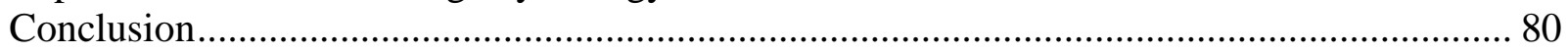

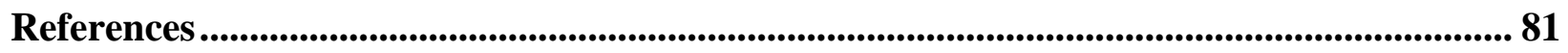

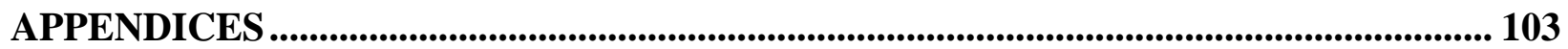

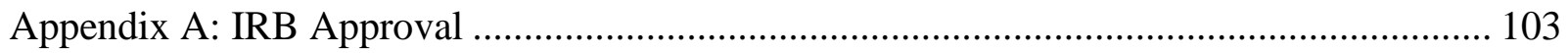

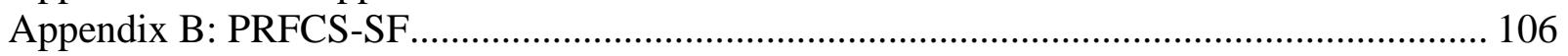

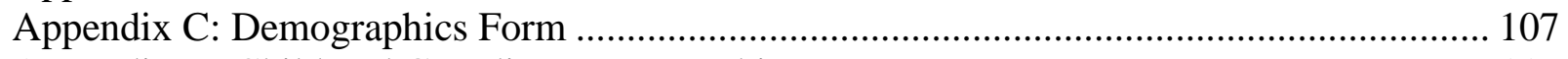

Appendix D: Child and Guardian Demographics .......................................................... 117

Appendix E: Means, standard deviations, and bivariate correlations among Parental Readiness for Change, Parenting stress, Internalizing Problems, and Externalizing Problems .............. 119 Appendix F: Multiple Regression Predicting Parenting Stress From Child Age, Family Court Case Status, Internalizing problems, Externalizing problems, and Parenting Stress ............. 120 Appendix G: Hierarchical Multiple Regression Predicting Parenting Stress From Internalizing

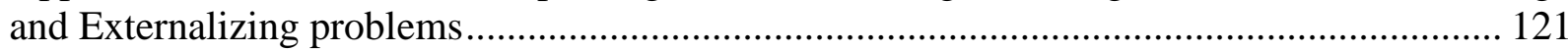

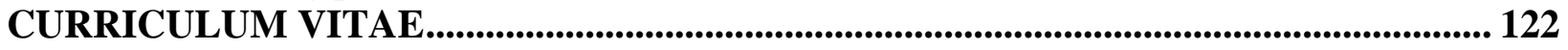




\section{CHAPTER 1: INTRODUCTION}

Research as shown that higher levels of parental readiness for change are associated with increased parent participation in their child's treatment (Wade \& Andrade, 2015). The current study examines parental readiness for change to better help researchers and clinicians understand the relationship between parental readiness for change, parenting stress, and child behavioral/emotional problems. Several negative long-term outcomes are associated with internalizing and externalizing child behavior problems in children and adolescents. For instance, adolescent anxiety disorders have been found to be associated with a greater likelihood of drug use problems (e.g., nicotine and alcohol use), depression, educational problems, increased suicide risk, and anxiety problems later in life (Woodward \& Fergusson, 2001). Furthermore, childhood depression appears to increase the risk of poorer adaptive functioning and aggression later in life (Aronen, \& Soininen, 2000). Additionally, there is some longitudinal evidence that internalizing and externalizing problems are reciprocal, and that externalizing problems sustain subsequent internalizing problems over time (Stone et al., 2015). Also, children who develop and sustain higher levels of externalizing behaviors between grades two and eight show a greater risk of violence and delinquency (e.g., stealing, breaking and entering; Harachi et al., 2006).

Research on parental readiness for change is relatively new. For instance, Nock and Ferriter (2005) stated, “...we could not find a single study that systematically measured and evaluated the role of parent motivation for child therapy_-suggesting this is an area in great need of attention” (Nock \& Ferriter, 2005, p. 155). Further, Littell and Girvin’s (2005) stated, “There is little empirical research on readiness for change among caregivers of abused and neglected 
children" (p. 61). Similarly, Jones et al. (2017) acknowledged that parental readiness for change and childhood behavior problems are not well represented in existing literature. Therefore, this study may serve as a primary step in understanding readiness for change and its relationship to clinical variables (e.g., child emotional/behavioral problems). In a preliminary study, Jones and colleagues (2017) used the Transtheoretical Model of Behavior Change (TTM; Prochaska \& DiClemente, 1983) to better understand the parents' motivation to participate in their child's therapeutic interventions. They noted that their interest in parent motivation was in part due to common role that parent's play in their child's own mental health treatment services (e.g., implementing behavioral reinforcement strategies in the home). Furthermore, only a few studies have examined these variables, which have examined parental readiness for change and its relationship with child behavior problems (e.g., Andrade et al., 2015; Proctor et al., 2017).

Parental involvement is a key aspect related to positive therapeutic outcomes for children (Dowell \& Ogles, 2010; Karver et al., 2006). For example, when psychoeducation on the purpose of therapy and child symptoms was implemented with parents, they showed greater levels of treatment engagement (Martinez et al., 2017). Similarly, a meta-analysis of 41 studies found that including parents in children's psychotherapy treatment had a medium effect $(d=$ 0.27) over and above child-only interventions (Dowell \& Ogles, 2010). However, for the treatment of anxiety disorders, parent involvement did not appear to improve therapy outcomes (Thulin et al., 2014). However, additional research indicated that parental participation in their child's treatment is beneficial. Specifically, Karveret al. (2006) analyzed 49 clinical studies and found that the unweighted effect size of parental participation on treatment outcomes was .26. These researchers noted, “... without parental involvement, it may be less likely that therapeutic 
changes implemented with a child client will be generalized to the home setting" (Karver et al., 2005, p. 44), indicating that parental engagement in their child's therapy plays an important role in promoting successful treatment outcomes.

Researchers have worked to identify ways to increase engagement with treatment (e.g., Rutkowski et al., 2010; Sirey, 2013; Torres, et al., 2019) and develop interventions that may increase client motivation (Nock \& Kazdin, 2005). Also, treatment adherence been linked to more successful treatment outcomes for parents and children (Kazdin et al.,1994). Findings have indicated that significant improvements in follow-up parental readiness for change was associated with decreased therapeutic attrition rates (Webb et al., 2017). Research has also shown that mothers of children who successfully participate in treatment reported greater longterm outcomes, including reduced parenting stress, less child behavior problems, and a decreased likelihood that parents perceive their child's behavior as problematic (Boggs et al., 2004).

Haine-Schlagel and Walsh (2015) have called for additional efforts to identify ways to improve parental participation engagement (e.g., Chacko et al., 2012). Haine-Schlagel and Walsh (2015) found that parental participation engagement in their child's therapeutic process (Karver et al., 2005; King et al., 2014) is associated with increased session attendance. Adequate participation and retention in therapy are significant considering that approximately $36-44 \%$ of children and adolescents drop out or do not complete therapy (de Haan et al., 2013). Furthermore, approximately $28 \%$ of families attending therapy attend two or fewer sessions (Masi et al., 2003). Some evidence has shown that for adults, motivational enhancement (e.g., role induction and motivational interviewing) may be helpful for increasing participation in therapy (see Walitzer et al., 1999, for a review). For instance, role induction may involve 
providing the client with an understanding of what to expect from therapy and describe activities involved in a typical session (Delgadillo \& Groom, 2017; Macaskill, 1989; Orne \& Wender, 1968). Motivational interviewing (Miller \& Rollnick, 2002, 2013) may also help clients reduce ambivalence towards making changes. For instance, Torres and colleagues (2019) found that motivational interviewing was associated with increased rehabilitation services engagement and a stronger perceived therapeutic alliance.

Readiness for change may serve as a key to increasing parent participation (Sterrett et al., 2010). Furthermore, parental participation engagement appears to be related to readiness for change and additional research on parental readiness for change may help develop a better understanding of its potential relationship with increased treatment engagement (Becker et al., 2015; Brestan et al., 1999; Chaffin et al., 2009; DiClemente, \& Prochaska, 1982; Haine-Schlagel, \& Walsh, 2015; Miller \& Rollnick, 2002, 2013; Nock \& Ferriter, 2005; Prochaska, \& DiClemente, 1982, 1983; Prochaska et al., 1985; Staudt, 2007; Wade \& Andrade, 2015). The current study may help clinicians develop targeted interventions to increase parent motivation for treatment (Chaffin et al., 2009; Wade, \& Andrade, 2015; Webb et al., 2017).

Jones and colleagues (2017) found that parenting stress was positively related to readiness for change, and that readiness for change was positively associated with child externalizing behaviors. Jones et al.'s (2017) findings support the "threshold" model of parental readiness for change, suggesting there is a "...level of parenting stress at which it no longer matters whether the child has behavior problems and the parent is ready to change regardless of child behavior..." (p. 227). Specifically, they found that when parenting stress was high, parental readiness for change was also high at both low and high levels of child externalizing behavior 
problems. The results from Jones et al. (2017) described this model, suggesting that parenting stress lowers a "threshold" by which parents report their readiness for change. The current study will help support the "threshold" model for both internalizing and externalizing problems and help clarify whether the model also applies to an underserved population commonly seeking services for trauma and abuse (e.g., Office for Victims of Crime, 2017; Wherry et al., 2015; Wolfteich \& Loggins, 2007). The current study will attempt to replicate Jones et al.'s (2017) primary findings and add to the current body of literature on this topic by examining parenting stress as a moderator of the relationship between internalizing and externalizing child problems and again show that parental stress is associated with increased readiness for change among parents (Jones et al., 2017; Wade \& Andrade, 2015). 


\section{CHAPTER 2: LITERATURE REVIEW}

\section{Transtheoretical Model of Change (TTM)}

The transtheoretical model of behavior change (TTM; DiClemente \& Prochaska, 1982; Prochaska, \& DiClemente, 1982, 1983; Prochaska et al., 1985) was originally developed out of research that examined the process of smoking cessation (DiClemente \& Prochaska, 1982; DiClemente et al., 1985; Prochaska, \& DiClemente, 1983) and has been used as an overarching model to explain the psychotherapeutic change process (Prochaska, 1979; Prochaska, \& DiClemente, 1982). DiClemente and Prochaska (1982) took some of the early steps in identifying differences among change process themes between 'self-quitters,' or individuals that had achieved smoking cessation without intervention and individuals who participated in a cessation program. These researchers found that self-directed cessation was not specific to tobacco use history or severity and showed that some individuals were able to initiate 'selfchange' without a program intervention. DiClemente and Prochaska (1982) commented on the interesting nature of self-initiated smoking cessation and reflected on working to further understanding how the self-change process occurs.

A year later, Prochaska and DiClemente (1983) published another study that continued to examine the change process themes. This study classified participants into five stages of change (i.e., precontemplation, contemplation, action, maintenance, and relapse). These groups included individuals that had no plan to quit smoking (pre-contemplative stage), individuals who reported that they had plans to quit smoking (contemplative stage), individuals that had quit smoking as recently as six months ago (action stage), individuals who had quit smoking for six months or more (maintenance stage), and individuals who had recently experienced a smoking relapse 
(relapse stage). These five stages of change have often been used to conceptualize the change process (McConnaughy et al., 1983; Prochaska, \& DiClemente, 1983; Prochaska et al., 1994).

Variations to the original 5-stage model have also been developed. For instance, The University of Rhode Island Change Assessment Questionnaire (URICA), developed by McConnaughy and colleagues (1983), measures participants' readiness for change in psychotherapy along four stages which included pre-contemplation, contemplation, action, and maintenance. Example questions and their corresponding subscale may serve as good descriptors of each stage of change: “As far as I'm concerned, I don't have any problems that need changing” (Precontemplation), "I have a problem, and I really think I should work on it" (Contemplation), "I am really working hard to change" (Action), and "I have been successful in working on my problem, but I'm not sure I can keep up the effort on my own" (Maintenance; McConnaughy, et al., 1983).

\section{Stages of Change Versus Global Readiness}

Other models, including Willoughby and Edens' (1996) two-factor solution, diverge from DiClemente and Hughes' (1990) earlier findings that suggested the existence of a five-factor solution (i.e., Precontemplation, Ambivalent, Participation, Uninvolved or Discouraged, and Contemplation clusters). Mixed findings regarding the factor structure have been pointed out by Carey and colleagues (1999). Moreover, Carey et al.'s (1990) review of the literature suggested that previous research has identified between two and nine readiness for change factors. Carey et al. (1990) suggested that these findings appear to contradict the underlying implications of McConnaughy et al.’s (1983) stage-wise model of change readiness. Carey et al. (1990) suggested that the predictive validity of the stages of change appear to be more consistent as a 
combined model, rather than isolated subscales (see also Belding et al., 1997; Henderson et al., 2004). Wade and Andrade (2015) showed that contemplation and action factors were significantly positively related to one another, and that both were significantly negatively related to the precontemplation factor (all $p s<.001$ ). They also showed that parenting readiness for change may be better represented as a continuous readiness factor (Wade \& Andrade, 2015). The Parental Readiness for Change Scale - Short Form (PRFCS-SF) has been used in this way (e.g., Jones et al., 2017). As a result, the current study will use the continuous/global readiness model in the current study.

\section{Readiness for Change and Distress}

The current study will examine the relationship between parenting stress and readiness to change parenting behavior. Evidence suggests that readiness for change may be related to perceived distress. A study by Alexander and Morris (2008) gathered data from a sample of men that were court-ordered to attend an Abuser Intervention Program. This study measured alcohol use, anxiety, depression, change readiness, and interpersonal conflict. Using cluster analysis, the researchers categorized participants into two change readiness groups; one characterized by lower levels of change readiness and the other characterized by higher levels of change readiness. The authors conducted a multivariate analysis of variance (MANOVA) to compare both groups and found that participants in the high change readiness cluster reported significantly higher levels of distress. The researchers also found that these individuals were more likely to report higher levels of depression and anxiety, and more severe anger-related issues. These results suggest that individuals with lower levels of change readiness may be less 
likely to report psychological distress compared to individuals with higher levels of change readiness.

Past research that examined voluntary versus involuntary interventions may help illustrate the connection between change readiness and perceived distress. Specifically, O'Hare (1996a) examined associations between voluntary versus involuntary (i.e., court-ordered) interventions, readiness for change and perceived problem severity. O'Hare (1996a) found that individuals who were required to attend therapeutic services reported lower levels of change readiness and lower levels of symptom severity. Additionally, O'Hare (1996a) found that individuals who were required to attend therapy showed significantly lower levels of change readiness.

In a subsequent study, O’Hare (1996b) found that individuals who reported higher levels of change readiness tended to report greater levels of severity for psychophysiological and family pathology symptoms, compared to individuals who reported lower levels of change readiness. According to O'Hare (1996b), “About half of the clients in this sample appeared to be minimizing their difficulties or were, at best, marginally involved in the process of change" ( $p$. 17), and “...clients who are more seriously distressed by psychological or family-related problems are likely to be either seriously considering change or ready to take action" (p. 17). This reflection on the findings appears to be consistent with the TTM and suggests that individuals who were not ready for treatment were more likely to report less distress than those who are ready to address a problem.

There is additional evidence that readiness for change is associated with greater levels of reported psychological distress. Specifically, Smith and Tran (2007) examined levels of 
depression, anxiety, alcohol use, and readiness to change among a sample of college students ( $n$ $=233$ ). Results of separate regression analyses showed that increased levels of both anxiety and depression among students who reported problematic levels of alcohol use predicted increased readiness to change alcohol use behavior. A more recent study by Alley and colleagues (2018) supported these results, also showing that readiness to change was significantly positively associated with problematic alcohol use.

Readiness for change has been applied to a variety of clinical issues, including gambling behavior problems (Petry, 2005), sexual offender treatment (Sowden, \& Olver, 2017), alcohol use problems (Demmel et al., 2004), exercise behavior (Daley \& Duda, 2006), smoking cessation (DiClemente et al., 1991; DiClemente et al., 1985; Prochaska et al., 1988), and domestic violence (Scott \& Wolfe, 2003), along with numerous other clinical applications of behavior change (see Prochaska et al., 1994). This current study will focus specifically on parents' readiness for change to address a parenting-related problem. Readiness in this regard has been examined previously and, in the few primary examples that exist in the literature, readiness for change has been examined in relation to interventions for the treatment of child behavior problems (e.g., Jones et al., 2017; Littell \& Girvin, 2005; Wade \& Andrade, 2015).

A meta-analytic study by Norcross and colleagues (2011) that examined the results of 39 studies found that readiness for change had, on average, a medium $(d=.46)$ effect on treatment outcome. Other research indicated that readiness for change may help explain the relationship between initial levels of perceived symptom severity and treatment outcomes. Particularly, Boswell and colleagues (2012) examined the moderating effect of readiness for change on the relationship between symptom severity and treatment outcome for a sample of 
participants seeking mental health treatment. Correlational analyses indicated that overall readiness prior to treatment (i.e., computed by subtracting pre-contemplative scores from the sum of contemplative, action, and maintenance scores) was significantly positively associated with self-reported levels of anxiety and depression prior to treatment, and significantly associated with levels of anxiety and depression symptom reduction after treatment. Furthermore, Boswell et al. (2012) found that higher levels of perceived symptom severity prior to treatment predicted smaller treatment gains. In other words, participants who reported lower levels of symptom severity prior to treatment tended to report more symptom reduction after receiving services. The researchers examined this relationship further by conducting multiple regression analysis and found that readiness moderated the relationship between reported symptom severity prior to treatment and therapeutic outcomes. More specifically, for participants who reported high levels of change readiness, greater levels of depression and anxiety prior to treatment were associated with greater symptom reduction after treatment, an association that only emerged after the researchers entered readiness for change as a moderator variable (Boswell et al., 2012).

Willoughby and Edens (1996) gathered data from 144 inpatient participants that underwent a substance abuse treatment program in Veterans Affairs (VA) Domiciliary care. Hierarchical cluster analysis supported the two-factor solution and revealed significant differences between precontemplation and contemplation/action groups. Specifically, participants categorized into the contemplation/action cluster were found to endorse greater openness to help, indicate greater help seeking behavior prior to the study, report higher levels of guilt and worry about their alcohol use, and display a greater understanding of the pros of their 
alcohol use (i.e., social functioning and mood management). The study results revealed no significant differences in understanding of the potential cons of alcohol use and level of alcohol use, between the precontemplation and contemplation/action clusters. Lastly, the results revealed that the precontemplation group showed significantly lower levels of trait anxiety and reported symptoms of depression. These results suggest that McConnaughy et al.'s (1983) URICA can differentiate between levels of change readiness for participants undergoing inpatient treatment for alcohol use problems. However, Willoughby and Edens' (1996) study showed that people in the precontemplation and contemplation/action clusters showed no significant difference in the alcohol treatment program completion rates.

Henderson and colleagues (2004) recruited participants for a 29-week drug abuse treatment program. Drug abuse was measured via urinalysis three times weekly throughout the program. The researchers found that stage of change scores accounted for a statistically significant proportion of the variance in heroin $\left(\Delta R^{2}=.10, p<.05\right)$ and cocaine $\left(\Delta R^{2}=.10, p<\right.$ .05) abstinence measured via urinalysis over and above participants' demographic variables, drug use severity, and treatment condition (i.e., rewards vs. no rewards for drug-free urine screens). Henderson et al. (2004) noted that treatment condition did not account for a significant change in variation of any of the outcome variables in this study over and above participants' demographic variables and drug use severity. Additionally, Henderson et al. (2004) pointed out that additional past research supports their findings. Specifically, Belding and colleagues (1997) examined urine screens for drug use for participants in a methadone maintenance program. Hierarchal regression analysis showed that, when added as a combined model, stage of change 
scores accounted for a statistically significant proportion of the variance in drug abstinence at 12week follow-up $\left(\Delta R^{2}=.14, p<.05\right)$.

\section{The TTM and Parenting Behavior}

Sherman and Carothers (2005) applied the TTM (Prochaska \& DiClemente, 1983) to the context of family therapy, suggesting that parents may present to therapy at different stages of change, which influences their readiness to participate. For instance, Sherman and Carothers (2005) characterized a precontemplative parent as someone who presents with resistance and anger, and a contemplative parent as someone who reports interest in participation but lacks follow-through. Parents in the action stage, however, show engagement (Sherman \& Carothers, 2005). In addition, past research suggested that parents who are ready for change may be more capable of identifying problems when they exist, and more willing to acknowledge that they are experiencing distress (O’Hare, 1996b; Wade \& Andrade, 2015), resulting in a stronger, positive relationship between child problems and parenting stress for parents that rate themselves as readier for change (Jones et al., 2017).

However, some researchers have questioned whether the TTM can apply consistently with issues related to parenting. For instance, Corden and Somerton (2004) have cautioned against the application of the TTM to assessment and intervention with children and families. Specifically, in an article that examined the utility and use of the TTM within the context of parental intervention and child welfare, Corden and Somerton (2004) suggested that strict application of the TTM stage model, that which they note was developed primarily to address addictive behaviors, may run the risk of being miss-appropriated to parenting behaviors. For instance, they suggested that influences on parenting behavior may be overly complex (e.g., 
context of varied cultural norms and socioeconomic demands) to fit parenting behaviors neatly within the TTM stage theory. They also questioned the usefulness of the TTM in the context of parenting and suggested that the TTM was developed to explain an individual's readiness to change in relation to their relationship with a drug, and not specifically other people in their lives. Corden and Somerton (2004) further suggested that alteration of parenting behavior, as it relates to the quality of the parent-child relationship, may not be a viable analog to the "all-ornothing" (p. 1040) quality they suggested is accompanied by the imperative of substance abstinence that may best serve individuals with drug use disorders. They suggested that changes in parenting may be better understood as incremental rather than a categorical change in parenting behavior. Lastly, they argued that the underlying assumptions about readiness to change may undermine the immediate needs of children, stating, "Children cannot wait" (Corden \& Somerton, 2004, p. 1041). The authors argued that a lack of change in cases of drug addiction, is more likely to result in personal harm, not the harm of others (e.g., their children), and suggested that readiness to change parenting behavior does not necessarily equate to actual change that would be likely to result in increased confidence in parents' ability to care for their child. However, the authors appear to levy this criticism regarding particularly damaging parent behaviors (e.g., physical and sexual abuse), and not, for example, ineffective, but not abusive discipline strategies. These points seem important when considering the potential application of the TTM in addressing the assessment needs of children and families. However, these cautionary notes are couched within the strict, stage-model application of the TTM, and may not apply necessarily to the continuous transformation of the stage-model conceptualization, a global readiness-to-change variable, that has been used in more recent research (e.g., Jones et al., 2017). 


\section{Readiness for Change and Parent Gender}

Readiness to change parenting behavior may also vary significantly in accordance with parent gender. In a study that examined child conduct behavior symptom severity, parenting stress, and parents' reported readiness to change parenting behavior in a sample of parents seeking treatment with their children, Niec and colleagues (2015) found that mothers tended to report significantly higher levels of readiness to change as compared to fathers. Additionally, the results indicated that fathers tended to significantly underreport the perceived conduct behavior symptom severity of their children in comparison to mothers. Furthermore, results indicated that mothers reported significantly higher levels of parenting self-efficacy, while fathers reported significantly more rigidity in their approach to parenting. Lastly, the results showed that there were no significant differences among parenting stress when comparing mothers and fathers, and the researchers indicated that, across the board, parents reported significantly elevated parenting stress. Niec et al.'s (2015) results showing no significant relationship between readiness to change and parenting stress run contrary to associated contemporary findings (e.g., Jones et al., 2017; Proctor et al., 2018). As a result, additional research that examines the relationship between parenting stress, readiness for change and child behavior problems is warranted.

\section{Parenting Stress and Children's Behavioral and Emotional Wellbeing}

Past research has shown a significant relationship between reported child behavior problems and perceived parenting stress (e.g., Crnic et al., 2005). For instance, Bagner et al. (2009) examined relationships between perceived parenting stress, parent-reported child externalizing behavior problems, and prenatal exposure to drugs (i.e., cocaine and/or opiates). The researchers used longitudinal data to test whether parenting stress could predict externalizing 
behavior problems. Results indicated that parents' perceived level of parenting stress, reported when their children were four months old, significantly predicted externalizing behavior problems 32 months later. More specifically, hierarchical multiple regression analysis indicated that parenting stress accounted for a significant proportion of variance in externalizing child behavior problems over and above a variety of variables (e.g., SES, child race/sex, age of the mother, and prenatal exposure to drugs). Further, the results indicated that prenatal exposure to cocaine and/or opiates did not significantly predict externalizing behavior problems over and above participant demographic variables (e.g., SES, child race/sex, and age of the mother). These findings provide support for the relationship between parenting stress and child behavior problems, specifically in regard to longitudinal data using a child sample (Bagner et al., 2009). Additionally, Liles et al. (2012) examined levels of perceived parenting stress, depression, and mother-reported child behavior problems and compared a sample of mothers with a history of prenatal methamphetamine (MA) use with a sample of non-prenatal-MA use control group. Data were collected form the mothers when their children were 36-months old. The authors found that, among these mothers, higher levels of reported child behavioral problems and higher levels of self-reported depression predicted higher levels of parenting stress (Liles et al., 2012).

Conversely, internalizing problems, specifically, child depression, has also been linked to significantly higher rates of parenting distress among mothers (Tan \& Rey, 2005). Moreover, using a sample of mothers from primarily Malay, Chinese, and Indian ethnic backgrounds, mothers with depressed children were significantly more likely than mothers without depressed children to endorse statements on the Difficult Child (DC) subscale of the Parenting Stress 
Index-Short Form (PSI-SF; see Abidin, 1990). Taken together, these findings (i.e., Jones et al., 2017; Liles et al., 2012; Tan \& Rey, 2005) suggest that parent-reported internalizing symptoms, externalizing symptoms, and behavioral problems in children are associated with higher levels of parenting stress.

Costa and colleagues (2006) examined the relationship between parent-reported parenting stress and child behavior problems (e.g., internalizing/externalizing problems) within a sample of 474 youth aged 5-17 years along with their biological parents. The authors found significant positive relationships among three of the Parenting Stress Index (PSI; see Abidin, 1995) subscales (i.e., parental distress, difficult child, and parent-child dysfunctional interactions) and both internalizing problems and externalizing problems. However, after controlling for parent reported mental health issues, some of the relationships became non-significant. The positive association between parent-child dysfunctional interactions and internalizing problems remained significant. Further, the difficult child subscale was significantly positively associated with both internalizing and externalizing problems (Costa et al., 2006). Anthony et al. (2005) added to these findings and showed that parent-reported parenting stress was related to teacher-reported internalizing and externalizing problems.

Rodriguez (2011) examined the relationships among parenting stress, children's attributional style, and internalizing problems, and found that an adaptive attributional style mediated the relationship between parenting stress and internalizing problems. Furthermore, an adaptive attributional style was significantly negatively associated with parent-reported parenting stress. Rodriguez (2011) suggested that internalizing problems for children with stressed parents may develop from negative attributions for positive events. Overall, the evidence suggested that 
parenting stress is associated with several issues including internalizing and externalizing problems (e.g., attention-deficit problems; Barry et al., 2005; Crum, \& Moreland, 2017; Stone et al., 2015; Williford et al., 2007), and lower levels of social competence (Crum, \& Moreland, 2017). Longitudinal research has also suggested that reported parenting stress decreases as children age and externalizing behavior problems may maintain parenting stress over time (Williford et al., 2007).

Overall, the research on parenting stress and externalizing child behavior problems has suggested that both of these variables likely share a bidirectional, and developmental relationship with one another, such that, in combination, parenting stress influences externalizing behavior, and externalizing behavior influences parenting stress, which authors have noted appears consistent with the transactional model of development (Barry et al., 2005; Deater-Deckard, 1998; Sameroff, 1975; Rodriguez, 2011; Stone et al., 2016). Based on these findings, Barry et al. (2005) have suggested that interventions for children and corresponding caregivers dealing with child behavior problems may also benefit from the addition of stress reduction skills training for parents in combination with child intervention techniques.

\section{Parenting Stress and Treatment Completion}

In at least one case, parenting stress has been associated with increased levels of treatment completion. Specifically, Timmer and colleagues (2004) compared kin and non-kin foster parents in regard to, among other variables, their level of parenting stress, treatment persistence, and perceived child behavior problems. Participants in this study sought to address externalizing symptoms of their foster children by participating in parent-child interaction therapy (PCIT). Results indicated that compared to non-kin foster parents, kin foster parents 
showed no significant difference in the perceived severity of their foster child's overall behavior problems (i.e., internalizing and externalizing symptoms). Furthermore, kin foster parents, compared to non-kin foster parents, were significantly more likely to finish the treatment program (Timmer et al., 2004).

\section{Parental Readiness for Change, Treatment Outcomes, and Retention}

Past research suggested that increasing parental motivation and readiness for change is associated with greater client participation and improved therapeutic outcomes. For instance, increased parental readiness for change has been associated with greater parent-child therapy attendance (Wade \& Andrade, 2015). Additionally, Chacko and colleagues (2012) found that a program focused on increasing treatment participation was associated with reduced attrition rates and increased engagement in treatment interventions for family-based therapy with children diagnosed with attention-deficit/hyperactivity disorder (ADHD). Similarly, Nock and Kazdin (2005) were able to increase treatment participation using a preparatory intervention aimed at increasing parental motivation for treatment for family-based interventions for child externalizing behavior problems. However, there appears to be some inconsistencies in the conclusions regarding motivational enhancement in family-based interventions. For example, Chaffin et al. (2009) identified some of the possible benefits of increasing motivation and decreasing attrition rates in the delivery of PCIT (e.g., greater efficiency/reduced economic burden). However, the authors noted that this effect was only found for parents who identified their pre-intervention motivation for treatment as moderate to low. Contrary to their expectations, parents who received the modified intervention and identified their pre-intervention motivation for treatment as high, showed elevated levels of attrition (Chaffin et al., 2009). 
A more recent study's results may complicate and add to the research findings described above. Specifically, Webb et al. (2017) found that modified motivational PCIT, compared to unmodified PCIT, showed no added benefit in terms of reducing dropout, and both versions were similarly helpful in reducing levels of parenting stress and child behavior problems (i.e., internalizing and externalizing behavior problems). However, the authors did find that higher pre-treatment parental readiness for change (i.e., readiness scores at 3 or above), showed lower levels of attrition compared to lower levels of pre-treatment readiness for change (i.e., readiness scores 3 or below). Additionally, Webb et al. (2017) found that participants whose readiness for change significantly improved over the course of treatment also showed reduced risk of treatment dropout. The authors concluded that parents' level of readiness for change, in the context of PCIT, may be associated with increased parent participation (Webb et al., 2017).

\section{Parenting Stress and Child Mental Health, and Readiness for Change}

Jones and colleagues (2017) examined the relationships among reported externalizing child behavior problems (e.g., attention-deficit, hyperactivity, and oppositional, and conductrelated symptoms), perceived level of parenting stress, and parents' reported readiness to change (regarding their intervention role as a parent with their children). In this study, child participants were approximately 10 years old, and the majority were boys (68\%). An assessment clinic for attention-deficit/hyperactivity disorder (ADHD) served as the data collection site, and according to the demographics from the participation sample, slightly over half of the children in the sample were reported to have had a preexisting ADHD diagnosis. Jones and colleagues (2017) revealed that parenting stress was significantly positively related to readiness for change, and that readiness for change was significantly positively associated with reported 
impulsive/hyperactive and oppositional child behavior problems. Further, results showed that parenting stress was significantly positively related to all forms of externalizing behaviors. Primary analyses showed that parenting stress scores significantly moderated the relationships among reported impulsive/hyperactive and oppositional child behavior problems and parents' readiness to change. The authors noted that impulsive/hyperactive and oppositional child behavior problems had a significantly stronger relationship to readiness for change at low levels of parenting stress, compared to high levels of parenting stress. The authors explained that the parents' reported severity of their child's behavior problems did not have a significant relationship to readiness for change for parents who reported high levels of stress. In other words, parents appear ready for change at high and low levels of child behavior problem severity. However, for parents that reported low parenting stress, readiness for change corresponded more consistently with child behavior problem severity (with greater reported severity associated with greater readiness for change). Lastly, Jones and colleagues' (2017) use of hierarchical multiple regression revealed that the interaction between impulsive/hyperactive behavior problems and parenting stress, and the interaction between oppositional child behavior problems and parenting stress both accounted for a significant proportion of the variance in readiness to change $\left(R^{2}=.19, p=.005 ; R^{2}=.17, p=.013\right.$, respectively).

Research suggested that both child behavior problems and parenting stress are significantly positively related to parents' reported readiness for change (Wade \& Andrade, 2015). Wade and Andrade (2015) conducted a validation study for a questionnaire that measures parental readiness for change, entitled the Parent Readiness for Change Scale (PRFCS). The researchers recruited parents of children/adolescents (aged 6-17 years) who were receiving 
treatment for problems related to emotional, interpersonal, and behavioral dysfunction. Confirmatory factor analysis supported a three-factor structure of the PRFCS (i.e., precontemplation, contemplation, and action). The findings further showed a significant negative correlation between the pre-contemplative and contemplative factors $(r=-.77, p<.001)$, a significant negative correlation between the pre-contemplative and action factors $(r=-.59, p<$ $.001)$, and significant positive correlation between the contemplative and action factors $(r=.79$, $p<.001)$. Secondary analysis suggested that there is a more fundamental "Readiness" factor (ranging from low readiness to high readiness). The authors stated that this factor could be measured using a transformed total score, by subtracting the pre-contemplative scale score from the combined total of contemplation and action scale scores (Wade \& Andrade, 2015).

Related, Andrade and colleagues (2015) provided an examination of the relationship between parental readiness for change, child behavior problems, and received parenting skills/behaviors. In this study, 143 parents of children identified with "disruptive" behaviors completed measures for readiness for change, their children's emotional/behavioral problems and prosocial abilities, parents' perceived level of their child's social impairment, oppositional/defiant behavior problems, and attention-deficit, impulsive, and overactive problems. The researchers found that precontemplation in regard to parenting behavior was significantly negatively associated with perceived social impairment, overall behavior problems, and positively associated with inconsistent parental discipline. On the other hand, both contemplation and action in regard to parenting behaviors, were significantly positively associated with overall behavior problems and social impairment. These results suggest that 
parents who perceive their children as having more problems are also more likely to report that they are ready for change (Andrade et al., 2015).

Another primary example of a study that identified a relationship between parental readiness for change and childhood behavior problems was conducted by Proctor and colleagues (2017). The authors set out to validate the short-form of a parental readiness measure (i.e., Readiness, Efficacy, Attributions, Defensiveness, and Importance Scale - Short Form; READISF). The authors also argued that the study may help bolster the future understanding of how readiness for change interacts with treatment retention and adherence. Proctor et al. (2017) gathered data on 128 parent-child dyads for children (aged 3 to 7 years) to examine relationships among parental motivation, readiness for change, and child behavior problems. The results of this study provided support for the validity of the READI-SF, by showing that readiness scores were associated with caregiver participation. Further, the study found that child behavior problems were significantly positively associated with overall readiness scores on the READISF. Moreover, child behavior problems were shown to explain $24 \%$ of the variance in reported overall parental readiness. These findings are consistent with the broader findings on this topic and reinforce the interpretation that higher reported child behavior problem severity is associated with greater reported parental readiness for change (Proctor et al., 2017).

Precontemplation appears to be associated with less reported child behavior problems. Littell and Girvin (2005) conducted a longitudinal study with 353 caregivers that had been referred to the Philadelphia Department of Human Services (DHS). Littell and Girvin (2005) noted that these cases had been labeled with "moderate risk" (p. 62) for recurrent abuse and/or neglect in a majority single, African American, and unemployed mother sample. Participants 
were randomly assigned to receive one of two intervention types, which included Services to Children in their Own Homes (SCOH) or family preservation services (FPS). Data were collected at three separate timepoints (i.e., 4 weeks post intervention assignment, at 16-weeks, and at 1-year). The authors measured mothers' self-reported level of depressive symptoms, an index of readiness for change regarding "problems you have in taking care of your children" (Littell \& Girvin, 2005, p. 63) using the sum of the contemplation, action, and reverse-scored precontemplation scale scores on the URICA, child behavior problems, parenting quality, social support, presence of recurrent child abuse/neglect reports, housing/economic problems, and negative/positive life events. Furthermore, at four weeks, the Problem Recognition scale (i.e., a composite of the reverse-scored precontemplation scale on the URICA) was significantly positively related to child behavior problems, and the Intentions to Change scale (i.e., a composite of the contemplation and action scales on the URICA) was negatively related to child behavior problems. These findings suggest that parents who are pre-contemplative of problems regarding parenting tend to report fewer child behavior problems compared to parents better characterized by contemplation and action regarding these problems (Littell \& Girvin, 2005).

Relevant to the current study, the results of Wade and Andrade's (2015) research indicated that parental readiness to change was significantly related to emotional and behavioral problems among children/adolescents. Specifically, both internalizing and externalizing problems showed a significant negative association with the precontemplation factor, and significant positive associations with the contemplation and action factors. Results also showed that lower levels of reported inconsistency in delivering parental discipline was associated with lower levels of readiness for change. The authors suggested a couple explanations: either, a 
parent's readiness for change is reflected by their child's level of behavior problems (with readiness increasing as behavior problems worsen), or readiness quality has an impact on how parents interpret their child's behavior (either as less severe among low readiness parents, or more severe among high readiness parents). The readiness to change model has also been applied within the context of the family. For instance, Miller and colleagues (2016) noted that parents characterized by pre-contemplation may be likely to say something along the lines of, "I don't have the problem, it's all my kid's fault" (p. 89) when faced with their role in addressing and understanding their child's behavior problems.

\section{Focus of The Current Study}

To expand on this burgeoning new area of research, I attempted to replicate Jones and colleagues' (2017) initial findings (i.e., parenting stress as a moderator of the relationship between externalizing behavior problems and parental readiness for change) and contribute a novel expansion. Specifically, I examined parenting stress as a potential moderator of the relationship between internalizing behavior problems and parental readiness for change.

In the current study, I have drawn on the Transtheoretical Model of Behavior Change (TTM; DiClemente, \& Prochaska, 1982; Prochaska, \& DiClemente, 1982, 1983; Prochaska et al., 1985), which has been used as an explanatory model to understand the relationship between child behavior problems and parental readiness for change in regard to their parenting behaviors for children and families seeking mental health treatment (Jones et al., 2017). Moreover, according to the TTM, individuals vary in their readiness for change. For instance, individuals who are pre-contemplative of a problem, have not considered that their behavior is problematic and have not considered changing their behavior. In contrast, individuals who show greater 
levels of readiness to change (i.e., report qualities consistent with contemplative, action, or maintenance stages), do exhibit readiness for change, and have undergone the process of recognizing, considering, acting towards, or trying to preserve behavioral changes (DiClemente, \& Prochaska, 1982; Prochaska et al., 1985; Prochaska, \& DiClemente, 1982, 1983).

For the current study, I applied a moderation model to explain the relationships between child behavior problems, parenting stress, and parental readiness for change in the same way described in Jones et al. (2017). A mediation model was also considered for the current study. However, the moderation model was ultimately decided upon. This decision was made for a few reasons. First, while both child behavior problems and parenting stress have been associated with parental readiness for change (e.g., Jones et al., 2017; Proctor et al., 2017; Wade \& Andrade 2015), a predicted causal process is not well explained by the TTM (DiClemente, \& Prochaska, 1982; Prochaska, \& DiClemente, 1982, 1983; Prochaska et al., 1985). Moreover, as suggested by Miller and colleagues (2016), a parent may present as both stressed, and indicate that their child has problems, but report that they have no intention to change because they state that the problem lies within their child.

Additionally, the TTM (DiClemente, \& Prochaska, 1982; Prochaska, \& DiClemente, 1982, 1983; Prochaska et al., 1985) conceptualizes readiness for change as an individual, cognitive process that may not be fully explained by the behavior of individuals outside of the self (Corden \& Somerton, 2004). As a result, I conceptualize parenting stress as a factor affecting the relationship between child behavior problems (both internalizing and externalizing) and readiness for change, which is consistent with previous research (i.e., Jones et al., 2017). The current study was conducted as an additional examination of this model and to also explain this 
relationship within the context of internalizing problems. Lastly, the mediator model appeared less applicable to parenting stress and child behavior problems because the causal order of these two variables may be unclear. For instance, past evidence suggested that the relationship between these two variables is bidirectional, and that they develop over time in relation to each other, and not as a unidirectional process (e.g., parenting stress uniquely leads to behavior problems or vice versa; Stone et al., 2016).

Recent findings also suggested that parenting stress may serve as a potential moderator of the relationship between externalizing child behavior problems and readiness for change (Jones et al., 2017). Jones et al. (2017) also argued that parents who are stressed may also perceive themselves both as less competent (Chi et al., 2015; Cunningham \& Boyle, 2002; Renner et al., 2015), and less self-efficacious parents (Bloomfield \& Kendall, 2012; Heath et al., 2015; May et al., 2015). For the reasons discussed above, the moderation model was used to explain the strength of the relationships among parenting stress, child behavioral/emotional problems, and parental readiness for change (see Figure 1).

\section{Figure 1}

Predicted moderation model of the relationship between internalizing and externalizing child emotional and behavioral problems and readiness to change parenting behavior

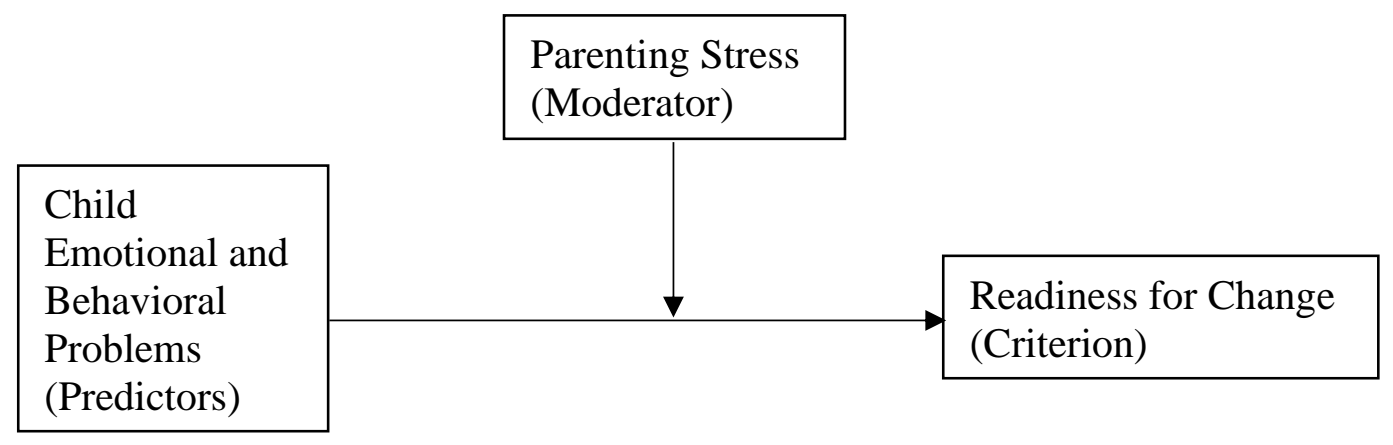




\section{Research Question}

1. Does parenting stress moderate the relationship between internalizing and externalizing child mental health problems, and parental readiness for change?

\section{Hypotheses}

1. There will be a significant positive correlation between internalizing child mental health problems and parental readiness for change.

2. There will be a significant positive correlation between externalizing child mental health problems and parental readiness for change.

3. There will be a significant positive correlation between parenting stress and parental readiness for change.

4. The relationship between internalizing problems and parental readiness for change will be moderated by parenting stress, such that at low levels of parenting stress, there will be a significant positive relationship between internalizing problems and parental readiness for change. Conversely, at high levels of parenting stress, there will be no significant relationship between internalizing problems and parental readiness for change (i.e., readiness will remain elevated at both low and high levels of internalizing problems; see Figure 2 below). 


\section{Figure 2}

Predicted moderation model of the relationship between internalizing child mental health problems and readiness to change parenting behavior

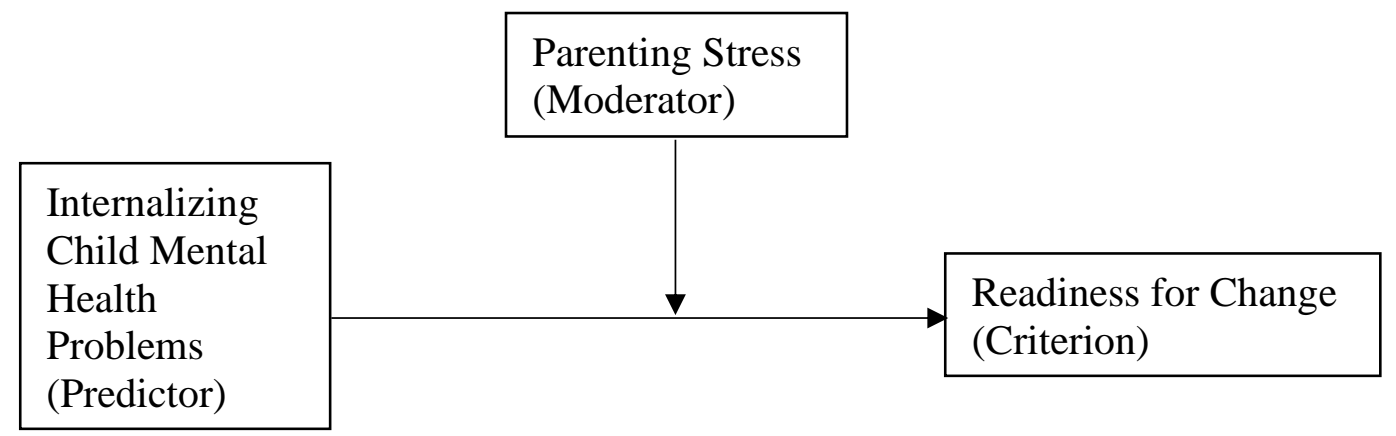

5. The relationship between externalizing problems and parental readiness for change will be moderated by parenting stress, such that at low levels of parenting stress, there will be a significant positive relationship between externalizing problems and parental readiness for change. Conversely, at high levels of parenting stress, and there will be no significant relationship between externalizing problems and parental readiness for change (i.e., readiness will remain elevated at both low and high levels of externalizing problems; see Figure 3 below). 


\section{Figure 3}

Predicted moderation model of the relationship between externalizing child mental health problems and readiness to change parenting behavior

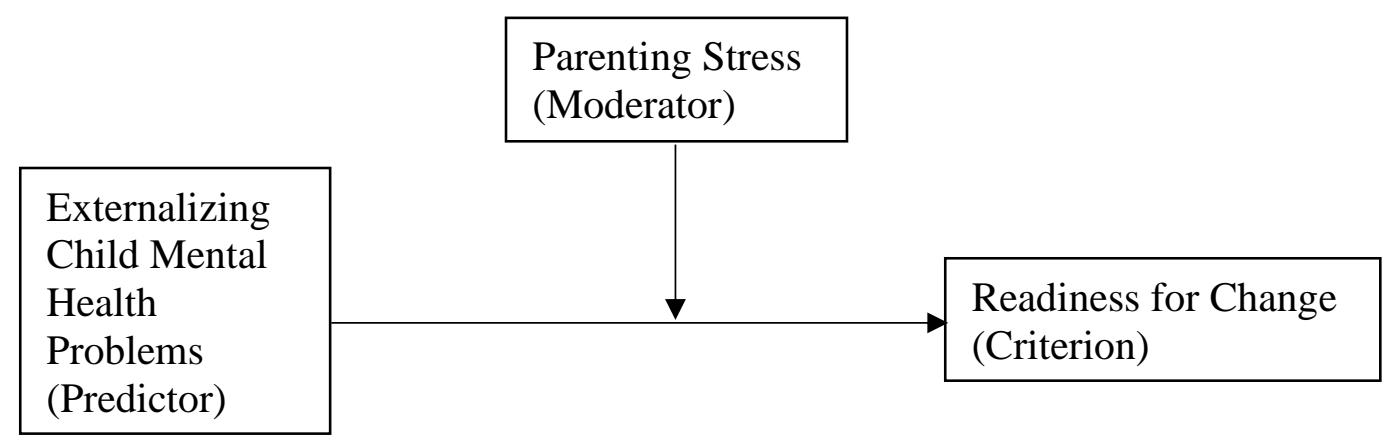




\section{CHAPTER 3: METHOD}

\section{Research Design}

The current study used a quantitative, cross-sectional, survey-style research design to investigate parenting stress as a moderator of the relationship between child internalizing and externalizing problems and parental readiness for change. All the data in the current study was self-reported by caregivers. Furthermore, internalizing and externalizing child mental health problems were used as separate predictor variables using two multiple linear regression tests of moderation. For each, parenting stress was included as the moderator variable of the relationship between both predictor variables and the sole outcome variable, parental readiness for change (Aiken, \& West, 1991). Participants completed the demographics forms in addition to study measures, the Behavior Assessment System for Children, Third Edition (BASC-3), Parenting Stress Index, Fourth Edition (PSI-4), Parenting Stress Index, Fourth Edition Short-Form (PSI-4SF), and the Parenting Readiness for Change Scale (PRFCS).

\section{Participants}

Participants for the current study included parents and legal guardians who completed the intake paperwork to enroll their child in mental health services. Measures were completed by one parent or legal guardian during the intake interview and were based on which adult had accompanied the client. If a guardian completed assessments for multiple children upon intake, only the first child's data entered into the dataset was used for this study's data analysis. The participant's relationship to the client was collected as part of the demographics data to be included in the preliminary analyses. The data was collected at a medium-sized outpatient child, adolescent and family therapy clinic in the Appalachian region. This convenience sample was 
comprised of participants 18 years of age or older. Demographics information was collected during the intake session.

An a priori power analysis was computed using G*Power software (Faul et al., 2009) to estimate the necessary sample size needed to examine a significant effect if one exists. For the G*Power analysis, alpha $(\alpha)$ was set at .05 , with a power $(1-\beta=.80)$ and an estimated medium effect size (.15; Cohen, 1988). The results of this power analysis indicated that a minimum of 77 participants would be needed to find a significant effect. This sample estimate was considered feasible pre-pandemic because the medium-sized outpatient clinic had typically served approximately 200 or more clients per year. The total number of participants for the current study totaled 64.

\section{Measures}

Parent Readiness for Change Scale. The Parent Readiness for Change Scale (PRFCS) was developed to measure parental readiness to change their parenting behavior (Brestan et al., 1999). For the current study, a shortened, 17-item version of the PRFCS was used (i.e., the Parent Readiness for Change Scale - Short Form; PRFCS-SF), which has recent support as a valid measure of parental readiness for change (Wade \& Andrade, 2015). The PRFCS-SF assesses parental readiness for change using a 5-point, Likert scale which ranges from 1 (Strongly Disagree) to 5 (Strongly Agree). An example item for this measure includes: “As far as I'm concerned, I don't need to change how I take care of my child” (precontemplation). Consistent with suggestions made by Wade and Andrade (2015), and others (e.g., DiClemente et al., 1999; Shields \& Hufford, 2005), the current study computed an overall parental readiness for change score by summing subscores for contemplation and action scales and then deducting 
precontemplation subscores. The resulting score served as an overall parental readiness score. Potential scores for this measure range from -13 to 55, with higher scores indicating higher levels of parental readiness for change (Brestan, et al., 1999). Based on the study results, each participant's score was transformed by adding 12 points to each participants' composite score to avoid potential negative values for overall parental readiness for change. Similar methods of producing an overall readiness index have been used in previous research (e.g., Boswell et al., 2012; Littell \& Girvin, 2005), and have been suggested as an alternative to separate scores for each stage of change (Wade \& Andrade, 2015).

Wade and Andrade (2015) noted that the PFRCS is based theoretically on the TTM and was originally constructed using items from the URICA questionnaire (Brestan, et al., 1999; McConnaughy et al., 1989; Prochaska \& DiClemente, 1992). The authors have indicated that the PRFCS may serve as a helpful screening measure for parents of children receiving clinical services due to its ability to assess for parental readiness for change. Research has shown good support for the internal consistency reliability of the PRFCS. Specifically, alpha $(\alpha)$ values for the precontemplation subscale ranged .70-.78, for contemplation ranged .82-.87, and action ranged .73-.80 (Andrade et al., 2015; Niec et al., 2015; Wade \& Andrade, 2015). Additionally, there is support for the PRFCS as a valid measure of parental readiness for change. For instance, Wade and Andrade (2015) found that parental readiness, as measured by the PRFCS, was significantly positively related to parent report of child emotional and behavioral problems. In other words, parents who perceived more problems tended to report greater readiness for change. Additionally, parents' perceived inconsistencies in their discipline were significantly positively associated with contemplation scores, and significantly negatively associated with 
precontemplation scores. This suggests that the PRFCS can tap clinically relevant indicators of parental readiness. Lastly, higher global readiness among parents significantly predicted greater treatment participation, indicating that parental readiness for change, as measured by the PRFCS was related to increased treatment adherence (Wade \& Andrade, 2015).

Confirmatory factor analysis indicated that the 17-items significantly loaded onto their proposed factor (i.e., precontemplation, contemplation, and action) and scores on the PRFCS-SF accounted for approximately $37 \%$ of the variance in parent reported emotional/behavioral problems, parenting skills, and treatment attendance (Wade \& Andrade, 2015). Wade and Andrade's (2015) results also indicated that both the contemplation and action factors were significantly positively related to one another, and that both were significantly negatively related to the precontemplation factor (all $p s<.001$ ). The results of this study also found greater support for a "superordinate" readiness factor in comparison to the three-factor structure. This indicates that parenting readiness for change is better conceptualized as a single, global readiness factor. Moreover, the authors found that they could avoid impractical and complex computation and significantly approximate this global readiness factor $(r=.96, p<.001)$ by combining the scores on contemplation and action scales, and then deducting the precontemplation scores (Wade \& Andrade, 2015). The internal consistency reliability (Cronbach's alpha values) estimates for this measure could not be computed for the measures in the current sample because the clinical database for the study only contained total scores and did not include individual item responses; see limitations section for more details.

Parenting Stress. Parenting stress will be measured using the Parenting Stress IndexShort Form, 4th edition (PSI-4-SF), Parenting Stress Index-Long Form, 4th edition (Abidin, 
1995; Abidin, 2012), and the Stress Index for Parents of Adolescents (SIPA; Sheras et al.,1998). The Parenting Stress Index-Long Form, 4th edition is a 120-item measure for guardians with children aged one month to 12 years of age and takes approximately 20 minutes to complete. The test re-test reliability for the total stress score ranges from .65 to .96. Internal consistency reliability coefficients ( $\alpha$ 's) of its two primary domains are greater than or equal to .96 . and has shown adequate validity across various clinical populations (Abidin, 1995; Abidin, 2012; PAR Inc., n.d.).

The Parenting Stress Index-Short Form, 4th edition (PSI-4-SF; Abidin, 2012) consists of 36 items (out of the 120-item, full length version) and has three primary domains (i.e., Parental Distress, PD; Parent-Child Dysfunctional Interaction, P-CDI; and Difficult Child, DC), and a secondary, global domain, Total Stress scale. The PSI-4-SF utilizes a norm-referenced, nationally-stratified sample based on the 2007 United States Census for scoring procedures, and results provide the raw scores, percentile rank, and $T$-scores for each subscale within the Child and Parent domains, along with the Total Stress Score, with $T$-scores above the 90th percentile indicated as "clinically significant." This measure was designed for use with children (aged one month to 12 years) and is a self-report measure completed by parents, taking approximately 10 minutes to complete. Participants endorse items using a 5-point Likert scale that ranges from 1 (strongly agree) to 5 (strongly disagree). In a few cases, participants are asked to indicate their agreement to graded statements instead of the 5-point Likert-style item response format. For instance, participants are asked to select a response to the following item: "I feel that I am: 1 . Not a very good parent; 2 . A person who has some trouble being a parent; 3 . An average parent; 4. A better than average parent; 5. A very good parent." 
The PSI-4-SF also has strong support for both reliability and validity. Internal consistency reliability coefficients ( $\alpha^{\prime}$ s) of its three primary domains have been found to range from .88 to .90 , with a test-retest reliability of .84 . Furthermore, scores on the PSI-4-SF are highly correlated with the third version of the Parenting Stress Index, Short-Form $(r=.99$; Abidin, 2012). Thomson and colleagues (2018) reported strong internal consistency for this measure $(\alpha=.94)$. Past research has also suggested that the PSI-4-SF has good construct validity. For instance, Phillips and colleagues (2017) found that mothers of children with Down syndrome reported significantly higher total stress scores than mothers of children with typical development (TD). Additionally, parents of children with fetal alcohol spectrum disorders (FASD) who reported higher levels of parenting distress also reported lower levels of parenting satisfaction (Petrenko et al., 2016). Furthermore, parents who participated in an 8-week mindfulness-based parent-training program showed significant reductions in their parent-child dysfunctional interaction, parental distress, and total stress scores (Anderson \& Guthery, 2015). Lastly, Thomson and colleagues (2018) found the PSI-SF-4 to be positively associated $(r=.57)$ with the Stress subscale of the Depression, Anxiety and Stress Scale (DASS21; Lovibond, \& Lovibond, 1995). The PSI-SF-4 is a proprietary measure. Therefore, it cannot be reproduced in full in this manuscript, and has been excluded from the appendixes in this document.

Stress Index for Parents of Adolescents (SIPA; Sheras et al., 1998) measures parenting stress for parents of adolescents aged 11-19. The SIPA is a 112-item measure that takes approximately 20 minutes to complete and has 5-point Likert-style response scales for each item. Items load onto three domains: Adolescent, Parent, and the Adolescent Parent Relationship. For the purposes of the current study, the Total Stress (TS) score was used. Sheras et al. (1998) 
reported strong internal consistency reliability among its three domains and total stress scores (i.e., $\alpha s>$.90). Recent studies have also shown support for the validity of the SIPA. For example, parents with a child diagnosed with Attention-Deficit/Hyperactivity Disorder (ADHD) or Autism Spectrum Disorder reported greater parenting stress compared to control groups (Hutchison et al., 2016; Wiener et al., 2016). Research has also indicated that parenting stress was significantly correlated with child behavior problems (Melançon et al., 2019).

The PSI-4 Short form, PSI-4 Long form, and SIPA measures assess parenting stress at different periods of child development. The SIPA has been considered a "developmentally appropriate extension of the PSI" (Hutchison et al., p. 3648, 2016; Sheras et al., 1998). Per Hutchison and colleagues (2016), a total stress score for the SIPA and PSI-4 forms was computed into a combined standardized parenting stress score (PS-TOT). In the current study, the SIPA and PSI-4 percentile rank scores were dichotomized into "high" (i.e., percentile rank 85 and above) and "low" (percentile rank 84 and below) parenting stress in order to use parenting stress as a moderator variable in the moderation analysis. In the current study, 59 participants fell in the "low" stress category (92.2\% of the sample) and 5 participants fell in the "high" stress category ( $7.8 \%$ of the sample). The percentile rank of 85 and above/below was used to demark between typical parenting stress levels and "borderline" or "high" levels of parenting stress that are clinically relevant. This score is consistent for "borderline" or "high" levels of parenting stress on the PSI-4 and SIPA.

The internal consistency reliability (Cronbach's alpha values) estimates for this measure could not be computed for the measures in the current sample because the clinical database for 
the study only contained total scores and did not include individual item responses; see limitations section for more details.

\section{Behavior Assessment System for Children, Third Edition (BASC-3; Reynolds \&}

Kamphaus, 2015). The Behavior Assessment System for Children, Third Edition (BASC-3; Reynolds \& Kamphaus, 2015) is a child and adolescent self- and other-report assessment measure for behavioral and emotional problems. For the current study, the BASC-3 was used as a caregiver report measure of their child's internalizing and externalizing problems. The BASC-3 has a total of nine primary clinical scales (i.e., hyperactivity, aggression, conduct problems, anxiety, depression, somatization, attention problems, atypicality, and withdrawal), with three corresponding composite scales (i.e., externalizing problems and internalizing problems). The for the current study, the BASC-3's parent-report measure (PRS) was used. The BASC-3 is appropriate for children and adolescents aged 2 to 21 years and takes approximately 10-20 minutes to complete (BASC-3 Publication Summary, n.d.; Reynolds \& Kamphaus, 2015). For the current study, $T$-scores for the externalizing problems (EP) and internalizing problems (IP) subscales were used (BASC-3 Publication Summary, n.d.; Reynolds \& Kamphaus, 2015).

The BASC-3 was developed using nationally-representative data from 44 U.S. states with 1,800 youth aged 2-21 years. These data were gathered between April 2013 and November 2014 and allow results to be transformed into normed $T$-scores. A $T$-score below 60 represents the normal range of functioning for the clinical scales and composite scores. A $T$-score ranging from 60-69 represents an "at risk" level of functioning, and a $T$-score at or above 70 represents a “clinically significant” level of risk (BASC-3 Publication Summary, n.d.; Reynolds \& Kamphaus, 2015). Percentile ranks, and 90\% Confidence Intervals are also computed by the Q- 
global $^{\mathrm{TM}}$ online scoring system (Pearson Education, 2016) and are available as a part of the interpretive summary report.

The PRS shows good internal consistency reliability across clinical and adaptive scales. Alpha coefficients for the PRS composite scores ranged from .93 to .97, and the alpha coefficients for clinical and adaptive scales ranged from .83 to .89 across age groups. Median test-retest reliability estimates for PRS composite scores ranged from .88 to .93 , and the clinical and adaptive scales ranged from .85 to .90 across age groups and median interrater reliability estimates for the PRS composite scores ranged from .73 to .82 across age groups. Specific to the current study, the internalizing and externalizing problems composites have shown to have good internal consistency reliability $(\alpha=.92$ and $\alpha=.95$, respectively; BASC-3 Publication Summary, n.d.; Reynolds \& Kamphaus, 2015). The BASC-3 is established as a valid assessment of child and adolescent emotional and behavioral functioning based on past correlation studies conducted as part of its initial development (BASC-3 Publication Summary, n.d.; Reynolds \& Kamphaus, 2015). The BASC-3 is a proprietary measure. Therefore, it cannot be reproduced in full in this manuscript, and has been excluded from the appendixes.

The internal consistency reliability (Cronbach's alpha values) estimates for this measure could not be computed for the measures in the current sample because the clinical database for the study only contained total scores and did not include individual item responses; see limitations section for more details.

Demographic Questionnaires. The Demographic forms (Appendix D) used in the study were from a medium, outpatient individual and family therapy clinic. These documents included the General Child/Adolescent Information Form, the General Adult Information Form, and 
Child/Adolescent Therapy Questionnaire - Supplement Form. Among other questions, these demographic forms gathered data on parent and child ages, child's primary language, parent age(s), the reporter's relationship to the child, child race (i.e., African-American, Asian, BiRacial, Hispanic/Latino, White or Other), household income (i.e., Less than $\$ 10,000, \$ 10,000-$ $\$ 19,000, \$ 20,000-\$ 29,000, \$ 30,000-\$ 39,000, \$ 40,000-\$ 49,000, \$ 50,000-\$ 59,000$, and 60,000 or Greater) an indication of open family court case (i.e., Yes or No), current child protective services involvement (i.e., Yes or No), parental marital status (e.g., Divorced) and parent's educational level (i.e., Grade School, Middle School, High School, Trade School, Undergraduate College Education, Master's Program in College, Doctoral Program in College, or Other). These demographic forms were given to clients as part of routine intake paperwork and information gathering. For the current study, the following demographic variables were used: Child Age, Child Sex, Child Race, Guardian Age, Guardian Sex, Guardian Relationship to Child, Guardian Race, Guardian Years in School, Guardian Marital Status, Household Income, Type of Allegation and Guardian Relationship to Child.

\section{Procedure}

Following approval from the Institutional Review Board (IRB) on 02/25/2021 (see Appendix A), participant data for the current study were collected as archival data. The clinical data included self-report by parents and legal guardians on demographic variables, parenting stress (PSI-4 and SIPA), parental readiness for change (PRFCS-SF), and perceived child behavioral and emotional problems (BASC-3 externalizing and internalizing composite scores). 


\section{Recruitment}

The current study used archival data from a convenience clinical sample of clients at a medium outpatient child, adolescent, and family therapy clinic. These data were collected as part of a separate clinical program initiative focused on informing therapeutic interventions for children and parents that have been impacted by parental opiate addiction. Parents and guardians 18 years of age or more who completed the necessary measures (i.e., BASC-3, PSI-4, SIPA, and PRFCS-SF) as part of their clinical intake and who agreed to participate in the study were included. Data were collected over a 24-month period from June of 2019 through July of 2021.

\section{Data Collection}

The current study included archival data from clinical measures (BASC-3, PSI-4

Long/Short forms, PRFCS-SF, SIPA and demographics forms) administered by either clinic staff or undergraduate student volunteers. All instruments were paper-and-pencil format. These measures were provided to clients on a routine basis as part of parenting and new client orientations. Participant data was uploaded to SPSS version 27 software (SPSS) to perform the statistical analyses necessary to evaluate the current study's hypotheses.

Raw scores for the BASC-3 and PSI-4 were entered manually by staff or undergraduate student volunteers into online scoring systems (i.e., via PARiConnect ${ }^{\mathrm{TM}}$ and Q-global ${ }^{\mathrm{TM}}$, respectively). The PRFCS-SF was hand-scored. Raw scores from this variable were transformed by subtracting participants' pre-contemplative scale scores from the sum of their contemplation and action scores on the PRFCS-SF using an excel formula document by a clinic member before the data was entered into the encrypted database. Similar methods of computing an overall readiness composite have been used in previous research (i.e., Boswell et al., 2012; Littell \& 
Girvin, 2005) and was specifically endorsed as a method for examining overall parental readiness with the PRFCS-SF measure in a recent construct validation study (i.e., Wade \& Andrade, 2015). To avoid negative values for overall parental readiness for change, each score was transformed by adding 12 points to each participants' composite score. This resulted in readiness for change scores that ranged from 0 to 54, with higher scores indicating more readiness to change parenting behavior. In total, eight participants completed the PSI-Short form, 38 participants completed the PSI Long form, and 18 participants completed the SIPA. These data were collected between June 2019 and July 2021.

Results for the SIPA were computed by hand initially but were later transferred to an online scoring system. For the current study, participant responses were deidentified and compiled into an encrypted HIPAA compliant electronic database. Each participant was be assigned a deidentified participant number, and their data manually into a SPSS datasheet stored on an encrypted database.

\section{Preliminary Analyses}

For the preliminary analyses, descriptive statistics for the sample demographics were computed. Pearson or Spearman bivariate correlations were computed between the continuous demographic variables and the criterion variable (parental readiness for change) and a $t$-test, oneway analysis of variance (ANOVA), or Kruskal-Wallis H was computed for categorical demographic variables to identify any significant differences among demographic variables in relation to the criterion variable (parental readiness for change). If any significant relationships or differences were found, the variables were included as covariates in the primary analyses. 


\section{Tests of Statistical Assumptions}

Preliminary tests were conducted to determine if any statistical assumptions associated with multiple linear regression have been violated (e.g., outliers, multicollinearity, normal distribution of residuals, non-linear relationship between predictor and outcome variable, homoscedasticity, and independence of observations). To identify a potential violation of multicollinearity the variance inflation factor (VIF) was examined. A VIF greater than 10 indicated that the multicollinearity assumption has violated (Cohen et al., 2003). To assess for normality, homoscedasticity and linearity, scatter plots and the Shapiro-Wilk test were computed and examined. To test for independence of observations, the Durbin-Watson statistic (Durbin, \& Watson, 1951). A Durbin-Watson statistic between 1.5 and 2.5 would indicate that independence of observations has not been violated (Dufour, \& Dagenais, 1985; Field, 2013). Outliers, leverage values, and influential cases were assessed by identifying studentized deleted above or below three standard deviations (Cohen et al., 2003), leverage values were compared to the leverage value cutoff using the formula $3 \mathrm{p} / \mathrm{n}$, where $p=$ number of parameters plus intercept and $n=$ number of participants (Cohen et al., 2003; Kutner et al., 2005), and influential cases were identified using Cook's distance (Cook \& Weisberg, 1982). Lastly, I created mean-centered predictor variables to use for the moderated regression analyses to aid in the interpretation of findings (Dawson, 2014). 


\section{CHAPTER 4: RESULTS}

\section{Preliminary Analyses}

Preliminary analyses were conducted to determine if any study demographic variables were statistically related to the outcome variable, parental readiness for change. Study demographic data, means, standard deviations, and bivariate correlations among clinical variables were produced (see below). Assumptions testing was conducted prior to each analysis and is detailed below.

Table 1

Child and Guardian Demographics

Mean $n(\%)$

$(S D)$

Child Demographics

Age

9.0

Sex

Female

(3.66)

Male

$38(59.4)$

$26(40.6)$

Race*

African American

$3(4.7)$

Bi-Racial

$3(4.7)$

Hispanic/Latino

$1(1.6)$

White

$56(87.5)$

Guardian Demographics

Age

41.58

(10.36)

Sex

Female

$48(75.0)$

Male

$16(25.0)$

Race

African American

$1(1.6)$

Bi-Racial

$3(4.7)$

Hispanic/Latino

1 (1.6)

White

$59(92.2)$ 
Guardian's Relationship to Child

Guardian's Marital Status*
Biological Father

Biological Mother

Foster Father

Foster Mother

Grandfather

Grandmother

Uncle

Aunt

Other

Single

Married

Divorced

Separated

Other

Middle school

High school

Trade school

Undergraduate college

education

Master's program in college

Doctoral program in college

Other

Less than $\$ 10,000$

$\$ 10,000-\$ 19,000$

$\$ 20,000-\$ 29,000$

$\$ 30,000-\$ 39,000$

$\$ 40,000-\$ 49,000$

$\$ 50,000-\$ 59,000$

$\$ 60,000$ or Greater

Yes

No

Yes

No

Child Sexual Abuse/Assault Domestic and/or Family

Violence
10 (15.6)

27 (42.2)

$2(3.1)$

$4(6.3)$

$1(1.6)$

$11(17.2)$

$2(3.1)$

$3(4.7)$

$4(6.3)$

17 (26.6)

17 (26.6)

$18(28.1)$

$6(9.4)$

$5(7.8)$

$3(4.7)$

$21(32.8)$

$5(7.8)$

$11(17.2)$

$5(7.8)$

$3(4.7)$

$13(20.3)$

9 (14.1)

10 (15.6)

$11(17.2)$

$6(9.4)$

$6(9.4)$

7 (10.9)

$11(17.2)$

26 (40.6)

38 (59.4)

27 (42.2)

37 (57.8)

17 (26.6)

1 (1.6) 
Drug-Exposed

$11(17.2)$

Sexual Exploitation

$1(1.6)$

Witness to Domestic Violence

$1(1.6)$

Child Physical Abuse

$3(4.7)$

Multiple Categories

$6(9.6)$

Missing

$24(37.5)$

Note. * $N$ varies due to missing values. Reproduced in Appendix D.

\section{Table 2}

Means, standard deviations, and bivariate correlations among Parental Readiness for Change, Parenting stress, Internalizing Problems, and Externalizing Problems

\begin{tabular}{lllll}
\hline Variable & 1. & 2. & 3. & 4. \\
\hline M & 29.01 & 52.83 & 55.97 & 57.23 \\
SD & 11.55 & 24.75 & 13.03 & 12.85 \\
1. Readiness for Change & -- & & & \\
2. Parenting Stress & $.422^{* *}$ & -- & & \\
3. Internalizing Problems & $.295^{*}$ & $.508^{* *}$ & -- & \\
4. Externalizing Problems & $.356^{* *}$ & $.636^{* *}$ & $.599 * *$ & --
\end{tabular}

Note. Spearman's Rho was used for Externalizing Problems correlations and Pearson's r was used for Readiness, Parenting Stress and Internalizing Problems. $*<.05 . * *<.001$. Reproduced in Appendix E.

Child Age. For the current study, child age averaged 9 years $(S D=3.67)$ and ranged from 3 to 18 years. A Pearson Correlation was computed to determine if there was a statistically significant relationship between child age and parental readiness for change. The data was examined prior to analysis to determine whether there was a linear relationship between child 
age and parental readiness for change, identify potential outliers, and examine the data to determine if variables were approximately normally distributed. Visual examination of a scatter plot suggested that a linear relationship exists between child age and parental readiness for change. Next, visual examination of a scatter plot suggested that there were no outliers present. To test for normality, the Shapiro-Wilk test was computed. These results showed that parental readiness for change was approximately normally distributed $(p=.374)$. However, child age was not normally distributed $(p=.048)$. Instead, a Spearman's correlation was computed (Laerd Statistics, 2018; Privitera, 2015). The result showed a significant, negative relationship between child age and parental readiness for change, $r_{s}(64)=-.415, p<.001$. As a result, child age was entered as a covariate in model 1 for the multiple hierarchal linear regression analyses for hypotheses 4 and 5, and again for the main effects analysis.

Guardian Age. For the current study, guardian age averaged 41.58 years $(S D=10.36)$ and ranged from 25 to 67 years. A Pearson Correlation was computed to determine if there was a statistically significant relationship between guardian age and parental readiness for change. The data was examined prior to analysis to determine if a linear relationship exists between guardian age and parental readiness for change, potential outliers were identified, and the data was examined to determine if these variables are approximately normally distributed. Visual examination of a scatter plot suggested that a linear relationship exists between child age and parental readiness for change. Next, visual examination of a scatter plot suggested that there were no outliers present. To test for normality, the Shapiro-Wilk test was computed. These results showed that both guardian age and parental readiness for change are approximately normally distributed (both $p s>.05$ ). A Pearson's bivariate correlation was then computed. The 
result showed no significant relationship between guardian age and parental readiness for change $(r=-.122, p=.337)$. As a result, guardian age was not entered as a covariate in any subsequent multiple hierarchal linear regression analyses.

Child Sex. An independent samples $t$-test was computed to determine if there are statistically significant differences between child sex groups (i.e., male and female) regarding parental readiness for change. Examination of a boxplot indicated that there was one outlier. This data point was filtered out prior to the analysis. The Shapiro-Wilk's test indicated that parental readiness for change was approximately normally distributed $(p>.05)$. Levene's test for equality of variances indicated that there was homogeneity of variances for parental readiness for change for male $(n=26)$ and female $(n=38)$ children $(p=.652)$. There was no statistically significant difference in parental readiness for change between male $(M=31.15, S D=12.27)$ and female $(M$ $=28.29, S D=10.09)$ children, $t(61)=1.01, p=.316$. As a result, child sex was not entered as a covariate in any subsequent multiple hierarchal linear regression analyses.

Guardian Sex. An independent samples $t$-test was computed to determine if there are statistically significant differences between guardian sex (i.e., male and female) regarding parental readiness for change. Examination of a boxplot indicated that there were no outliers. The Shapiro-Wilk's test indicated that parental readiness for change was approximately normally distributed $(p>.05)$. Levene's test for equality of variances indicated that there was homogeneity of variances for parental readiness for change for male $(n=16)$ and female $(n=48)$ guardians $(p=.673)$. There was no statistically significant difference in parental readiness for change between male $(M=26.06, S D=10.60)$ and female $(M=30.00, S D=11.79)$ guardians, $t(62)=$ - 
$1.08, p=.241$. As a result, guardian sex was not entered as a covariate in any subsequent multiple hierarchal linear regression analyses.

Open Family Court Case. An independent samples $t$-test was computed to determine if there are statistically significant differences between family court case (FCC) status groups (i.e., yes or no) regarding parental readiness for change. Examination of a boxplot indicated that there were no outliers. The Shapiro-Wilk's test indicated that parental readiness for change was approximately normally distributed across open family court case status $(p s>.05)$. Levene's test for equality of variances indicated that there was homogeneity of variances for parental readiness for FCC children $(n=27)$ and children with no FCC children $(n=37), p=.113$. The result showed a significant difference in parental readiness for change between open FCC $(M=$ 32.67.15, $S D=9.31)$ and no FFC children $(M=26.35, S D=12.39)$ children, $t(62)=-2.23, p=$ .03. As a result, FCC status was entered as a covariate in subsequent multiple hierarchal linear regression analyses.

Open CPS Case. An independent samples $t$-test was computed to determine if there are statistically significant differences between open CPS case groups (i.e., yes or no) regarding parental readiness for change. Examination of a boxplot indicated that there were three outliers. These data points were filtered out prior to the analysis. The Shapiro-Wilk's test indicated that parental readiness for change was approximately normally distributed across CPS case status groups $(p s>.05)$. Levene's test for equality of variances indicated that there was not homogeneity of variances for parental readiness for change for Open CPS case $(n=24)$ and no open CPS case $(n=37)$ children $(p=.037)$. There was no statistically significant difference in parental readiness for change between open CPS case $(M=29.87, S D=7.46)$ and no Open CPS 
case $(M=29.22, S D=12.18)$ children, $t(59)=-.262, p=.794$. As a result, Open CPS Case was not entered as a covariate in any subsequent multiple hierarchal linear regression analyses.

Child Race. Child race had a total of four groups: African American $(n=3)$, Bi-Racial ( $n$ $=2)$, Hispanic/Latino $(n=1)$, and White $(n=52)$. Normality data could not be computed for parental readiness for change on all levels of child race because the "Bi-Racial" category only had two participants, and the "Hispanic/Latino" category only had one participant. A KruskalWallis $\mathrm{H}$ test was computed as a non-parametric alternative to a one-way Analysis of Variance (ANOVA) to determine if there was a significant difference in parental readiness for change scores between child race groups. The results of the Kruskal-Wallis H test suggested the distributions of parental readiness scores across levels of child race (i.e., "African American," "Bi-Racial," "Hispanic/Latino," and "White") were comparable and there were no significant differences among median parental readiness scores between all groups, $H(3)=3.908, p=.272$. As a result, child race was not entered as a covariate in any subsequent multiple hierarchal linear regression analyses.

Guardian Relationship to Child. Guardian relationship to child had a total of 9 groups: Biological Father $(n=8)$, Biological Mother $(n=23)$, Foster Father $(n=2)$, Foster Mother $(n=$ 4), Grandfather $(n=1)$, Grandmother $(n=11)$, Uncle $(n=2)$, Aunt $(n=3)$, and Other $(n=4)$. Parental readiness for change was not normally distributed across all levels of guardian relationship to child. This was determined by computing and examining the Shapiro-Wilk test statistic (i.e., $p<.05$ indicates non-normal distribution). A Kruskal-Wallis H test was computed as a non-parametric alternative to a one-way ANOVA to determine if there was a significant difference in parental readiness for change scores across guardian relationship to child groups. 
The results of the Kruskal-Wallis $\mathrm{H}$ test suggested the distributions of parental readiness scores across levels of guardian's relationship to child were comparable and there were no significant differences among median parental readiness scores between all groups, $H(8)=8.510, p=.385$. As a result, guardian relationship to child was not entered as a covariate in any subsequent multiple hierarchal linear regression analyses.

Guardian Years of Formal Education. Guardian years of formal education had a total of seven groups: Middle School $(n=3)$, High School $(n=19)$, Trade School $(n=5)$, Undergraduate college education $(n=11)$, Master's program in college $(n=5)$, Doctoral program in college $(n=3)$, and Other $(n=12)$. Parental readiness for change was not normally distributed across all levels of guardian years of formal education. This was determined by computing and examining the Shapiro-Wilk test statistic (i.e., $p<.05$ indicates non-normal distribution). A Kruskal-Wallis $\mathrm{H}$ test was computed as a non-parametric alternative to a oneway ANOVA to determine if there was a significant difference in parental readiness for change scores across guardian years of formal education groups. The results of the Kruskal-Wallis H test suggested the distributions of parental readiness scores across levels of guardian years of formal education (i.e., "middle school," "high school," "trade school," "undergraduate college education," "master's program in college," "doctoral program in college," and “other”) were comparable and there were no significant differences among median parental readiness scores between all groups, $H(6)=7.021, p=.319$. As a result, guardian years of formal education was not entered as a covariate in any subsequent multiple hierarchal linear regression analyses.

Type of Allegation. Type of allegation had a total of six groups: Witness to Domestic Violence $(n=1)$, Sexual Exploitation $(n=1)$, Drug Exposure $(n=11)$, Domestic and/or Family 
Violence $(n=1)$, Child Sexual Abuse/Assault $(n=17)$, and Child Physical Abuse $(n=3)$. Normality data could not be computed for parental readiness for change on all levels of type of allegation due to low participant count across all groups. Some children had multiple allegations and many participants were missing data. Missing data and children that fell into multiple categories were filtered out for this analysis. A Kruskal-Wallis $\mathrm{H}$ test was computed as a nonparametric alternative to a one-way ANOVA to determine if there was a significant difference in parental readiness for change scores across allegation type groups. The results of the KruskalWallis $\mathrm{H}$ test suggested the distributions of parental readiness scores across levels of allegation type were comparable and there were no significant differences among median parental readiness scores between all groups, $H(5)=7.76, p=.170$. As a result, type of allegation was not entered as a covariate in any subsequent multiple hierarchal linear regression analyses.

Guardian Race. Guardian race had a total of four groups: African American $(n=1)$, BiRacial $(n=3)$, Hispanic/Latino $(n=1)$, and White $(n=53)$. Normality data could not be computed for parental readiness for change on all levels of guardian race because the "African American" and Hispanic/Latino" categories only had one participant each. A Kruskal-Wallis H test was computed as a non-parametric alternative to a one-way ANOVA to determine if there was a significant difference in parental readiness for change scores across guardian race groups. The results of the Kruskal-Wallis $\mathrm{H}$ test suggested the distributions of parental readiness scores across levels of guardian race were comparable and there were no significant differences among median parental readiness scores between all groups, $H(3)=5.224, p=.156$. As a result, guardian race was not entered as a covariate in any subsequent multiple hierarchal linear regression analyses. 
Household Income. A one-way ANOVA was computed to determine if there was a significant difference in parental readiness for change scores across household income groups. The Shapiro-Wilk test statistic was computed to determine if parental readiness for change was normally distributed across all levels of household income. The results showed no significant deviations from normality (i.e., $p s>.05)$. Household income had a total of 7 groups: Less than $\$ 10,000(n=9), \$ 10,000-\$ 19,000(n=10), \$ 20,000-\$ 29,000(n=7), \$ 30,000-\$ 39,000(n=6)$, $\$ 40,000-\$ 49,000(n=6), \$ 50,000-\$ 59,000(n=7)$, and $\$ 60,000$ or Greater $(n=11)$. Examination of a boxplot revealed four outliers in the data, which were filtered out prior to the analysis. Levene's test for equality of variances indicated that the homogeneity of variances for parental readiness for change across levels of household income was violated $(p=.021)$. Because the data did not meet this assumption, the Welch statistic was used to determine if there were significant differences between groups in parental readiness between household income groups. The result of the one-way ANOVA analysis showed that there were no significant differences in readiness for change scores across levels of household income, Welch's $F(6,19.636)=1.181, p=.356$. As a result, household income was not entered as a covariate in any subsequent multiple hierarchal linear regression analyses.

Guardian Marital Status. A one-way ANOVA was computed to determine if there was a significant difference in parental readiness for change scores across guardian marital status groups. The Shapiro-Wilk test statistic was computed and examined to determine if parental readiness for change was normally distributed across all levels of guardian marital status. The results showed no significant deviations from normality (i.e., $p s>.05$ ). Guardian marital status had a total of 5 groups: Single $(n=17)$, Married $(n=17)$, Divorced $(n=16)$, Separated $(n=5)$, 
and Other $(n=5)$. Examination of a boxplot revealed three outliers in the data, which were filtered out prior to the analysis. Levene's test for equality of variances indicated that the homogeneity of variances for parental readiness for change across levels of guardian marital status was violated $(p=.036)$. Because the data did not meet this assumption, the Welch statistic was used to determine if there were significant differences between groups in parental readiness between guardian marital status groups. The result of the one-way ANOVA analysis showed that there were no significant differences in readiness for change scores across levels of guardian marital status, Welch's $F(4,18.854)=1.534, p=.233$. As a result, guardian marital status was not entered as a covariate in any subsequent multiple hierarchal linear regression analyses.

\section{Primary Analyses}

Hypothesis One. This hypothesis precited that there will be a significant, positive correlation between internalizing child mental health problems and parental readiness for change. To examine this hypothesis, a Pearson's bivariate correlational analysis was used. The data was examined prior to analysis to determine if a linear relationship exists between internalizing child mental health problems and parental readiness for change, potential outliers were identified, and the data was examined to determine if these variables are approximately normally distributed. Visual examination of a scatter plot suggested that a linear relationship exists between internalizing child mental health problems and parental readiness for change. Next, visual examination of a scatter plot suggested one outlier was present. This data point did not appear to be consistent with the overall data trend and was filtered out prior to analysis. To test for normality, the Shapiro-Wilk test was computed. These results showed that both internalizing 
child mental health problems and parental readiness for change are approximately normally distributed (both $p s>.05$ ). A Pearson's bivariate correlation was computed. The result supported hypothesis one and showed a significant positive relationship between internalizing child mental health problems and parental readiness for change $(r=.295, p=.019)$. This suggests that internalizing child mental health problems explained approximately $9 \%$ of the variation in parental readiness for change scores.

Hypothesis Two. Hypothesis two predicted that there will be significant, positive correlation between externalizing child mental health problems and parental readiness for change. The data was examined prior to analysis to determine if a linear relationship exists between externalizing child mental health problems and parental readiness for change, potential outliers were identified, and the data was examined to determine if these variables are approximately normally distributed. Visual examination of a scatter plot suggested that a linear relationship exists between externalizing child mental health problems and parental readiness for change. Next, visual examination of a scatter plot suggested one outlier was present. This data point did not appear to be consistent with the overall data trend and was filtered out prior to analysis. To test for normality, the Shapiro-Wilk test was computed. These results showed that parental readiness for change was approximately normally distributed $(p=.347)$. However, externalizing child mental health problems was not normally distributed $(p=.034)$. As a result, a Spearman's correlation was computed (Laerd Statistics, 2018; Privitera, 2015). The result supported hypothesis two and showed a significant, positive relationship between externalizing child mental health problems and parental readiness for change, $r_{s}(64)=.356, p=.004$, which accounted for approximately $12.7 \%$ of the variance. 
Hypothesis Three. Hypothesis three predicted that there will be significant, positive correlation between parenting stress and parental readiness for change. To examine this hypothesis, a Pearson's bivariate correlational analysis was computed. The data was examined prior to analysis to determine if a linear relationship exists between parenting stress and parental readiness for change, potential outliers were identified, and the data was examined to determine if these variables are approximately normally distributed. Visual examination of a scatter plot suggested that a linear relationship exists between parenting stress and parental readiness for change. Next, visual examination of a scatter plot suggested three outliers were present. These data points did not appear to be consistent with the overall data trend and were filtered out prior to analysis. To test for normality, the Shapiro-Wilk test was computed. These results showed that both parenting stress and parental readiness for change are approximately normally distributed (both $p s>.05$ ). A Pearson's bivariate correlation was computed. The result supported hypothesis three and showed a significant positive relationship between parenting stress and parental readiness for change $(r=.422, p=.001)$. This suggests that parenting stress explained approximately $18 \%$ of the variation in parental readiness for change scores.

Hypothesis Four. Hypothesis four predicted that the relationship between internalizing child mental health problems and parental readiness for change will be moderated by parenting stress. To examine this hypothesis, a multiple hierarchal linear regression was used. Prior to analysis, a standardized parenting stress variable was creating by summing the three parenting stress scales using their $t$-scores. Next, “dummy" variables were created for high (i.e., percentile rank 85 and above) and low (percentile rank 84 and below) parenting stress. In the current study, 59 participants fell in the "low" stress category (92.2\% of the sample) and 5 participants fell in 
the "high" stress category ( $7.8 \%$ of the sample). The percentile rank of 85 and above/below was used in order to demark between typical parenting stress levels and "borderline" or "high" levels of parenting stress that are clinically relevant. This score is consistent for "borderline" or "high" levels of parenting stress on the PSI-4 and SIPA. Then a centered variable for Internalizing problems was computed by transforming the variable (i.e., subtracting the overall mean score from all participant scores). Next, interaction term variables were created for both low and high parenting stress (i.e., low stress $\mathrm{x}$ internalizing problems-centered and high stress $\mathrm{x}$ internalizing problems-centered). In Model one, child age and open FCC case status were entered as covariates. In Model two, Internalizing Problems-Centered and Parenting Stress (dummy variable) were regressed onto the criterion variable, Parental Readiness for Change. Next, the interaction term (Internalizing Problems-Centered x Parenting Stress-Dummy) was entered into Model three. Collinearity diagnostics and the covariance matrix were included in the output. Furthermore, unstandardized predicted values, studentized and studentized deleted residuals, Cook's distance, and Leverage values were saved as new variables during the first run of the analysis. To address the assumptions of this analysis, a scatterplot was created and examined visually to provide support for a linear relationship. Homoscedasticity did not appear to be violated by visual examination of a scatter plot of the studentized residuals and unstandardized predicted values grouped by high/low stress (Draper \& Smith, 1998; Huitema, 2011; Kutner et al., 2005; Laerd Statistics, 2015b; Weisberg, 2014). Normality was tested by computing the Shapiro-Wilk statistic for the studentized residuals. The results of this analysis indicated that the data was approximately normally distributed $(p=.414)$ Next, multicollinearity was tested by examining the Variance inflation factor (VIF) values in the model output. VIF values over 10 
indicate that this assumption may have been violated (Cohen, et al., 2003). In the current analysis, the VIF value for Internalizing-Centered was 47.77 and the interaction term VIF was 40.25. Following this, outliers, leverage values, and influential cases were assessed. No outliers were identified by identifying studentized deleted above or below three standard deviations (Cohen et al., 2003). Four leverage points were identified by comparing the leverage values to the computed leverage value cutoff .28125 using the formula $3 \mathrm{p} / \mathrm{n}$, where $p=$ number of parameters plus intercept and $n=$ number of participants (Cohen et al., 2003; Kutner et al., 2005). Additionally, no influential cases were identified using Cook’s distance (Cook \& Weisberg, 1982). After these cases were filtered out and the analysis was rerun, Model three could not be computed because there was only one participant left in the high stress category. As a result, this analysis could not be properly conducted or interpreted.

Hypothesis Five. Hypothesis five predicted that the relationship between externalizing child behavior problems and parental readiness for change will be moderated by parenting stress. To examine this hypothesis, multiple hierarchal linear regression was used. The same procedure that was used for Hypothesis four was applied to Hypothesis five, except that the Externalizing Problems predictor variable was used in place of Internalizing Problems. Therefore, only modifications to the existing procedure for the current analysis are detailed below. A centered variable for Externalizing problems was computed by transforming the variable (i.e., subtracting the overall mean score from all participant scores). Next interaction term variables were created for both low and high parenting stress (i.e., low stress x externalizing problems-centered and high stress x externalizing problems-centered). In Model one, child age and FCC case status were entered as covariates. In Model two Externalizing Problems-Centered and Parenting Stress 
(dummy variable) were regressed onto the criterion variable, Parental Readiness for Change. Next, the interaction term (Externalizing Problems-Centered x Parenting Stress-Dummy) was entered into Model three. To address the assumptions of this analysis, a scatter plot was created and examined visually to provide support for a linear relationship. Homoscedasticity did not appear to be violated by visual examination of a scatter plot of the studentized residuals and unstandardized predicted values grouped by high/low stress (Draper \& Smith, 1998; Huitema, 2011; Kutner et al., 2005; Laerd Statistics, 2015b; Weisberg, 2014). Normality was tested by computing the Shapiro-Wilk statistic for the studentized residuals. The results of this analysis indicated that the data was approximately normally distributed $(p=.744)$ Next, multicollinearity was tested by examining the Variance inflation factor (VIF) values in the model output. VIF values over 10 indicate that this assumption may have been violated (Cohen, et al., 2003). In the current study, the VIF value for externalizing problems was 18.44 and the interaction term VIF was 15.09. Following this, outliers, leverage values, and influential cases were assessed. No outliers were identified by identifying studentized deleted above or below three standard deviations (Cohen et al., 2003). Three leverage points were identified by comparing the leverage values to the computed leverage value cutoff .28125 using the formula $3 \mathrm{p} / \mathrm{n}$, where $p=$ number of parameters plus intercept and $n=$ number of participants (Cohen et al., 2003; Kutner et al., 2005). Additionally, no influential cases were identified using Cook's distance (Cook \& Weisberg, 1982). After these cases were filtered out and the analysis was rerun, the VIF value for Externalizing-Centered jumped to 182.57 and the VIF value for the interaction term was 165.75. The analysis could not be conducted due to violation of multicollinearity. 
Main Effects. Hypotheses four and five were unable to be conducted due to violation of multicollinearity and/or issues regarding small number of participants in the high stress category. As a result, the interaction term was dropped from the model. The dichotomous parenting stress variable was substituted with the continuous version of the parenting stress variable low participant count in the high stress category. In Model one, child age and FCC status were entered as covariates. In Model two Internalizing Problems-Centered, Externalizing ProblemsCentered, and Parenting Stress (continuous variable) were regressed onto the criterion variable, Parental Readiness for Change. Collinearity diagnostics and the covariance matrix were included in the output. Furthermore, unstandardized predicted values, studentized and studentized deleted residuals, Cook's distance, and Leverage values were saved as new variables during the first run of the analysis. To address the assumptions of this analysis, scatter plots were created and examined visually and provided support for a linear relationship. Homoscedasticity did not appear to be violated by visual examination of a scatter plot of the studentized residuals and unstandardized predicted values (Draper \& Smith, 1998; Huitema, 2011; Kutner et al., 2005; Laerd Statistics, 2015b; Weisberg, 2014). Normality was tested by computing the Shapiro-Wilk statistic for the studentized residuals. The results of this analysis indicated that the data was approximately normally distributed $(p=.542)$. Next, multicollinearity was tested by examining the Variance inflation factor (VIF) values in the model output. VIF values over 10 indicate that this assumption may have been violated (Cohen, et al., 2003). For this analysis, the VIF values ranged from 1.047 to 2.29 , indicating that the assumption of multicollinearity was not violated. Following this, outliers, leverage values, and influential cases were assessed. One outlier was identified by identifying studentized deleted above or below three standard deviations (Cohen et 
al., 2003) and filtered out prior to analysis. No leverage points were identified by comparing the leverage values to the computed leverage value cutoff .28125 using the formula $3 \mathrm{p} / \mathrm{n}$, where $p=$ number of parameters plus intercept and $n=$ number of participants (Cohen et al., 2003; Kutner et al., 2005). Additionally, no influential cases were identified using Cook's distance (Cook \& Weisberg, 1982).

Results of the main effects analysis revealed that after controlling for child age and FCC status, there was not a significant linear relationship between parenting stress $(\beta=.131, S E=$ $.065, p=.374)$ and parental readiness for a change. Similarly, both internalizing problems $(\beta=$ $.211, S E=.119, p=.139)$, and externalizing problems $(\beta=.078, S E=.141, p=.634)$ did not show a significant linear relationship with parental readiness for change (See Table 3 below). In model one, Open FCC status did not significantly predict readiness for change $(\beta=.170, S E=$ $2.60, p=.154)$. In the end, Child age remained as the only tested variable to significantly predict parental readiness for change $(\beta=-.390, S E=.352, p=.002$; See Table 3 below $)$.

\section{Table 3}

Multiple Regression Predicting Parenting Stress From Child Age, Family Court Case Status, Internalizing problems, Externalizing problems, and Parenting Stress

\begin{tabular}{|c|c|c|c|c|}
\hline \multirow[b]{3}{*}{ Variable } & \multicolumn{4}{|c|}{ Parental Readiness for Change } \\
\hline & \multicolumn{2}{|c|}{ Model 1} & \multicolumn{2}{|c|}{ Model 2} \\
\hline & $\beta$ & $t$ & $\beta$ & $t$ \\
\hline Constant & & $10.15^{* *}$ & & $6.90 * *$ \\
\hline Child Age & -.39 & $-3.32 *$ & -.37 & $-3.15 *$ \\
\hline Open Family Court Case & .17 & 1.44 & .21 & 1.88 \\
\hline Internalizing Problems & & & .21 & 1.45 \\
\hline Externalizing Problems & & & .08 & .48 \\
\hline Parenting Stress & & & .13 & .90 \\
\hline
\end{tabular}

Note. $N=64 . * p<.05, * * p<.001$. Reproduced in Appendix F. 
Exploratory Analyses. An exploratory analysis using hierarchal linear regression was conducted to examine whether externalizing problems predicted parental stress over and above internalizing problems. Preliminary analyses were conducted to determine if any study demographic variables were statistically related to the outcome variable, parenting stress. Overall, none of the study demographics were significantly related to parenting stress. Specifically, A Pearson's bivariate correlation was computed and showed no significant relationship between guardian age and parenting stress $(r=-.513, p=.226)$. Similarly, a Spearman's correlation was computed and showed no significant relationship between child age and parenting stress, $r_{s}(64)=-.024, p=.849$. There was also no significant difference in parenting stress when male and female children were compared, $t(62)=-373, p=.710$. Furthermore, there was no significant difference in parenting stress when male and female guardians were compared, $t(62)=.205, p=.838$. Additionally, there was no significant difference in parenting stress when open CPS and no open CPS case groups were compared, $t(62)=-.317, p=.772$. Similarly, there was no significant difference in parenting stress when FCC and no FCC case groups were compared, $t(62)=1.288, p=.202$. Normality data for child race could not be computed for parenting stress due to insufficient number of participants in all categories. A Kruskal-Wallis H test was computed as a non-parametric alternative to a one-way Analysis of Variance (ANOVA) and showed there were no significant differences parenting stress scores among child race groups, $H(3)=4.211, p=.240$. The same problem occurred when comparing guardian race groups in relation to parenting stress. Therefore A Kruskal-Wallis H test was computed as a non-parametric alternative to a one-way Analysis of Variance (ANOVA) and showed there were no significant differences in parenting stress scores among guardian race 
groups, $H(3)=.888, p=.828$. A one-way ANOVA showed that there were no significant differences in parenting stress between guardian years of education groups $F(6,54)=.168, p=$ 984. A one-way ANOVA showed that there were no significant differences in parenting stress between guardian marital status groups $F(4,56)=1.560, p=.198$ (two outliers were removed prior to analysis after examination of a boxplot). A one-way ANOVA showed that there were no significant differences in parenting stress between household income groups $F(6,48)=1.815, p$ $=.116$ (five outliers were removed prior to analysis after examination of a boxplot). A KruskalWallis $\mathrm{H}$ test showed that the distributions of parenting stress scores across levels of allegation type were comparable and there were no significant differences among median parental stress scores between groups, $H(5)=3.76, p=.584$. Lastly, normality data for guardian relationship to child could not be computed due to insufficient number of participants in all categories. A Kruskal-Wallis $\mathrm{H}$ test was computed as a non-parametric alternative to a one-way Analysis of Variance (ANOVA) and showed there were no significant differences in parenting stress scores among guardian relationship to child groups, $H(8)=4.170, p=.841$. As a result, no covariates were entered into the following analysis.

In Model one, Internalizing Problems (Standardized $t$-score) was regressed onto Parenting Stress (continuous variable). In Model two, Externalizing Problems (Standardized $t$ score) was regressed onto Parenting Stress (continuous variable). Unstandardized predicted values, studentized and studentized deleted residuals, Cook's distance, and Leverage values were saved as new variables during the first run of the analysis. To address the assumptions of this analysis, scatter plots were created and examined visually and provided support for linear relationships. Homoscedasticity did not appear to be violated by visual examination of a scatter 
plot of the studentized residuals and unstandardized predicted values (Draper \& Smith, 1998; Huitema, 2011; Kutner et al., 2005; Laerd Statistics, 2015b; Weisberg, 2014). Normality was tested by computing the Shapiro-Wilk statistic for the studentized residuals. The results of this analysis indicated that the data is approximately normally distributed $(p=.077)$. Next, multicollinearity was tested by examining the Variance inflation factor (VIF) values in the model output. VIF values over 10 indicate that this assumption may have been violated (Cohen, et al., 2003). For this analysis, the VIF values ranged from 1.00 to 1.529 , indicating that the assumption of multicollinearity was not violated. Following this, outliers, leverage values, and influential cases were assessed. One outlier was identified by identifying studentized residuals above or below three standard deviations (Cohen et al., 2003) and filtered out prior to analysis. No leverage points were identified by comparing the leverage values to the computed leverage value cutoff 0.2 (Huber, 1981). Also, no influential cases were identified using Cook's distance (Cook \& Weisberg, 1982), and the Durbin-Watson statistic was 1.867 , indicating that there was independence of observations (Draper \& Smith, 1998; Laerd Statistics, 2015a).

Results of this analysis showed that internalizing problems alone significantly predicted parenting stress, $R^{2}=.313, F(1,61)=27.828, p<.001 ;$ adjusted $R^{2}=.302$. After adding externalizing problems to the model, it predicted parenting stress over and above just internalizing problems, $R^{2}=.503, F(1,60)=22.871, p<.001$. See Table 3 Below for summary of results. 


\section{Table 4}

Hierarchical Multiple Regression Predicting Parenting Stress From Internalizing and Externalizing problems

\begin{tabular}{lllll}
\hline & \multicolumn{4}{c}{ Parenting Stress } \\
\cline { 2 - 5 } Variable & \multicolumn{3}{c}{ Model 1 } & Model 2 \\
\cline { 2 - 5 } Constant & $\mathrm{B}$ & $\mathrm{B}$ & $\beta$ \\
Internalizing Problems & 1.09 & .56 & .32 .86 & .22 \\
Externalizing Problems & & & 1.05 & .54 \\
& & & \\
$R^{2}$ & .313 & .503 & \\
$F$ & $27.83^{* *}$ & $22.87^{* *}$ \\
$\Delta R^{2}$ & .313 & .190 & \\
$\Delta F$ & $27.83^{* *}$ & $22.87^{* *}$ \\
\hline Note. $N=64 . * p<.05, * * p<.001$. Reproduced in Appendix G.
\end{tabular}




\section{CHAPTER 5: DISCUSSION}

\section{Review of Current Study}

The current study applied the Transtheoretical Model of behavior change (TTM;

Prochaska \& DiClemente, 1983) to parenting behavior, as past research has suggested that increasing parental motivation and readiness for change is associated with greater client participation and improved therapeutic outcomes (Dowell \& Ogles, 2010; Karver et al., 2006). More specifically, the current study attempted to replicate Jones et al.'s (2017) primary findings and add to the current body of literature on parental readiness for change by examining parenting stress as a moderator of the relationship between internalizing and externalizing child problems and parental readiness for change (Jones et al., 2017; Wade \& Andrade, 2015). I also set out to investigate the "threshold" model explained by Jones et al. (2017), which suggested that there is a "...level of parenting stress at which it no longer matters whether the child has behavior problems and the parent is ready to change regardless of child behavior..." (p. 227). In line with this model, Jones et al. (2017) found that when parenting stress was high, parental readiness for change was also high at both low and high levels of child externalizing behavior problems. This study's findings add to the few primary examples that have investigated parental readiness for change as a clinical variable (e.g., Jones et al., 2017; Littell \& Girvin, 2005; Wade \& Andrade, 2015).

\section{Review of Findings}

Readiness for Change and Demographic Variables. The current study found that two demographic variables emerged as significantly related to parental readiness for change. Namely, 
data suggested that there was a significant negative relationship between parental readiness for change and child age; thus, younger child age predicted higher levels of parental readiness for change among guardians. The second finding showed that there was a significant difference in parental readiness for change when comparing family court case (FFC) status groups. Specifically, open FCC showed significantly higher levels of readiness for change in comparison to the no FFC group. This means that guardians who reported that they were currently involved with family court also tended to report higher levels of parental readiness for change.

Hypotheses One and Two. Hypothesis one predicted a significant, positive relationship between internalizing child mental health problems and parental readiness for change. A Pearson's bivariate correlation was computed and showed a significant positive relationship between internalizing child mental health problems and parental readiness for change and explained a small amount of variance in parental readiness for change scores. Hypothesis two predicted that there would be a significant positive relationship between externalizing child mental health problems and parental readiness for change. Results showed a significant, positive relationship between externalizing child mental health problems and parental readiness for change, which means that guardians who endorsed higher intensity of externalizing behavior problems (e.g., oppositional behaviors and hyperactivity) also tended to report higher levels of readiness to change parenting behaviors.

These results are consistent with existing research. The relationship between internalizing child mental health problems and parental readiness has been previously supported. In particular, Wade and Andrade's (2015) study investigating the factor structure of the Parent Readiness for Change Scale (PRFCS) showed that readiness scores were significantly related to childhood 
emotional problems in addition to childhood conduct problems. Following this study, Jones, et al. (2017) also found significant positive relationships between all three clinical variables: hyperactivity/impulsivity symptoms, oppositional symptoms and parental readiness for change. Similarly, Andrade and colleagues (2015) found that parents who reported more disruptive behavior problems were also more likely to report that they are ready for change. Lastly, a validation study for a parental readiness for change measure (i.e., Readiness, Efficacy, Attributions, Defensiveness, and Importance Scale - Short Form; READI-SF) found evidence that child behavior problems explained $24 \%$ of the variance in reported overall parental readiness for change (Proctor et al., 2017). However, as I will address later in the discussion, internalizing and externalizing problems were not found to have any predictive power on readiness for change after controlling for child age and FCC status.

Hypothesis Three. Hypothesis three predicted a significant, positive relationship between parenting stress and parental readiness for change. Results supported this hypothesis and showed a significant positive relationship between parenting stress and parental readiness for change. These findings are consistent with Jones et al. (2017), who also found a significant positive relationship between hyperactivity/impulsivity symptoms, oppositional symptoms and parenting stress. However, as I will discuss in the following section, parenting stress was not found to have any predictive power on readiness for change after controlling for child age and FCC status.

Hypotheses Four \& Five. Hypothesis four predicted that the relationship between internalizing child mental health problems and parental readiness for change will be moderated 
by parenting stress. This analysis could not be conducted due to violation of multicollinearity and an inability to validly interpret the findings.

Hypothesis five predicted that the relationship between externalizing child behavior problems and parental readiness for change will be moderated by parenting stress. After outliers and leverage points were filtered out, the analysis could not be examined because there was only one participant remaining in the high stress group. As a result, this analysis was ended because it could not be conducted or be properly interpreted. The study by Jones et al. (2017) served as a model for the current research investigation. Their findings showed that parenting stress significantly moderated the relationship between hyperactivity/impulsivity symptoms and parental readiness for change. The researchers also noted that this interaction effect was present for child oppositional symptoms as well. Furthermore, they noted that the relationship between parenting stress and both of these clinical variables were significant (e.g., hyperactivity/impulsivity symptoms, $r=.37, p<001$; oppositional symptoms, $r=.52, p<001$ ). The multicollinearity assumption is not mentioned and VIF and tolerance scores are not reported. They also reported that preliminary analyses were performed to test the relationships between demographic variables and clinical variables. They reported that two covariates were added to the model as control variables (i.e., whether the child was taking medication and whether they had a previous ADHD diagnosis). In the current study, multicollinearity as indicated by variance inflation factor (VIF) levels well over 10 prevented the examination of the of the moderation analyses in the current study. However, the current study did replicate and show significant positive relationships between externalizing problems, parenting stress, and parental readiness for change. 
Main Effects Analysis. Analyses for hypotheses four and five were unable to be conducted due to the violation of multicollinearity and/or issues regarding the small number of participants in the high stress category. As a result, the interaction term was dropped from the model and the main effects model was investigated. In Model one, child age was entered as a covariate. In Model two, Internalizing Problems-Centered, Externalizing Problems-Centered, and Parenting Stress (continuous variable) were regressed onto the criterion variable, Parental Readiness for Change. The findings of the main effects analysis showed that after controlling for child age and FCC status, there was not a significant linear relationship between parenting stress and parental readiness for a change. Additionally, both internalizing problems and externalizing problems did not significantly predict parental readiness for change. When put head-to-head in Model one of the main effects analysis, FCC status did not significantly predict readiness for change. However, child age did significantly predict parental readiness for change. In review, the current study found that after controlling for child age and FCC status, child internalizing problems, externalizing problems, and parenting stress did not predict level of parental readiness for change.

These results indicate that guardians presenting for therapy with younger children also reported greater levels of parental readiness for change in comparison with guardians with older children. One line of inquiry may ask if child age is correlated with problem severity and parent stress, accounting for the relationship between child age and readiness. However, child age was not significantly correlated with internalizing problems, externalizing problems, or parenting stress. Therefore, it appears that out of all the tested variables in this study, child age accounts for the largest share of the variance in parental readiness for change. 
A child's age may in fact serve as an important variable when caregivers consider making changes in their own behavior. One analog to this finding was illustrated in a study on parental readiness to change behaviors that may help their child lose weight (Rhee et al., 2005). Specifically, Rhee and colleagues (2005) found that age (8 years or older), parents believing that they themselves were overweight, and believing that their child's weight posed a health concern, were the variables associated with parental readiness to make changes in their child's diet. The authors indicate that health issues associated with weight become harder to address as children age because younger children may be "more amenable to change" (p. e98). A similar sentiment may also be driving higher levels of parental readiness for change with younger children with the current study's clinical population. This interpretation connects child age to the individual change process posited by the TTM (DiClemente, \& Prochaska, 1982; Prochaska, \& DiClemente, 1982, 1983; Prochaska et al., 1985). This model was used as an explanatory model to understand the relationship between child behavior problems and parental readiness for change regarding their parenting behaviors for children and families seeking mental health treatment (e.g., Jones et al., 2017; Proctor et al., 2017; Wade \& Andrade 2015). While it may be true that in some cases involving the need for parenting changes to occur, "Children cannot wait" (Corden \& Somerton, 2004, p. 1041). However, for ineffective, but not abusive discipline strategies, parents may be undergoing a change process in parenting when they engage in therapeutic services with their child. For instance, during parent-child interaction therapy (PCIT) guardians learn and implement new ways of interacting with their child (Child Welfare Information Gateway, 2019). 
An alternative explanation may suggest the potential impact of a "third variable." For instance, perhaps type of intervention could explain the predictive influence that age has on parental readiness for change. For instance, younger children and guardians that present for therapy at the clinic may expect or may even be informed that they will participate in a parentchild interaction (e.g., PCIT or Dyadic therapy). This intervention places the guardian in an active role as they learn and implement specialized skills one-on-one with their child (Child Welfare Information Gateway, 2019). As a result, the effectiveness of this intervention may be highly impacted by the guardian's level of treatment engagement and motivation to participate. As such, some researchers have even worked to develop modified PCIT interventions to reduce drop-out and increase engagement among guardians that plan to participate in this type of intervention (e.g., Chaffin et al., 2009; Webb et al., 2017). The Jones et al. (2017) study is the most similar analog to the current study. However, their results did not suggest any impact of client age on parental readiness for change. The authors noted that the study took place at an ADHD Assessment Clinic at a children's hospital. If the focus of the clinic was assessment based, it is possible that intervention type was decoupled from the data collection process. Also, it is possible that the clinical population was more homogenous in nature. On the other hand, participants for the current study may have had expectations for treatment in mind as they were completing the intake documents, which could have influenced their responses on the parental readiness for change measure.

Exploratory Analysis. An exploratory analysis was conducted after the primary analyses had concluded. This analysis investigated whether externalizing behavior problems predicted parent stress over and above internalizing problems. In Model one, Internalizing Problems 
(Standardized $t$-score) was regressed onto Parenting Stress (continuous variable). In Model two, Externalizing Problems (Standardized $t$-score) was regressed onto Parenting Stress (continuous variable). The results showed that internalizing problems significantly predicted the level of parenting stress. However, the model predicted parenting stress over and above just internalizing problems once externalizing problems was added.

Existing research on parenting stress and externalizing child behavior problems has suggested that they are bidirectionally related and develop together over time, which is consistent with the transactional model of development (Barry et al., 2005; Deater-Deckard, 1998; Neece et al., 2012; Rodriguez, 2011; Sameroff, 1975; Stone et al., 2016). Stone and colleagues (2016) conducted a crossed-lagged path analysis to examine the development of the relationship between internalizing problems, externalizing problems and parenting stress over time. Their study found that over time, parenting stress decreased as externalizing problems decreased. They did not find the same relationship among internalizing problems. The current study found that externalizing problems predicted parenting stress over and above just internalizing problems. Taken together, these results may indicate that externalizing problems have a stronger impact on parenting stress than internalizing problems.

\section{Limitations}

The data for current study was collected during the 2020 COVID-19 global pandemic. As a result, a portion of participants in this study have been characterized by a unique set of life circumstances. Therefore, it is unclear if the results of this study will generalize to similar clinical situations in the future. Furthermore, the current study was conducted with a clinical 
population characterized by childhood abuse and neglect. As a result, these findings may be specific to these clinical populations and my not generalize to other clinical populations or age groups. Additionally, most respondents in the study were majority White (and Female). For these reasons, the results of this study may lack generalizability outside of these demographic groups (e.g., populations that identify as Black, Indigenous, or People of Color).

The current study also used a correlational design. Due to this, it is not appropriate to draw causal conclusions from the data and the study findings. For instance, age may have no causal impact on parental readiness for change. The study was also cross-sectional, meaning that it cannot be determined if higher levels of readiness for change led to greater levels of guardian involvement in treatment or lower therapy attrition levels. However, past research may suggest that greater pre-treatment readiness does boost guardian involvement in treatment (Andrade et al., 2015). The ability to adequately examine these questions may help clinicians better understand the connection between parental readiness for change, treatment outcomes and measure the impact that this may have on attrition rates. In the future, gathering data on client and guardian engagement (e.g., attendance), guardian mastery of parenting skills taught in therapy, and symptom tracking over time may help researchers and clinicians better understand the relationships between parental readiness for change and real examples of the change process in therapy. One way that this may be achieved is with the use of a longitudinal cross-lagged regression approach. This would allow researchers to gather data on readiness and other clinical variables at multiple time-points, which could help researchers draw more meaningful conclusions on the relationships between parental readiness for change and other clinical variables over time (Kearney, 2017). 
Multicollinearity driven by the strong associations between child mental health problem severity and parenting stress prevented the examination of the of the moderation analyses in the current study. Moreover, the results showed that both internalizing and externalizing problems were highly correlated with parenting stress. The results suggest that internalizing problems alone accounted for about $25 \%$ of the variation in parenting stress scores. Mean-centering predictor variables is one approach to reducing multicollinearity, however, as some researchers argue, this approach may not alleviate multicollinearity that exists on a "macro" level (Iacobucci et al., 2016).

Another limitation for the current study is that internal consistency reliability estimates (Cronbach's alpha values) could not be computed for the measures in the current sample. The clinical database only contained total scores and did not include individual item responses. Clinical data for the study was gathered during new client intakes and standardized scores were computed using scoring software (e.g., PARiConnect ${ }^{\mathrm{TM}}$ and Q-global ${ }^{\mathrm{TM}}$ ). The Parental Readines for Change Scale - Short Form (PRFCS-SF) was hand-scored, transformed and then the total readiness score was entered into the encrypted database. As a result, internal consistency reliability could not be computed using the data available.

Perhaps one of the most critical limitations of the study is that it lacked power due to small sample size $(n=64)$ even though data collection occurred over a 24 -month period. The participant sample was suppressed due to several factors. Most notable was that the data collection took place in person and overlapped with the COVID-19 pandemic. Due to shutdowns related to the spread of the COVID-19 virus, the number of new clients was greatly decreased. Furthermore, each family admitted could only count as one new participant to avoid issues 
related to independence of observations between children in one family. In addition, my study was a replication and expansion of a study by Jones et al. (2017). My sample size $(n=64)$ appears comparable to the sample size $(n=69)$ in the Jones et al. (2017) study. Despite all this, the study and results retain a limitation due to lack of statistical power. Therefore, the results of this study should be understood within this context.

\section{Directions for Future Research}

The current study does not include a measurement of positive impression management. If it is suspected that guardians had any motivation to "fake-good" in regard to their readiness for change upon entering therapy, using a measure such as the Marlowe-Crowne Social Desirability Scale (Crowne \& Marlowe, 1960) may help uncover potential effects. One study by Zemore (2012) did examine the relationship between stage of change and social desirability for a population of participants receiving outpatient treatment for substance use issues. In that study, the author noted that individuals higher in social desirability tended to endorse more precontemplative items regarding their treatment and less preparation or change-oriented items. They concluded that high levels of social desirability led to participants downplaying the severity of their drug use issues. Despite leading to lower levels of readiness for change, Zemore (2012) found that social desirability was also positively related to treatment attendance. Meaning that an individual who is highly motivated by social desirability may report low readiness for change but also be more likely to attend therapy. Zemore (2012) also noted that this relationship between readiness for change and social desirability did not appear when the presenting concern was anxiety (see Dozois, et al., 2004) and suggested that social stigma surrounding drug use issues 
may account for this relationship. So, it appears that the impact of social desirability is complex. Would social desirability operate in the same way regarding guardians presenting for parentchild therapy? In other words, would guardians with high levels of social desirability be motivated to present as not having much reason to address their parenting approaches? Future research could investigate any impact that positive impression management has on the way guardians approach the measure.

The question remains whether intervention type is driving readiness for parental change and acting as a "third variable" in the current study. To investigate this question, it is recommended that future researchers collect data on the intervention type and include information from clients on their expectations for treatment. Future research may also seek to investigate whether age is a conscious factor in a parent or guardian's decision to make changes to their parenting behavior. For instance, do guardians consider making changes to parenting behavior for older children less beneficial?

There is some disagreement over the application of the TTM (Prochaska \& DiClemente, 1983) to parenting behavior. While some research has applied this model to understand the relationship between presenting problem severity and readiness (e.g., Jones et al., 2017), and others have gone as far as to work on developing scales to measure parental readiness for change (e.g., Brestan et al., 1999; Wade \& Andrade, 2015), others have argued that the stage model of change lacks clinical applicability to change in parenting behavior (Corden \& Somerton, 2004). The current study used the TTM to understand parenting behavior change. However, it may be important to assess the way in which guardians experience readiness to change (or not change) their parenting behaviors. Therefore, future studies may serve an important role in examining 
this question. This may be achieved using a qualitative investigation of parental readiness for change to uncover themes related to readiness that may serve as helpful entry points for future quantitative research. Rhee and colleagues (2005) found that age was a factor associated with parental readiness to make changes in their child's diet. To further explore these findings, researchers may pose a question about how age of their child may play a role in their level of parental readiness for change. Furthermore, a subjective examination of readiness to change parenting behavior may also prove useful in clinical applications. For example, research related to identifying strategies for increasing parental involvement in their child's treatment should be conducted, which has been investigated from a quantitative perspective (e.g., Andrade et al., 2015).

\section{Implications for Counseling Psychology}

Parental readiness for change remains an important area of study due to its association with parent participation in their child's treatment (Sterrett et al., 2010; Wade \& Andrade, 2015). This concept serves a key role for child therapy interventions due to the role that guardians play in their child's treatment (Child Welfare Information Gateway, 2019; Jones et al., 2017; Dowell \& Ogles, 2010; Karver et al., 2006), and carryover of clinical progress between the clinic and the home (Karver et al., 2005). Therapy attrition rates for children and adolescents have been estimated to range between 36-44\% (de Haan et al., 2013). Focusing on ways to increase parental readiness for change may help mitigate this issue (Webb et al., 2017).

Researchers have tried to implement strategies for increasing parent participation for interventions that require parent involvement. For instance, Webb et al. (2017) applied a 
motivational component to PCIT and compared it to the unmodified version of PCIT. They found that the intervention did not reduce client dropout, and that they both were helpful in reducing parenting stress and child behavior problems. This finding alone appears to show that their intervention was not successful. Yet, they did find that higher pre-treatment parental readiness for change was associated with decreased drop-out rates. As a result, future research that focuses on identifying ways to increase parent motivation for treatment may be beneficial (Chaffin et al., 2009; Wade, \& Andrade, 2015; Webb et al., 2017).

Motivational enhancement with the use of motivational interviewing has been shown to be a helpful tool to increase client participation in therapy (Walitzer et al., 1999). Furthermore, decisional balance or weighing the pros and cons of a behavior or potential behavior change and working to promote "change talk" in clients have been used as a method for helping clients engage in the change process and has been linked to motivational interviewing (Miller \& Rollnick, 2002, 2013; Miller \& Rose, 2015;). Moreover, Miller and Rose (2015) noted that “change talk" involves helping clients identify and communicate reasons for making a change (e.g., "pros") and posits that change may be more likely to occur when these "pros" outweigh the "cons" or reasons not to change. Motivational interviewing may be applicable to guardians presenting for therapy with their child (e.g., Webb et al., 2017). This type of intervention would utilize strategies common to motivational interviewing, which include gauging readiness for change, employing decisional balance (i.e., weighing the pros and cons of current parenting behavior and making changes to parenting behavior), using open-ended questions, affirming the guardian's strengths as a parent, use of verbal reflections, and summarizing content (OARS). The clinician may also work to identify and work to promote "change talk" from the guardian. A 
parent may voice they do not think the way they are currently responding to their child is helpful. This may be an example of "change talk" because it indicates the parent may be open to learning a new strategy for responding to their child (Adapted from Adept - Alcohol and Drug Education for Prevention and Treatment, 2017; Child Welfare Information Gateway, 2019; Miller \& Rose, 2015; Miller \& Rollnick, 2002, 2013).

\section{Conclusion}

This study was unable to replicate Jones et al.'s (2017) findings that parenting stress moderates the relationship between externalizing child problems and parental readiness for change. This was due to violation of multicollinearity and an insufficient number of participants in the high stress parenting group. Furthermore, the current study found that parenting stress was associated with higher levels of parental readiness for change. However, after controlling for child age, this relationship was no longer supported. Therefore, the current study findings call into question the relationship between readiness to change and parenting stress. An exploratory analysis found that externalizing problems predicted parenting stress over and above internalizing problems. Lastly, the current study found guardians of younger children tended to report greater levels of readiness to change parenting behaviors. This finding is beneficial to the field of Counseling Psychology as it may help clinicians better conceptualize guardians' level of readiness and help clinicians meet them "where they are" with respect to their parenting behaviors. 


\section{References}

Abidin, R. R. (1990). Parenting stress index. Charlottesville, VA: Pediatric Psychology Press. https://doi.org/10.1037/t02445-000

Abidin, R. R. (1995). Parenting stress index. Odessa, FL: Psychological Assessment Resources.

Abidin, R. R. (2012). Parenting stress index, Fourth edition, Short form. Lutz, Florida: Psychological Assessment Resources.

Aiken, L. S., \& West, S. G. (1991). Multiple regression: Testing and interpreting interactions. Thousand Oaks, CA: Sage.

Alexander, P., \& Morris, E. (2008). Stages of change in batterers and their response to treatment. Violence and Victims, 23(4), 476-492. https://doi.org/10.1891/0886-

\section{$\underline{6708.23 .4 .476}$}

Alley, E. S., Velasquez, M. M., \& von Sternberg, K. (2018). Associated factors of readiness to change in young adult risky drinkers. The American Journal of Drug and Alcohol Abuse, 44(3), 348-357. https://doi.org/10.1080/00952990.2017.1363768

Anderson, S. B., \& Guthery, A. M. (2015). Mindfulness-based psychoeducation for parents of children with attention-deficit/hyperactivity disorder: An applied clinical project. Journal of Child and Adolescent Psychiatric Nursing, 28(1), 43-49.

https://doi.org/10.1111/jcap.12103 
Andrade, B. F., Browne, D. T., \& Naber, A. R. (2015). Parenting skills and parent readiness for treatment are associated with child disruptive behavior and parent participation in treatment. Behavior Therapy, 46(3), 365-378. https://doi.org/10.1016/j.beth.2015.01.004

Anthony, L. G., Anthony, B. J., Glanville, D. N., Naiman, D. Q., Waanders, C., \& Shaffer, S. (2005). The relationships between parenting stress, parenting behaviour and preschoolers' social competence and behaviour problems in the classroom. Infant and Child Development, 14(2), 133-154. https://doi.org/10.1002/icd.385

Aronen, E. T., \& Soininen, M. (2000). Childhood depressive symptoms predict psychiatric problems in young adults. The Canadian Journal of Psychiatry / La Revue Canadienne de Psychiatrie, 45(5), 465-470. https://doi.org/10.1177/070674370004500507

Bagner, D. M., Sheinkopf, S. J., Miller-Loncar, C., LaGasse, L. L., Lester, B. M., Liu, J., ... \& Das, A. (2009). The effect of parenting stress on child behavior problems in high-risk children with prenatal drug exposure. Child Psychiatry and Human Development, 40(1), 73-84. https://doi.org/10.1007/s10578-008-0109-6

Baron, R. M., \& Kenny, D. A. (1986). The moderator-mediator variable distinction in social psychological research: Conceptual, strategic, and statistical considerations. Journal of Personality and Social Psychology, 51(6), 1173-1182. https://doi.org/10.1037/0022$\underline{3514.51 .6 .1173}$

Barry, T. D., Dunlap, S. T., Cotten, S. J., Lochman, J. E., \& Wells, K. C. (2005). The influence of maternal stress and distress on disruptive behavior problems in boys. Journal of the 
American Academy of Child \& Adolescent Psychiatry, 44(3), 265-273. https://doi.org/10.1097/00004583-200503000-00011

Becker, K. D., Lee, B. R., Daleiden, E. L., Lindsey, M., Brandt, N. E., \& Chorpita, B. F. (2015). The common elements of engagement in children's mental health services: Which elements for which outcomes? Journal of Clinical Child and Adolescent Psychology, 44(1), 30-43. https://doi.org/10.1080/15374416.2013.814543

Belding, M. A., Iguchi, M. Y., \& Lamb, R. J. (1997). Stages and processes of change as predictors of drug use among methadone maintenance patients. Experimental and Clinical Psychopharmacology, 5(1), 65-73. https://doi.org/10.1037/1064-1297.5.1.65

Bloomfield, L., \& Kendall, S. (2012). Parenting self-efficacy, parenting stress and child behaviour before and after a parenting programme. Primary Health Care Research and Development, 13(4), 364-372. https://doi.org/10.1017/S1463423612000060

Boggs, S. R., Eyberg, S. M., Edwards, D. L., Rayfield, A., Jacobs, J., Bagner, D., \& Hood, K. K. (2004). Outcomes of parent-child interaction therapy: A comparison of treatment completers and study dropouts one to three years later. Child \& Family Behavior Therapy, 26(4), 1-22. https://doi.org/10.1300/J019v26n04_01

Boswell, J. F., Sauer-Zavala, S. E., Gallagher, M. W., Delgado, N. K., \& Barlow, D. H. (2012). Readiness to change as a moderator of outcome in transdiagnostic treatment. Psychotherapy Research, 22(5), 570-578.

https://doi.org/10.1080/10503307.2012.688884 
Brestan, E. V., Ondersma, S. J., Simpson, S. M., \& Gurwitch, R. (1999, April). Application of stage of change theory to parenting behavior: Validating the Parent Readiness to Change Scale. Poster presented at the Florida Conference on Child Health Psychology, Gainesville, Florida.

Carey, K. B., Purnine, D. M., Maisto, S. A., \& Carey, M. P. (1999). Assessing readiness to change substance abuse: A critical review of instruments. Clinical Psychology: Science and Practice, 6(3), 245-266. https://doi.org/10.1093/clipsy.6.3.245

Chacko, A., Wymbs, B. T., Chimiklis, A., Wymbs, F. A., \& Pelham, W. E. (2012). Evaluating a comprehensive strategy to improve engagement to group-based behavioral parent training for high-risk families of children with ADHD. Journal of Abnormal Child Psychology, 40(8), 1351-1362. https://doi.org/10.1007/s10802-012-9666-z

Chaffin, M., Valle, L. A., Funderburk, B., Gurwitch, R., Silovsky, J., Bard, D., ... \& Kees, M. (2009). A motivational intervention can improve retention in PCIT for low-motivation child welfare clients. Child Maltreatment, 14(4), 356-368. https://doi.org/10.1177/1077559509332263

Chi, P., Li, X., Tam, C. C., Du, H., Zhao, G., \& Zhao, J. (2015). Parenting mediates the impact of caregivers' distress on children's well-being in families affected by HIV/AIDS. AIDS and Behavior, 19(11), 2130-2139. https://doi.org/10.1007/s10461-015-1104-0

Child Welfare Information Gateway. (2019). Parent-child interaction therapy: A primer for child welfare professionals. Washington, DC: U.S. Department of Health and Human Services, Children's Bureau. 
Cohen, J. (1988). Statistical power analysis for the behavioral sciences (2nd ed.). Hillsdale, NJ: Lawrence Earlbaum Associates.

Cohen, J., Cohen, P., West, S. G., \& Aiken, L. S. (2003). Applied multiple regression/correlation analysis for the behavioral sciences (3rd ed.). Mahwah, NJ: Lawrence Erlbaum Associates.

Corden, J., \& Somerton, J. (2004). The trans-theoretical model of change: A reliable blueprint for assessment in work with children and families? British Journal of Social Work, 34(7), 1025-1044. https://doi.org/10.1093/bjsw/bch127

Cortina, J. M. (1993). What is coefficient alpha? An examination of theory and applications. Journal of Applied Psychology, 78(1), 98-104. https://doi.org/10.1037/0021$\underline{9010.78 .1 .98}$

Costa, N. M., Weems, C. F., Pellerin, K., \& Dalton, R. (2006). Parenting stress and childhood psychopathology: An examination of specificity to internalizing and externalizing symptoms. Journal of Psychopathology and Behavioral Assessment, 28(2), 113-122. https://doi.org/10.1007/s10862-006-7489-3

Crnic, K. A., Gaze, C., \& Hoffman, C. (2005). Cumulative parenting stress across the preschool period: Relations to maternal parenting and child behaviour at age 5. Infant and Child Development, 14(2), 117-132. https://doi.org/10.1002/icd.384

Crowne, D. P., \& Marlowe, D. (1960). A new scale of social desirability independent of psychopathology. Journal of Consulting Psychology, 24(4), 349-354. https://doi.org/10.1037/h0047358 
Crum, K. I., \& Moreland, A. D. (2017). Parental stress and children's social and behavioral outcomes: The role of abuse potential over time. Journal of Child and Family Studies, 26(11), 3067-3078. https://doi.org/10.1007/s10826-017-0822-5

Cunningham, C. E., \& Boyle, M. H. (2002). Preschoolers at risk for attention-deficit hyperactivity disorder and oppositional defiant disorder: Family, parenting, and behavioral correlates. Journal of Abnormal Child Psychology, 30(6), 555-569. https://doi.org/10.1023/A:1020855429085

Daley, A. J., \& Duda, J. L. (2006). Self-determination, stage of readiness to change for exercise, and frequency of physical activity in young people. European Journal of Sport Science, 6(4), 231-243. https://doi.org/10.1080/17461390601012637

Dawson, J. F. (2014). Moderation in management research: What, why, when, and how. Journal of Business and Psychology, 29(1), 1-19. https://doi.org/10.1007/s10869-013-9308-7

de Haan, A. M., Boon, A. E., de Jong, J. T. V. M., Hoeve, M., \& Vermeiren, R. R. J. M. (2013). A meta-analytic review on treatment dropout in child and adolescent outpatient mental health care. Clinical Psychology Review, 33(5), 698-711. https://doi.org/10.1016/j.cpr.2013.04.005

Deater-Deckard, K. (1998). Parenting stress and child adjustment: Some old hypotheses and new questions. Clinical Psychology: Science and Practice, 5(3), 314-332. https://doi.org/10.1111/j.1468-2850.1998.tb00152.x 
Delgadillo, J., \& Groom, M. (2017). Using psychoeducation and role induction to improve completion rates in cognitive behavioural therapy. Behavioural and Cognitive Psychotherapy, 45(2), 170-184. https://doi.org/10.1017/S1352465816000643

Demmel, R., Beck, B., Richter, D., \& Reker, T. (2004). Readiness to change in a clinical sample of problem drinkers: Relation to alcohol use, self-efficacy, and treatment outcome. European Addiction Research, 10(3), 133-138. https://doi.org/10.1159/000077702

DiClemente, C.C., Daniels, J.W., Bellino, L.E., \& Neavins, T.M. (1999). Readiness to change and abstinence self-efficacy in alcoholism treatment. Paper presented at the 1999 conference of the Association for the Advancement of Behavior Therapy, Toronto, Canada, November.

DiClemente, C. C., \& Hughes, S. O. (1990). Stages of change profiles in outpatient alcoholism treatment. Journal of Substance Abuse, 2(2), 217-235. https://doi.org/10.1016/S0899$\underline{3289(05) 80057-4}$

DiClemente, C. C., \& Prochaska, J. O. (1982). Self-change and therapy change of smoking behavior: A comparison of processes of change in cessation and maintenance. Addictive Behaviors, 7(2), 133-142. https://doi.org/10.1016/0306-4603(82)90038-7

DiClemente, C. C., Prochaska, J. O., \& Gibertini, M. (1985). Self-efficacy and the stages of selfchange of smoking. Cognitive Therapy and Research, 9(2), 181-200. https://doi.org/10.1007/BF01204849

DiClemente, C. C., Prochaska, J. O., Fairhurst, S. K., Velicer, W. F., Velasquez, M. M., \& Rossi, J. S. (1991). The process of smoking cessation: An analysis of precontemplation, 
contemplation, and preparation stages of change. Journal of Consulting and Clinical Psychology, 59(2), 295-304. https://doi.org/10.1037/0022-006X.59.2.295

Dowell, K. A., \& Ogles, B. M. (2010). The effects of parent participation on child psychotherapy outcome: A meta-analytic review. Journal of Clinical Child and Adolescent Psychology, 39(2), 151-162. https://doi.org/10.1080/15374410903532585

Dozois, D. J. A., Westra, H. A., Collins, K. A., Fung, T. S., \& Garry, J. K. F. (2004). Stages of change in anxiety: Psychometric properties of the University of Rhode Island Change Assessment (URICA). Behaviour Research and Therapy, 42(6), 711-729.

Draper, N. R., \& Smith, H. (1998). Applied regression analysis (3rd ed.). New York: Wiley. https://doi.org/10.1002/9781118625590

Dufour, J. M., \& Dagenais, M. G. (1985). Durbin-Watson tests for serial correlation in regressions with missing observations. Journal of Econometrics, 27(3), 371-381. https://doi.org/10.1016/0304-4076(85)90012-0

Durbin, J., \& Watson, G. S. (1951). Testing for serial correlation in least squares regression. II. Biometrika, 38(1/2), 159-177. https://doi.org/10.2307/2332325

Faul, F., Erdfelder, E., Buchner, A., \& Lang, A.G. (2009). Statistical power analyses using G*Power 3.1: Tests for correlation and regression analyses. Behavior Research Methods, 41, 1149-1160. https://doi.org/10.3758/BRM.41.4.1149

Field, A. (2013). Discovering statistics using IBM SPSS statistics. London: Sage. 
Gach, E. J., Ip, K. I., Sameroff, A. J., \& Olson, S. L. (2018). Early cumulative risk predicts externalizing behavior at age 10: The mediating role of adverse parenting. Journal of Family Psychology, 32(1), 92-102. https://doi.org/10.1037/fam0000360

Haine-Schlagel, R., \& Walsh, N. E. (2015). A review of parent participation engagement in child and family mental health treatment. Clinical Child and Family Psychology Review, 18(2), 133-150. https://doi.org/10.1007/s10567-015-0182-X

Harachi, T. W., Fleming, C. B., White, H. R., Ensminger, M. E., Abbott, R. D., Catalano, R. F., \& Haggerty, K. P. (2006). Aggressive Behavior Among Girls and Boys During Middle Childhood: Predictors and Sequelae of Trajectory Group Membership. Aggressive Behavior, 32(4), 279-293. https://doi.org/10.1002/ab.20125

Haydicky, J., Shecter, C., Wiener, J., \& Ducharme, J. M. (2015). Evaluation of MBCT for adolescents with ADHD and their parents: Impact on individual and family functioning. Journal of Child and Family Studies, 24(1), 76-94. https://doi.org/10.1007/s10826-0139815-1

Heath, C. L., Curtis, D. F., Fan, W., \& McPherson, R. (2015). The association between parenting stress, parenting self-efficacy, and the clinical significance of child ADHD symptom change following behavior therapy. Child Psychiatry and Human Development, 46(1), 118-129. https://doi.org/10.1007/s10578-014-0458-2

Henderson, M. J., Saules, K. K., \& Galen, L. W. (2004). The predictive validity of the University of Rhode Island Change Assessment Questionnaire in a heroin-addicted 
polysubstance abuse sample. Psychology of Addictive Behaviors, 18(2), 106-112. https://doi.org/10.1037/0893-164X.18.2.106

Huber, P. J. (1981). Robust statistics. New York: John Wiley \& Sons. https://doi.org/10.1002/0471725250

Huitema, B. E. (2011). The analysis of covariance and alternatives (2nd ed.). Hoboken, NJ: Wiley. https://doi.org/10.1002/9781118067475

Hutchison, L., Feder, M., Abar, B., \& Winsler, A. (2016). Relations between parenting stress, parenting style, and child executive functioning for children with ADHD or autism. Journal of Child and Family Studies, 25(12), 3644-3656. https://doi.org/10.1007/s10826$\underline{016-0518-2}$

Iacobucci, D., Schneider, M. J., Popovich, D. L., \& Bakamitsos, G. A. (2016). Mean centering helps alleviate "micro" but not "macro" multicollinearity. Behavior Research Methods, 48(4), 1308-1317. https://doi.org/10.3758/s13428-015-0624-X

Jones, H. A., Putt, G. E., Rabinovitch, A. E., Hubbard, R., \& Snipes, D. (2017). Parenting stress, readiness to change, and child externalizing behaviors in families of clinically referred children. Journal of Child and Family Studies, 26(1), 225-233. https://doi.org/10.1007/s10826-016-0547-X

Karver, M. S., Handelsman, J. B., Fields, S., \& Bickman, L. (2005). A Theoretical Model of Common Process Factors in Youth and Family Therapy. Mental Health Services Research, 7(1), 35-51. https://doi.org/10.1007/s11020-005-1964-4 
Karver, M. S., Handelsman, J. B., Fields, S., \& Bickman, L. (2006). Meta-analysis of therapeutic relationship variables in youth and family therapy: The evidence for different relationship variables in the child and adolescent treatment outcome literature. Clinical Psychology Review, 26(1), 50-65. https://doi.org/10.1016/j.cpr.2005.09.001

Kazdin, A. E., Mazurick, J. L., \& Siegel, T. C. (1994). Treatment outcome among children with externalizing disorder who terminate prematurely versus those who complete psychotherapy. Journal of the American Academy of Child \& Adolescent Psychiatry, 33(4), 549-557. https://doi.org/10.1097/00004583-199405000-00013

Kearney, M. W. (2017). Cross lagged panel analysis. The SAGE encyclopedia of communication research methods, 312-314.

King, G., Currie, M., \& Petersen, P. (2014). Child and parent engagement in the mental health intervention process: A motivational framework. Child and Adolescent Mental Health, 19(1), 2-8. https://doi.org/10.1111/camh.12015

Kirk, R. E. (2008). Statistics: An introduction. Belmont, CA: Thomas Higher Education.

Kutner, M. H., Nachtsheim, C. J., Neter, J., \& Li, W. (2005). Applied linear statistical models (5th ed.). New York: McGraw-Hill.

Laerd Statistics (2015a). Statistical tutorials and software guides. Retrieved from $\underline{\text { https://statistics.laerd.com/ }}$

Laerd Statistics (2015b). Moderator analysis with a dichotomous moderator variable using SPSS Statistics. Statistical tutorials and software guides. Retrieved from https://statistics.laerd.com/ 
Laerd Statistics (2018). Statistical tutorials and software guides. Retrieved from https://statistics.laerd.com/

Liles, B. D., Newman, E., LaGasse, L. L., Derauf, C., Shah, R., Smith, L. M., ... \& Lester, B. M. (2012). Perceived child behavior problems, parenting stress, and maternal depressive symptoms among prenatal methamphetamine users. Child Psychiatry and Human Development, 43(6), 943-957. https://doi.org/10.1007/s10578-012-0305-2

Littell, J. H., \& Girvin, H. (2005). Caregivers' readiness for change: Predictive validity in a child welfare sample. Child Abuse \& Neglect, 29(1), 59-80. https://doi.org/10.1016/j.chiabu.2004.08.004

Lovibond, S. H., \& Lovibond, P. F. (1995). Manual for the depression anxiety stress scales. Sydney: Psychology Foundation. https://doi.org/10.1037/t39835-000

Macaskill, N. D. (1989). Educating clients about rational-emotive therapy. In W. Dryden \& P. Trower (Eds.), Cognitive psychotherapy: Stasis \& change. (pp. 87-98). New York, NY: Springer Publishing Co.

Mansfield, E. R., \& Helms, B. P. (1982). Detecting multicollinearity. The American Statistician, 36(3a), 158-160. https://doi.org/10.1080/00031305.1982.10482818

Martinez, J. I., Lau, A. S., Chorpita, B. F., \& Weisz, J. R. (2017). Psychoeducation as a mediator of treatment approach on parent engagement in child psychotherapy for disruptive behavior. Journal of Clinical Child and Adolescent Psychology, 46(4), 573-587. https://doi.org/10.1080/15374416.2015.1038826 
Masi, M. V., Miller, R. B., \& Olson, M. M. (2003). Differences in dropout rates among individual, couple, and family therapy clients. Contemporary Family Therapy: An International Journal, 25(1), 63-75. https://doi.org/10.1023/A:1022558021512

May, C., Fletcher, R., Dempsey, I., \& Newman, L. (2015). Modeling relations among coparenting quality, autism-specific parenting self-efficacy, and parenting stress in mothers and fathers of children with ASD. Parenting: Science and Practice, 15(2), 119133. https://doi.org/10.1080/15295192.2015.1020145

McConnaughy, E. A., Prochaska, J. O., \& Velicer, W. F. (1983). Stages of change in psychotherapy: Measurement and sample profiles. Psychotherapy: Theory, Research \& Practice, 20(3), 368-375. https://doi.org/10.1037/h0090198

Melançon, F., Cossette, L., Smith, C., Beauvais-Dubois, C., Cyr, C., \& Smolla, N. (2019). Parenting stress of adoptive mothers, mother-child conflict, and behavior problems during adolescence among international adoptees. Journal of Family Psychology, 33(8), 988-993. https://doi.org/10.1037/fam0000542

Miller, A. L., Christenson, J. D., Glunz, A. P., \& Cobb, K. F. (2016). Readiness for change: Involving the family with adolescents in residential settings. Contemporary Family Therapy: An International Journal, 38(1), 86-96. https://doi.org/10.1007/s10591-016$\underline{9376-8}$

Miller, W. R., \& Rollnick, S. (2002). Motivational interviewing: Preparing people for change (Vol. 2). New York, NY: Guilford Press. https://doi.org/10.1097/01445442-200305000$\underline{00013}$ 
Miller, W. R., \& Rollnick, S. (2013). Motivational interviewing: Helping people change (3rd ed.). New York, NY: Guilford Press.

Miller, W. R., \& Rose, G. S. (2015). Motivational interviewing and decisional balance: Contrasting responses to client ambivalence. Behavioural and Cognitive Psychotherapy, 43(2), 129-141. https://doi.org/10.1017/S1352465813000878

Neece, C. L., Green, S. A., \& Baker, B. L. (2012). Parenting stress and child behavior problems: A transactional relationship across time. American Journal on Intellectual and Developmental Disabilities, 117(1), 48-66. https://doiorg.wvu.idm.oclc.org/10.1352/1944-7558-117.1.48

Niec, L. N., Barnett, M. L., Gering, C. L., Triemstra, K., \& Solomon, D. T. (2015). Differences in mothers' and fathers' readiness for change in parent training. Child \& Family Behavior Therapy, 37(3), 224-235. https://doi.org/10.1080/07317107.2015.1071980

Nock, M. K., \& Ferriter, C. (2005). Parent management of attendance and adherence in child and adolescent therapy: A conceptual and empirical review. Clinical Child and Family Psychology Review, 8(2), 149-166. https://doi.org/10.1007/s10567-005-4753-0

Nock, M. K., \& Kazdin, A. E. (2005). Randomized controlled trial of a brief intervention for increasing participation in parent management training. Journal of Consulting and Clinical Psychology, 73(5), 872-879. https://doi.org/10.1037/0022-006X.73.5.872

Norcross, J. C., Krebs, P. M., \& Prochaska, J. O. (2011). Stages of change. Journal of Clinical Psychology, 67(2), 143-154. https://doi.org/10.1002/jclp.20758 
Office for Victims of Crime (2017). Reaching Underserved Victims [Website]. Retrieved from https://www.ovc.gov/pubs/reporttonation2017/reaching-underserved-victim-groups.html

O'Hare, T. (1996a). Court-ordered versus voluntary clients: Problem differences and readiness for change. Social Work, 41(4), 417-422. https://doi.org/10.1093/sw/41.4.417

O'Hare, T. (1996b). Readiness for change: Variation by intensity and domain of client distress. Social Work Research, 20(1), 13-17. https://doi.org/10.1093/swr/20.1.13

Orne, M. T., \& Wender, P. H. (1968). Anticipatory socialization for psychotherapy: Method and rationale. The American Journal of Psychiatry, 124(9), 1202-1212. https://doi.org/10.1176/ajp.124.9.1202

PAR Inc. (n.d.). Parenting Stress Index ${ }^{\mathrm{TM}}$, Fourth Edition (PSI $\left.{ }^{\mathrm{TM}}-4\right)$ [Fact Sheet]. PAR Inc. http://www4.parinc.com/WebUploads/samplerpts/Fact\%20Sheet\%20PSI-4.pdf

Pearson Education (2016). Q-Global web-based administration, scoring, and reporting. SanAntonio, TX: PsychCorp.

Pearson. (n.d.). BASC-3 publication summary [PDF]. Retrieved from https://images.pearsonclinical.com/images/Assets/BASC-3/BASC3_Pub_Summary.pdf

Petrenko, C. L., Pandolfino, M. E., \& Roddenbery, R. (2016). The association between parental attributions of misbehavior and parenting practices in caregivers raising children with prenatal alcohol exposure: A mixed-methods study. Research in developmental disabilities, 59, 255-267. https://doi.org/10.1016/j.ridd.2016.09.005 
Petry, N. M. (2005). Stages of change in treatment-seeking pathological gamblers. Journal of Consulting and Clinical Psychology, 73(2), 312-322. https://doi.org/10.1037/0022$\underline{006 X .73 .2 .312}$

Phillips, B. A., Conners, F., \& Curtner-Smith, M. E. (2017). Parenting children with down syndrome: An analysis of parenting styles, parenting dimensions, and parental stress. Research in Developmental Disabilities, 68, 9-19. https://doi.org/10.1016/j.ridd.2017.06.010

Privitera, G. J. (2015). Statistics for the behavioral sciences, Second Edition. Sage.

Prochaska, J. O. (1979). Systems of psychotherapy: A transtheoretical analysis. Oxford: Dorsey. Prochaska, J. O., \& DiClemente, C. C. (1982). Transtheoretical therapy: Toward a more integrative model of change. Psychotherapy: Theory, Research \& Practice, 19(3), 276288. https://doi.org/10.1037/h0088437

Prochaska, J. O., \& DiClemente, C. C. (1983). Stages and processes of self-change of smoking: Toward an integrative model of change. Journal of Consulting and Clinical Psychology, 51(3), 390-395. https://doi.org/10.1037/0022-006X.51.3.390

Prochaska, J. O., \& DiClemente, C. C. (1992). Stages of change in the modification of problem behaviors. Progress in behavior modification, 28, 183-218.

Prochaska, J. O., DiClemente, C. C., \& Norcross, J. C. (1993). In search of how people change: Applications to addictive behaviors. Journal of Addictions Nursing, 5(1), 2-16. https://doi.org/10.3109/10884609309149692 
Prochaska, J. O., DiClemente, C. C., Velicer, W. F., Ginpil, S., \& Norcross, J. C. (1985).

Predicting change in smoking status for self-changers. Addictive Behaviors, 10(4), 395406. https://doi.org/10.1016/0306-4603(85)90036-X

Prochaska, J. O., Velicer, W. F., DiClemente, C. C., \& Fava, J. (1988). Measuring processes of change: Applications to the cessation of smoking. Journal of Consulting and Clinical Psychology, 56(4), 520-528. https://doi.org/10.1037/0022-006X.56.4.520

Prochaska, J. O., Velicer, W. F., Rossi, J. S., Goldstein, M. G., Marcus, B. H., Rakowski, W., ... \& Rossi, S. R. (1994). Stages of change and decisional balance for 12 problem behaviors. Health Psychology, 13(1), 39-46. https://doi.org/10.1037/0278-6133.13.1.39

Proctor, K. B., Brestan-Knight, E., Fan, J., \& Zlomke, K. R. (2017). Assessing parental readiness to change: A psychometric evaluation of the READI-SF in a community sample. Journal of Child and Family Studies, 27(4), 1054-1064. https://doi.org/10.1007/s10826-017-0952-9

Renner, L. M., Whitney, S. D., \& Easton, S. D. (2015). Profiles of self-reported parenting competence and stress among women with/without histories of childhood sexual abuse. Journal of Child and Family Studies, 24(3), 749-761. https://doi.org/10.1007/s10826-

\section{$\underline{013-9886-Z}$}

Reynolds, C., \& Kamphaus, R. (2015). Behavior assessment system for children, Third Edition. Bloomington, MN: Pearson Education. 
Rhee, K. E., De Lago, C. W., Arscott-Mills, T., \& Mehta, S. D. (2005). Factors associated with parental readiness to make changes for overweight children. Pediatrics, 116(1), e94e101. https://doi.org/10.1542/peds.2004-2479

Rodriguez, C. M. (2011). Association between independent reports of maternal parenting stress and children's internalizing symptomatology. Journal of Child and Family Studies, 20(5), 631-639. https://doi.org/10.1007/s10826-010-9438-8

Rutkowski, B. A., Gallon, S., Rawson, R. A., Freese, T. E., Bruehl, A., Crèvecoeur-MacPhail, D., ... Cotter, F. (2010). Improving client engagement and retention in treatment: The Los Angeles County experience. Journal of Substance Abuse Treatment, 39(1), 78-86. https://doi.org/10.1016/j.jsat.2010.03.015

Sameroff, A. (1975). Transactional models in early social relations. Human Development, 18(12), 65-79. https://doi.org/10.1159/000271476

Scott, K. L., \& Wolfe, D. A. (2003). Readiness to change as a predictor of outcome in batterer treatment. Journal of Consulting and Clinical Psychology, 71(5), 879-889. https://doi.org/10.1037/0022-006X.71.5.879

Sheras, P. L., Abidin, R. R., \& Konold, T. R. (1998). Stress index for parents of adolescents: Professional manual. Lutz, FL: Psychological Assessment Resources, Inc.

Sherman, M. D., \& Carothers, R. A. (2005). Applying the readiness to change model to implementation of family intervention for serious mental illness. Community Mental Health Journal, 41(2), 115-127. https://doi.org/10.1007/s10597-005-2647-8 
Shields, A. L., \& Hufford, M. R. (2005). Assessing motivation to change among problem drinkers with and without co-occurring major depression. Journal of Psychoactive Drugs, 37(4), 401-408. https://doi.org/10.1080/02791072.2005.10399813

Sirey, J. A. (2013). Engaging to improve engagement. Psychiatric Services, 64(3), 205. https://doi.org/10.1176/appi.ps.640301

Smith, J. P., \& Tran, G. Q. (2007). Does negative affect increase change readiness among college hazardous drinkers? Addictive Behaviors, 32(10), 2281-2285. https://doi.org/10.1016/j.addbeh.2007.01.019

Sowden, J. N., \& Olver, M. E. (2017). Sexual offender treatment readiness, responsivity, and change: Linkages to treatment completion and recidivism. Journal of Forensic Nursing, 13(3), 97-108. https://doi.org/10.1097/JFN.0000000000000160

Staudt, M. (2007). Treatment engagement with caregivers of at-risk children: Gaps in research and conceptualization. Journal of Child and Family Studies, 16(2), 183-196. https://doi.org/10.1007/s10826-006-9077-2

Sterrett, E., Jones, D. J., Zalot, A., \& Shook, S. (2010). A pilot study of a brief motivational intervention to enhance parental engagement: A brief report. Journal of Child and Family Studies, 19(6), 697-701. https://doi.org/10.1007/s10826-010-9356-9

Stone, L. L., Mares, S. H. W., Otten, R., Engels, R. C. M. E., \& Janssens, J. M. A. M. (2016). The co-development of parenting stress and childhood internalizing and externalizing problems. Journal of Psychopathology and Behavioral Assessment, 38(1), 76-86. https://doi.org/10.1007/s10862-015-9500-3 
Stone, L. L., Otten, R., Engels, R. C. M. E., Kuijpers, R. C. W. M., \& Janssens, J. M. A. M. (2015). Relations between internalizing and externalizing problems in early childhood. Child \& Youth Care Forum, 44(5), 635-653. https://doi.org/10.1007/s10566-014-9296-4

Tan, S., \& Rey, J. (2005). Depression in the young, parental depression and parenting stress. Australasian Psychiatry, 13(1), 76-79. https://doi.org/10.1111/j.1440-1665.2004.02155.x

Thomson, F. E., Bergman, P. B., \& Hay, M. (2018). Psychological Well-Being of Carers of Children with Type 1 Diabetes: A Comparison with Carers of Children without a Chronic Condition. Journal of Social Health and Diabetes, 6(02), 096-103. https://doi.org/10.1055/s-0038-1673741

Thulin, U., Svirsky, L., Serlachius, E., Andersson, G., \& Öst, L.-G. (2014). The effect of parent involvement in the treatment of anxiety disorders in children: A meta-analysis. Cognitive Behaviour Therapy, 43(3), 185-200. https://doi.org/10.1080/16506073.2014.923928

Timmer, S. G., Sedlar, G., \& Urquiza, A. J. (2004). Challenging children in kin versus nonkin foster care: Perceived costs and benefits to caregivers. Child Maltreatment, 9(3), 251262. https://doi.org/10.1177/1077559504266998

Torres, A., Frain, M., \& Tansey, T. N. (2019). The impact of motivational interviewing training on rehabilitation counselors: Assessing working alliance and client engagement A randomized controlled trial. Rehabilitation Psychology, 64(3), 328. https://doi.org/10.1037/rep0000267

Adept - Alcohol and Drug Education for Prevention and Treatment. (2017). Motivational Interviewing Tools and Techniques [PowerPoint slides]. University of Missouri Adept 
Program. https://adept.missouri.edu/wp-content/uploads/2017/06/Module-TwoMotivational-Interviewing-Tools-and-Techniques.pdf

Wade, M., \& Andrade, B. F. (2015). Validation of a measure of parental readiness for treatment in a clinical sample of children with disruptive behavior. Journal of Psychopathology and Behavioral Assessment, 37(2), 184-195. https://doi.org/10.1007/s10862-014-9458-6

Walitzer, K. S., Dermen, K. H., \& Connors, G. J. (1999). Strategies for preparing clients for treatment: A review. Behavior Modification, 23(1), 129-151.

\section{https://doi.org/10.1177/0145445599231006}

Webb, H. J., Thomas, R., McGregor, L., Avdagic, E., \& Zimmer-Gembeck, M. J. (2017). An evaluation of parent-child interaction therapy with and without motivational enhancement to reduce attrition. Journal of Clinical Child and Adolescent Psychology, $46(4), 537-550$.

https://doi.org/10.1080/15374416.2016.1247357

Weisberg, S. (2014). Applied linear regression (4th ed.). New York: Wiley.

West, S. G., Finch, J. F., \& Curran, P. J. (1995). Structural equation models with nonnormal variables: Problems and remedies. In R. H. Hoyle (Ed.), Structural equation modeling: Concepts, issues and applications (pp. 56-75). Newbury Park, CA: Sage.

Wherry, J. N., Huey, C. C., \& Medford, E. A. (2015). A national survey of child advocacy center directors regarding knowledge of assessment, treatment referral, and training needs in physical and sexual abuse. Journal of Child Sexual Abuse: Research, Treatment, 
\& Program Innovations for Victims, Survivors, \& Offenders, 24(3), 280-299. https://doi.org/10.1080/10538712.2015.1009606

Wiener, J., Biondic, D., Grimbos, T., \& Herbert, M. (2016). Parenting stress of parents of adolescents with attention-deficit hyperactivity disorder. Journal of Abnormal Child Psychology, 44(3), 561-574. https://doi.org/10.1007/s10802-015-0050-7

Williford, A. P., Calkins, S. D., \& Keane, S. P. (2007). Predicting change in parenting stress across early childhood: Child and maternal factors. Journal of Abnormal Child Psychology, 35(2), 251-263. https://doi.org/10.1007/s10802-006-9082-3

Willoughby, F. W., \& Edens, J. F. (1996). Construct validity and predictive utility of the stages of change scale for alcoholics. Journal of Substance Abuse, 8(3), 275-291. https://doi.org/10.1016/S0899-3289(96)90152-2

Wolfteich, P., \& Loggins, B. (2007). Evaluation of the Children's Advocacy Center model: Efficiency, legal and revictimization outcomes. Child \& Adolescent Social Work Journal, 24(4), 333-352. https://doi.org/10.1007/s10560-007-0087-8

Woodward, L. J., \& Fergusson, D. M. (2001). Life course outcomes of young people with anxiety disorders in adolescence. Journal of the American Academy of Child \& Adolescent Psychiatry, 40(9), 1086-1093. https://doi.org/10.1097/00004583-200109000$\underline{00018}$

Zemore, S. E. (2012). The effect of social desirability on reported motivation, substance use severity, and treatment attendance. Journal of Substance Abuse Treatment, 42(4), 40012. https://doi.org/10.1016/j.jsat.2011.09.013 


\section{APPENDICES}

Appendix A: IRB Approval

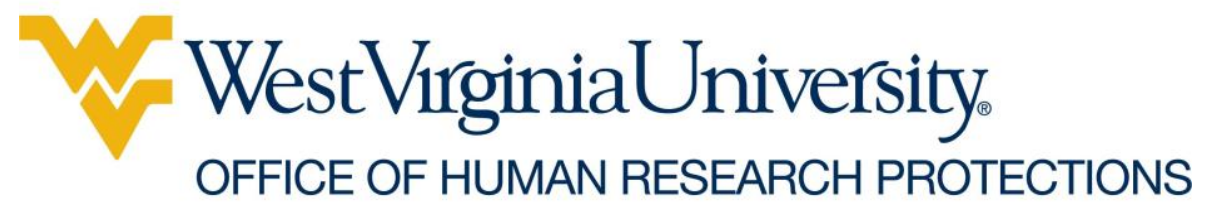

886 Chestnut Ridge Road Morgantown, WV 26506

Flex Model Approval

$02 / 25 / 2021$

To: Monica Leppma

From: WVU Human Research Protection Program

Protocol Type: NHSR / Flex

Approval Date: 02/25/2021

Submission Type: Initial

Expiration Date: 02/24/2026

Funding: N/A

WVU Protocol \#: 2009108587

Protocol Title: Parenting Stress as A Moderator of the Relationship Between Child Emotional and Behavioral Problems and Parental Readiness for Change

The West Virginia University Institutional Review Board has determined that your submission of protocol 2009108587 qualifies for approval under the WVU Flexibility Review Model.

- FLEX: The review of the above referenced protocol was conducted in accordance with the ethical principles of the Belmont Report. The protocol meets the criteria for approval Flex Model Review because the project does not have federal funding, is considered to be minimal risk, and falls within the guidelines of the WVU Flex Model as of this approval date. 
The following documents were reviewed and approved for use as part of this submission. Only the documents listed below may be used in the research. Please access and print the files in the Notes \& Attachments section of your approved protocol.

- PRFCS-SF_final.docx

- PRS-A Age 12-21yrs old.pdf

- PRS-C Child Ages 6-11.pdf

- PRS-P Preschool Ages 2-5.pdf

- PSI-4 Birth to 12.pdf

- SIPA Item Booklet.pdf

\section{WVU IRB approval of protocol 2009108587 will expire on 02/24/2026.}

1. There is no continuing review option for Flex protocols. If this protocol is still active after five (5) years and you intend to continue, you will need to resubmit a new protocol for approval no less than three (3) weeks prior to the expiration date in order to ensure uninterrupted study continuation.

2. Amendments are not accepted on Flex protocols and are not required as long as there is no increase to risk to participants and changes do not exclude the study from Flex inclusion criteria.

3. The IRB must be notified if there is an increase in risk to participants or if federal funding is aw arded to conduct research under this protocol. In any of these cases, this protocol must be submitted as a new submission in WVU+kc.

4. Unanticipated, serious adverse events and/or side effect(s) encountered at WVU or an affiliate site that are related to the research must be reported to the WVU IRB within five (5) days using the Notify IRB action in WVU+kc.

5. Any Unanticipated Problem or UPIRTSO or other research related event resulting in new or increased risk of harm to study subjects, occurring at WVU or an affiliate site, must be reported to the WVU IRB within five (5) days using the Notify IRB action in WVU+kc.

6. All research personnel performing tasks related to the research must complete and remain current for the required training as applicable to the protocol referenced above.

7. Only Flex Consent Forms with the WVU+kc watermark may be used to consent participants.

The WVU Office of Human Research Protections will be glad to provide assistance to you throughout the research process. Please feel free to contact us by phone at 304.293.7073 or by email at IRB@mail.wvu.edu.

Sincerely, 
PARENTING STRESS AS A MODERATOR

105

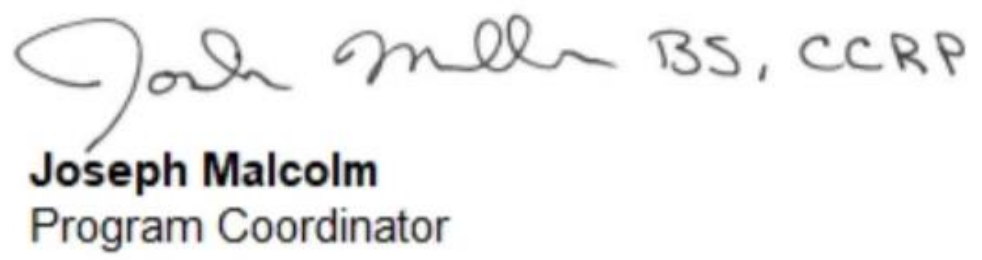




\section{Appendix B: PRFCS-SF}

ID\#

IN PRE POST FUP

Date:

Your relationship to the child here for treatment (e.g., mother, stepfather):

Each statement describes how a person might feel when thinking about their parenting. Please indicate the extent to which you tend to agree or disagree with each statement. In each case, make your choice in terms of how you feel right now, not what you have felt in the past or would like to feel.

Strongly
Disagree Disagree Undecided $\underline{\text { Agree }} \underline{\text { Agree }}$

1. As far as I'm concerned, I don't need to change how I take care of my child...

2. I think I might be ready to improve how I take care of my child......

$\begin{array}{lllll}1 & 2 & 3 & 4 & 5\end{array}$

3. I am trying to learn how to take care of my child

$2 \quad 3 \quad 4 \quad 5$

4. I take perfect care of my child. It doesn't make sense for me to change..

5. I've been thinking that I might want to learn the best ways to help my child. .

6. At times it's hard to be a good parent, but l'm working on it

$2 \quad 3 \quad 4 \quad 5$

7. Being here is pretty much a waste of time for me because I already know how to take care of my child

$2 \quad 3 \quad 4 \quad 5$

8. I'm hoping to learn to better understand my interactions with my child.

$\begin{array}{lllll}1 & 2 & 3 & 4 & 5\end{array}$

9. I guess my parenting isn't perfect, but

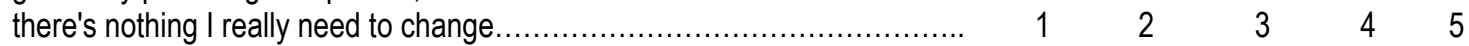

10. I am really working hard to change how I take care of my child

$\begin{array}{lllll}1 & 2 & 3 & 4 & 5 \\ 1 & 2 & 3 & 4 & 5 \\ 1 & 2 & 3 & 4 & 5 \\ 1 & 2 & 3 & 4 & 5 \\ 1 & 2 & 3 & 4 & 5 \\ 1 & 2 & 3 & 4 & 5 \\ 1 & 2 & 3 & 4 & 5 \\ 1 & 2 & 3 & 4 & 5\end{array}$

11. My parenting needs improvement and I really think I should work on it

$\begin{array}{lllll}1 & 2 & 3 & 4 & 5 \\ 1 & 2 & 3 & 4 & 5 \\ 1 & 2 & 3 & 4 & 5 \\ 1 & 2 & 3 & 4 & 5 \\ 1 & 2 & 3 & 4 & 5 \\ 1 & 2 & 3 & 4 & 5 \\ 1 & 2 & 3 & 4 & 5 \\ 1 & 2 & 3 & 4 & 5\end{array}$

12. Even though I am not always successful in changing how I interact with my child, I am at least working on it

13. I wish I had more ideas on how to take care of my child better....

14. Maybe parent classes will help me handle my child better

15. I may need to change how I am as a parent, but I don't think so

$2 \quad 3 \quad 4 \quad 5$

16. Anyone can talk about changing how they are as a parent; I'm actually doing something about it....

17. I am actively trying to change how I care for my child. 


\title{
Appendix C: Demographics Form
}

\author{
Client Name: \\ Case Number: \\ Supplement - Child/ Adolescent Therapy Questionnaire
}

Prenatal/ Birth Information:

List any complications that occurred when the mother was pregnant with this child (e.g., excessive vomiting, threatened miscarriage, infections, toxemia, illnesses,

hospitalization):

a) Did the mother smoke/ use tobacco during the pregnancy?

b) Did the mother drink alcohol during the pregnancy?

c) Did the mother use drugs/medications during the pregnancy?

If you answered yes to any of the questions a-c, please describe what was consumed and how much was used.

Was the pregnancy a full term pregnancy? If the child was born premature or late please explain.

Regarding the delivery, please check all that apply:

_ Vertex (normal) __ Breech _ Caesarean _ Induced

Were there any complications during the delivery? __ If you answered yes, please explain.

How much did your child weigh at birth?

What was the child's Apgar score?

Were there any birth defects?

How many days did the baby stay in the hospital after the delivery?

Infancv/ Toddler Period:

What was your child like during the first two years of his or her life? Please check all that apply.

Liked to be held

Liked to cuddle

Did not Like to be touched

Aggressive

Excessive restlessness

Overly active

Colic

Easy to Bond with

Other (please specify) 


\section{Developmental Information:}

Regarding your child's language development (e.g., saying his/her first words), was it early, on time, or late?

Regarding your shild's motor development (e.g., sitting without support, crawling, walking), was it early, on time, or late?

Were there any skills that your child was exceptionally slow at learning?

Is your child blaidder and bowel trained both during the day and at night?

\section{Home Behavior (Current):}

All children/ adolescents exhibit to some degree the kinds of behaviors listed below. Check those that you believe your child exhibits to an excessive or exaggerated degree when compared to other children his or her age.

hyperactivity (high activity level)

poor atterition span

impulsivity (poor self control)

doesn't think before she or he acts

temper outburst

low frustration tolerance (gets upset easily)

doesn't listen when spoken to

problems following directions

sassy/tallis back to parents

gets upset if doesn't get his or her way

change in eating habits

threatens 1o harm/ kill himself or others aggressive

poor memory

heedless to danger

steals

lies

cries easily

worries excessively

has a lot of fears

excessive number of accidents

problems with sleeping

makes negative self statements

obsessions/ compulsions

Describe your child's mood. (e.g., happy, sad, angry) 
How does your child get along with family members?

What are your child's main hobbies and interests?

How do you dis:ipline your child?

\section{School Behavior (Current):}

Does your child like school? Why or why not?

How would you rate your child's overall level of intelligence compared to other children? Below average Average Above Average

Has your child ever had to repeat a grade? If so, when

Is your child cunently in a special education class?

Does your child inave an IEP? Does your child see the school counselor?

Is your child rectiving any special therapy or remedial work in school?

Is your child struggling/ doing poorly in any subjects in school?

What are your child's grades?

Briefly describe any problems that your child is having at school.

\section{Peer Relationships (Current):}

Does your child have close friends? How many?

How often does your child play with friends outside of school?

Does your child €et along with his peers? (Please describe any problems.)

Does your child telong to any teams or groups? 


\section{Medical History:}

If your child's medical history includes any of the following, please note the age when the incident or illness occurred and any other pertinent information.

Childhood disea:ses

Operations

Hospitalizations

Head Injuries (?) Pease explain whether your child was unconscious

\section{Seizures}

Eye problems

Ear problems

Poisoning

Has your child e'ver seen another psychologist, counselor, or psychiatrist?

$\overline{\text { Has your child } \mathrm{e}}$ /er been hospitalized for any psychiatric problems (e.g., suicide attempt, extreme aggressive behavior)?

\section{Current Medical Status:}

Present height Present weight

Present Illness(esi) for which your child is being treated

Medications that your child is taking on an ongoing basis

Family History -... Mother:

Has the mother had any

Learning problems

Behavior problems

Emotional problems

Medical frroblems

Family History -- Father:

Has the father had any

Learning problems

Behavior problems

Emotional problems

Medical froblems 


\begin{tabular}{|c|c|}
\hline & $\begin{array}{c}\text { Monongalia County Child Advocacy Center } \\
909 \text { Greenbag Road } \\
\text { Case Number: }\end{array}$ \\
\hline
\end{tabular}

General Child/ Adolescent Information Form

Today's Date:

Child Name:

Address: City/ State/ Zip Code:

Date of Birth:

Age:

Child's Primary Language:

Telephone \#:

Is it $\mathrm{OK}$ to contact you at this number and leave a message?

Mother's Name: Mother's Age: Occupation:

Father's Name: Father's Age: Occupation:

Legal Guardian(s):

Who referred you here today?

Why did you come to the Monongalia County Child Advocacy Center today? What services would you like? 
Demographic Information:

Race or Ethnicity: (Please check)

$\begin{array}{cccc}\text { African-American } & \text { Asian } \\ \text { Hispanic/ Latino } & - & \text { Bi-/Racial } \\ - & \text { White } & - & \text { Other }\end{array}$

School:

Grade:

Daycare Center/ Provider:

Does your child have any learning problems, disabilities, or delays? If yes, please explain.

Annual Household Income:

Less than $\$ 10,000-\$ 10,000-\$ 19,999-\$ 20,000-\$ 29,999$
$-\$ 30,000-39,999-\$ 40,000-\$ 49,000-\$ 50,000-\$ 59,999$
$\$ 60,000$ or Greater

Insurance Type:

Social Security Number:

Who does your child live with?

\begin{tabular}{|l|l|l|}
\hline Individual's Name & $\begin{array}{l}\text { Relationship to Your } \\
\text { Child }\end{array}$ & Age \\
\hline & & \\
\hline & & \\
\hline & & \\
\hline & & \\
\hline
\end{tabular}

Does your family have an open Family Court Case?

Does your family have an open Child Protective Services Case?

Does your child take any medication? If yes, please list. If yes, please list the therapist. 
Does your child have any emotional or behavioral problems?

If yes, please explain.

Please list any other family members (e.g., siblings) or significant people in the child's life?

Who is completing this form? 


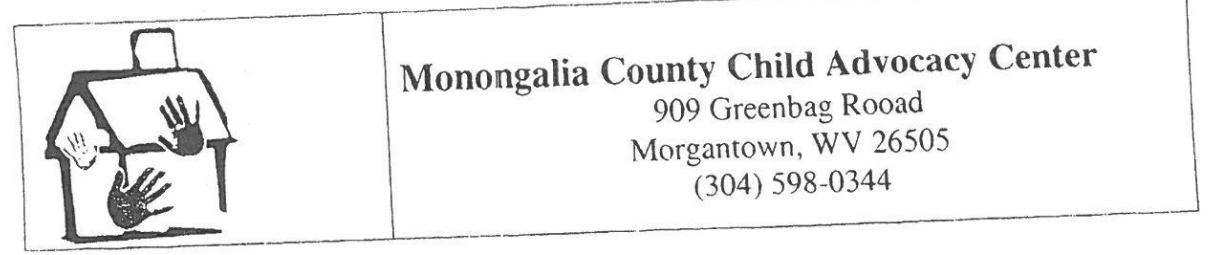

Case Number:

\section{General Adult Information Form}

Today's Date

Name Gender: Male or _Female

Address: City/ State/ Zip Code:

Date of Birth: Telephone \#:

Is it OK to contact you at this number and leave a message?

Who referred you here today?

Why did you come to the Monongalia County Child Advocacy Center today? What services would you like?

Would you like any information about the following? Please check the appropriate category.

housing assistance with transportation assistance with paying bills

\section{Demograhpic Information:}

Race or Ethnicity: (Please check)

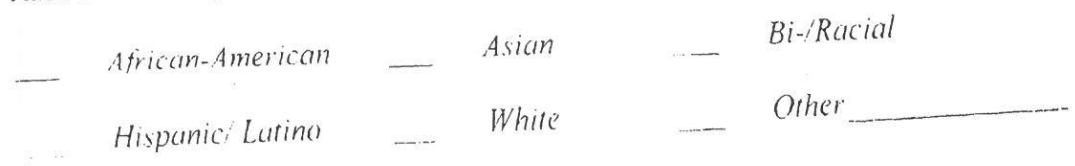


Present Marital Status: (Please check all that apply)

Single
Divorced
Other

Years of formal education completed: (Please check the highest level of schooling you achieved):

Grade School Middle School High School
Trade School Undergraduate College Education
Master's Program in College Doctoral Program in College
Other

Annual Household Income:

Lessthan $\$ 10.000-\$ 10,000-\$ 19.999-\$ 20,000-\$ 29,999$
$-\$ 30.000-39,999-\$ 40,000-\$ 49,000+\$ 50.000-\$ 59,999$
$\$ 60.000$ or Greater

Occupation:

Social Security Number:

Who do you live with?

\begin{tabular}{|l|l|l|}
\hline Individual's Name & Relationship to You & Age \\
\hline & & \\
\hline & & \\
\hline & & \\
\hline
\end{tabular}

Do you have any children? If yes. please list their names and ages.

Do you have an open Family Court Case?

Do you have an open Child Protective Services Case? 
Do you take any medication? If yes. please list

Do you currently see a therapist? If yes, please list the therapist

Do you have any emotional or behavioral problems? If yes, please explain. 


\section{Appendix D: Child and Guardian Demographics}

\section{Table 1}

Child and Guardian Demographics

$(S D)$

Child Demographics

Age

9.0

Sex

Female

Male

$38(59.4)$

$26(40.6)$

Race*

African American

$3(4.7)$

Bi-Racial

$3(4.7)$

Hispanic/Latino

$1(1.6)$

White

$56(87.5)$

Guardian Demographics

Age

41.58

Sex

Female

(10.36)

Male

$48(75.0)$

$16(25.0)$

Race

African American

$1(1.6)$

Bi-Racial

$3(4.7)$

Hispanic/Latino

$1(1.6)$

White

$59(92.2)$

Guardian's Relationship to Child

Biological Father

$10(15.6)$

Biological Mother

$27(42.2)$

Foster Father

$2(3.1)$

Foster Mother

$4(6.3)$

Grandfather

$1(1.6)$

Grandmother

$11(17.2)$

Uncle

$2(3.1)$

Aunt

$3(4.7)$

Other

$4(6.3)$

Guardian's Marital Status*

Single

17 (26.6) 


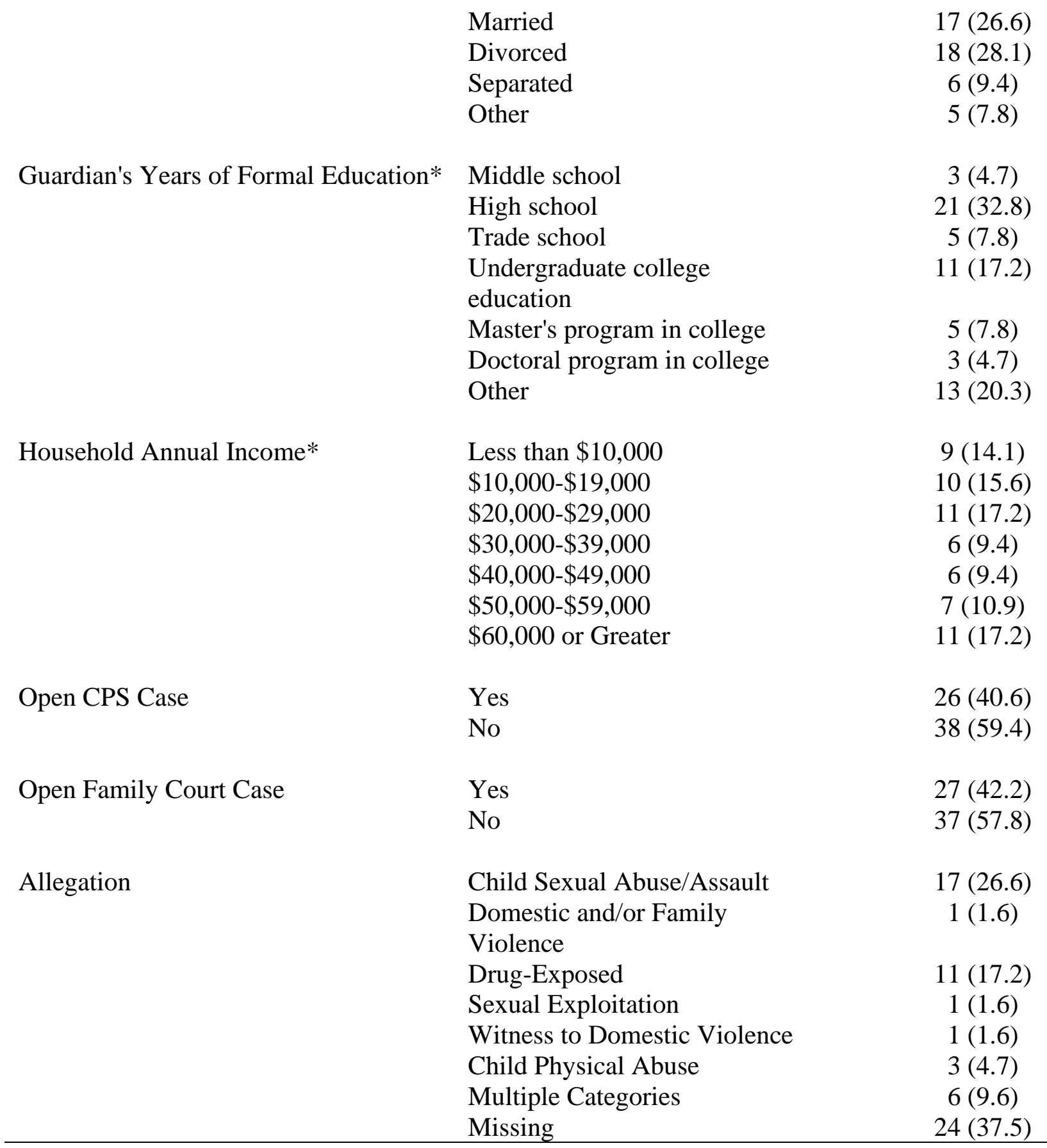

Note. $* N$ varies due to missing values. 
Appendix E: Means, standard deviations, and bivariate correlations among Parental

Readiness for Change, Parenting stress, Internalizing Problems, and Externalizing

\section{Problems}

\section{Table 2}

Means, standard deviations, and bivariate correlations among Parental Readiness for Change, Parenting stress, Internalizing Problems, and Externalizing Problems

\begin{tabular}{lllll}
\hline Variable & 1. & 2. & 3. & 4. \\
\hline M & 29.01 & 52.83 & 55.97 & 57.23 \\
SD & 11.55 & 24.75 & 13.03 & 12.85 \\
1. Readiness for Change & -- & & & \\
2. Parenting Stress & $.422^{* *}$ & -- & & \\
3. Internalizing Problems & $.295^{*}$ & $.508^{* *}$ & -- & \\
4. Externalizing Problems & $.356^{* *}$ & $.636^{* *}$ & $.599 * *$ & --
\end{tabular}

Note. Spearman's Rho was used for Externalizing Problems correlations and Pearson's r was used for Readiness, Parenting Stress and Internalizing Problems. $*<.05 . * *<.001$ 
Appendix F: Multiple Regression Predicting Parenting Stress From Child Age, Family

Court Case Status, Internalizing problems, Externalizing problems, and Parenting Stress

Table 3

Multiple Regression Predicting Parenting Stress From Child Age, Family Court Case Status, Internalizing problems, Externalizing problems, and Parenting Stress

\begin{tabular}{|c|c|c|c|c|}
\hline \multirow[b]{3}{*}{ Variable } & \multicolumn{4}{|c|}{ Parental Readiness for Change } \\
\hline & \multicolumn{2}{|c|}{ Model 1} & \multicolumn{2}{|c|}{ Model 2} \\
\hline & $\beta$ & $t$ & $\beta$ & $t$ \\
\hline Constant & & $10.15 * *$ & & $6.90 * *$ \\
\hline Child Age & -.39 & $-3.32 *$ & -.37 & $-3.15^{*}$ \\
\hline Open Family Court Case & .17 & 1.44 & .21 & 1.88 \\
\hline Internalizing Problems & & & .21 & 1.45 \\
\hline Externalizing Problems & & & .08 & .48 \\
\hline Parenting Stress & & & .13 & .90 \\
\hline
\end{tabular}

Note. $N=64 . * p<.05, * * p<.001$. 
Appendix G: Hierarchical Multiple Regression Predicting Parenting Stress From

Internalizing and Externalizing problems

Table 4

Hierarchical Multiple Regression Predicting Parenting Stress From Internalizing and Externalizing problems

\begin{tabular}{llclc}
\hline & \multicolumn{4}{c}{ Parenting Stress } \\
\cline { 2 - 5 } Variable & \multicolumn{3}{c}{ Model 1 } & Model 2 \\
\cline { 2 - 5 } Constant & $\mathrm{B}$ & $\mathrm{B}$ & $\beta$ \\
Internalizing Problems & -7.48 & & -32.86 & .22 \\
Externalizing Problems & & .56 & .474 & .54 \\
$R^{2}$ & & 1.05 & \\
$F$ & .313 & .503 & \\
$\Delta R^{2}$ & $27.83^{* *}$ & & $22.87 * *$ \\
$\Delta F$ & .313 & .190 & \\
\hline Note. $N=64 . * p<.05, * * p<.001$. & & $22.87 * *$ \\
\end{tabular}




\section{CURRICULUM VITAE}

Connor Fais, M.A.

Phone: (614) 561-4354

Email: connor.fais@gmail.com

2079 Solomon Sq., Delaware, OH 43015

\section{$\underline{\text { Education }}$}

West Virginia University, Morgantown, WV (08/2015-Present)

Counseling Psychology, PhD

University of Dayton, Dayton, $\mathrm{OH}$ (Graduated: 12/2014)

Clinical Psychology, MA

Ohio University, Athens, OH (Graduated: 06/2012)

Psychology, BA

\section{Clinical Experience}

Doctoral Intern, Astor Services for Children and Families, Rhinebeck/Poughkeepsie, NY (08/2020-08/2021) 50-55 Hrs./Week

- Lead weekly DBT-informed outpatient social skills group for young adults via telehealth/Zoom

- Co-lead weekly DBT-informed inpatient group therapy for adolescents

- Conduct psychological assessment with outpatient and inpatient clients

- Worked as a child, adolescent and family outpatient clinician 2 days per week (33-35 client caseload)

- Worked as an adolescent inpatient clinician 3 days per week (5 client caseload)

- Lead monthly multidisciplinary treatment team meetings for inpatient clients

- Responded to and perform crisis intervention with inpatient clients on an as-needed basis

- Participated in program evaluation, didactics, monthly trainings, and outpatient team meetings

- Supervisors: Jennifer Brody, PsyD and Meghan Brown, PsyD 
Doctoral Trainee, Cardinal Pediatrics, Morgantown, WV (06/2019-08/2020) 2022 Hrs./Week

- Administered psychological assessments for youth (e.g., intelligence testing, achievement testing, ADHD testing, phonological processing, and symptom inventories)

- Wrote integrated psychological reports for youth testing clients

- Provided individual, dyadic, and parent counseling services to youth and families

- Conducted psychological testing feedback sessions to discuss psychological testing, results, and discuss recommendations

- Supervisor: Traci Berry-Harris, PhD

Assessment Graduate Assistant and Advanced Clinical Trainee, West Virginia University Carruth Center for Psychological and Psychiatric Services, Morgantown, WV (08/2019-Present) 20 Hrs./Week

- Administered psychological assessments for college students (i.e., tests of intelligence, achievement, ADHD, and executive functioning)

- Wrote integrated psychological reports

- Performed weekly triage and risk assessments

- Supervisor: Narayan Gold, PhD

Advanced Clinical Trainee, West Virginia University Carruth Center for

Psychological and Psychiatric Services, Morgantown, WV (08/2018-05/2019) 16-20 Hrs./Week

- Provided individual counseling services to West Virginia University students under the clinical supervision of licensed psychologists

- Performed associated clinical duties including triage and risk assessments, drop-in hours, participated in weekly group and in individual supervision, completed intake assessments, and clinical documentation

- Supervisors: Narayan Gold, PhD, Shane Chaplin, PhD, and Christine Simpson, PsyD

Clinical Trainee/Therapist, Monongalia County Child Advocacy Center, Morgantown, WV (07/2016-Present) 16-20 Hrs./Week

- Provided individual, dyadic, and parenting therapy services under the clinical supervision of Laura Capage, $\mathrm{PhD}$, licensed psychologist, and Executive Director of the Monongalia County Child Advocacy Center

- Performed associated duties including clinical evaluations, treatment planning, and case conceptualization 
- Collaborated with supervisor to provide written courtroom recommendations for child custody cases

- Consulted and collaborated with parents, teachers, therapists, family advocates, and school administrators as part of a multidisciplinary service team

- Supervisor: Laura Capage, $\mathrm{PhD}$

Residential Specialist/Qualified Mental Health Specialist, Daybreak (emergency youth shelter and transitional housing center), Dayton, OH (09/201408/2015) 40 Hrs./Week

- Supervised clients in the youth shelter

- Taught daily living skills and promoted developmental assets

- Processed intake and exit paperwork with clients at the shelter

- Wrote progress notes tracking client behavior and functioning

Masters Clinical Trainee, Daybreak (emergency youth shelter and transitional housing), Dayton, OH (09/2012-07/2014), 16-20 Hrs./Week, approximately 1,300 onsite hours

- Provided case management for youth in a community housing program

- Trained in trauma-informed care

- Taught clients daily living skills and developmental assets

- Managed a behavioral contingency program for residential clients

\section{$\underline{\text { Assessment Experience }}$}

I have written approximately 20 integrative assessment reports and have conducted assessment in a college counseling and community mental health settings. Below is a list of assessments that $\mathrm{I}$ have had practical experience administering with past clients:

Intelligence \& Achievement Tests

- WISC-V Wechsler Intelligence Scale for Children

- Wechsler Individual Achievement Test (WIAT-III)

- WAIS-IV Wechsler Adult Intelligence Scale 4th Edition

- Woodcock-Johnson IV Test of Achievement

Measures of Executive Functioning

- Conners' Continuous Performance Test 3rd Edition

- D-KEFS Delis-Kaplan Executive Function System 
- Nonverbal Medical Symptom Validity Test

- IVA-2 - Evaluate Visual and Auditory Attention

Projective Tests

- Child and Adolescent Sentence Completion Tests

- Roberts Apperception Test for Children: 2

- House-Tree-Person

Phonological Processing

- Comprehensive Test of Phonological Processing -2 (CTOPP-2)

\section{Child/Adolescent Symptom Measures}

- Conners 3 Assessment

- BASC-3 Behavior Assessment System for Children 3rd Ed

- BDI-II Beck Depression Inventory-II

- Beck Anxiety Inventory (BAI)

- Child PTSD Symptom Scale for DSM-5

- ASRS Autism Spectrum Rating Scales

- Children's Depression Inventory 2

- PHQ-9 Depression Test Questionnaire

- Pittsburgh Sleep Quality Index

- Screen for Child Anxiety Related Disorders (SCARED)

- Personality Inventory for Youth

\section{$\underline{\text { Research Publications }}$}

Mamboleo, G., Dong, S., \& Fais, C. (2020). Factors associated with disability self-disclosure to their professors among college students with disabilities. Career Development and Transition for Exceptional Individuals, 43(2), 78-88. doi:10.1177/2165143419893360

Blake, J., Yaghmaian, R., Brooks, J., Fais, C., \& Chan, F. (2018). Attachment, hope, and participation: Testing an expanded model of Snyder's hope theory for prediction of participation for individuals with spinal cord injury. Rehabilitation Psychology, 63(2), 230-239.

doi:10.1037/rep0000204 
Fais, C., Lutz-Zois, C., \& Goodnight, J. (2017). Mediators and moderators of the association between stalking victimization and psychological distress. Journal of Interpersonal Violence, Advanced online publication. doi: $10.1177 / 0886260517699951$

\section{$\underline{\text { Research, Posters, \& Symposiums }}$}

Swiger, T., Molder, A., \& Fais, C. (2018, April). The Opioid Epidemic:

Counseling Psychology's Role in Prevention and Recovery. Roundtable moderated at the 2018 Great Lakes Regional Counseling Psychology Research Conference in Kalamazoo, MI.

Latorre, C., Molder, A., \& Fais, C. (2018, April). Familism and Self-Stigma as Barriers to Seeking Psychological Care. Poster presented at the 2018 Great Lakes Regional Counseling Psychology Research Conference in Kalamazoo, MI.

Fais, C., \& Molder, A. (2017, April). Introduction and Review of Racial Trauma Research. In C. Fais (Chair), Recognizing racial trauma: Research, clinical implications, and advocacy work. Symposium presented at the 2017 Great Lakes Regional Counseling Psychology Conference in Muncie, IN.

Fais, C., \& Swiger, T. (2016, April). Can I really forgive and forget? In S. B. Milam (Chair), Forgiveness and hope as pathways to decrease prejudice and increase inclusivity in a multicultural society following terrorism. Symposium presented at the 2016 Great Lakes Regional Counseling Psychology Conference in Bloomington, IN.

Fais, C. \& Zois, C. (2015, August). Mediators and Moderators of The Association Between Stalking Victimization and Psychological Distress. Poster presented at The American Psychological Association Annual Convention in Toronto, ON. 


\section{$\underline{\text { Research Experience }}$}

\section{Doctoral Dissertation}

West Virginia University, WV (08/2018-Present)

"Parenting Stress as A Moderator of the Relationship Between Child Emotional and Behavioral Problems and Parental Readiness for Change"

- Dissertation successfully proposed 05/2019

\section{Doctoral Research}

- Contributed to research projects with Drs. George Mamboleo, PhD, John Blake, $\mathrm{PhD}$, and Lisa Platt, $\mathrm{PhD}$

Master's Thesis, University of Dayton, OH (09/2012-01/2016)

Mediators and Moderators of The Association Between Stalking Victimization and Psychological Distress

- Published 3/28/2017 in the Journal of Interpersonal Violence

Research Assistant, Center for Evaluation and Treatment of Anxiety, Athens, OH (04/2010-04/2012) 4-6 Hrs./Week

- Entered participant data

- Practiced ethical safeguards for maintaining confidentiality

- Assisted Clinical Psychology Ph.D. students with experiment tasks

Research Assistant, Structure, Concepts and Perception Laboratory, Athens, $\mathrm{OH}$ (11/2009-04/2010), 2-4 Hrs./Week

- Briefed, ran, and debriefed psychology research participants

\section{Teaching Experience}

Graduate Teaching Assistant, West Virginia University Department of Child Development and Family Studies, Morgantown, WV (08/2018-05/2019)

20 Hrs./Week

- Served as teaching assistant for an undergraduate-level research methods course in the department of Child Development and Family Studies

- Provided feedback and support, and served as a substitute instructor 
- Performed other teaching duties such as grading, course development and student communication

Graduate Teaching Assistant, Instructor, West Virginia University Department of Psychology, Morgantown, WV (08/2017-Present) 20 Hrs./Week

- Prepared and taught two sections of Abnormal Psychology (PSYC 281, approx. 50 student class size)

- Performed other teaching duties such as exam preparation, grading, student communication, and received weekly teaching supervision meetings

- Engaged in teaching career development tasks including development of a teaching philosophy

- Supervised two undergraduate teaching assistants on tasks such as grading and guest lecture

Graduate Teaching Assistant, Instructor, West Virginia University Department of Counseling, Rehabilitation, Counseling, and Counseling Psychology, Morgantown, WV (08/2015-08/2017) 20 Hrs./Week

- Served as instructor of two online courses: Career and Lifespan Development (COUN 405) and Introduction to Counseling (COUN 303)

- Provided feedback, support, and instruction to students

- Engaged in online course development with faculty supervisor

\section{Volunteer Experiences}

Community Volunteer/Big Brother, Big Brothers Big Sisters, Dayton, OH (10/2012-08/2015) 4-10 Hrs./Month

- Coordinated and participated in monthly activities for healthy youth development with "little brother"

Student Volunteer, Dublin Counseling Center, Dublin, OH (11/2010-12/2010 \& 06/2011-08/2011) 10-12 Hrs./Week

- Shadowed a counselor during an alcohol intervention program

- Preformed office duties including filing, copy making and billing tasks

- Observed group supervision and staff meetings 


\section{Selected Trainings}

Attachment Theory and Clinical Interventions - Online Training - 1 Hour Presented by Raven Maldonado-Brown, LMHC on 02/16/2021.

SP-TIE: Introduction to Cognitive Therapy for Suicide Prevention- Online Training - 1 Hours 22 Minutes - 1/25/2021

Comprehensive Suicide Risk Assessment by Center for Practice Innovations - Online Training - 30 Minutes - 11/22/2020

Engaging Families and Social Supports in Working with Suicidal Individuals by Center for Practice Innovations - Online Training - 1 Hour - 10/26/2020

Motivational Interviewing: Interactive Practice Scenarios

by Center for Practice Innovations - Online Training - 9/4/2020

Screening, Intervention and Referral to Treatment (SBIRT) - Online Training - 9/4/2020

Trauma-Focused Cognitive Behavioral Therapy - Online Training - 11 Hours $-7 / 13 / 2020$

PCIT For Traumatized Children Web Course Presented by University of California Davis - Online Training - 10 Hours - 7/6/2020

Eberly College of Arts \& Sciences School of Social Work's Play therapy + trauma-focused cognitive behavioral therapy $=$ dynamic duo of brain \& behavior change. Presented by Katrina Jefferson, MSW, LCSW, RPT-S and Patricia Grady, MSW, LICSW, RPT-S. Training attended on 9/2/2016.

National Youth Advocate Program's Trauma-focused cognitive behavioral therapy (TF-CBT). Presented by Kristine Buffington, MSW, LISW-S. Training attended 03/17/2016 through 03/18/2016. 
Professional Memberships

American Psychological Association

Graduate Student Affiliate 